\title{
Limite de escala do modelo de armadilhas numa árvore
}

\section{Renato Jacob Gava}

\author{
Tese apresentada \\ $\mathrm{aO}$ \\ Instituto de Matemática e Estatística \\ da \\ Universidade de São Paulo \\ e \\ ao \\ Centre de Mathématiques et Informatique \\ da \\ Universidade de Aix-Marseille I \\ para obtenção \\ do \\ título de Doutor em Ciências \\ Programa: Estatística \\ Orientador: Prof. Dr. Luiz Renato Fontes \\ Co-orientadora: Prof. Dr. Véronique Gayrard
}

São Paulo, outubro de 2011.

O autor agradece à FAPESP pelo apoio financeiro (Processo N. 2008/00999-0). 


\title{
Limite de escala do modelo de armadilhas numa árvore
}

\author{
Este exemplar corresponde à redação \\ final da tese devidamente corrigida \\ e defendida por Renato Jacob Gava \\ e aprovada pela Comissão Julgadora.
}

São Paulo, outubro de 2011.

\author{
Banca examinadora: \\ Prof. Dr. Luiz Renato Fontes (IME-USP) \\ Prof. Dr. Véronique Gayrard (CNRS - Université de Provence) \\ Prof. Dr. Enrique Andjel (Université de Provence) \\ Prof. Dr. Glauco Valle (IM-UFRJ) \\ Prof. Dr. Milton Jara (IMPA)
}


Para Marlene, Reinaldo e Débora 


\section{Agradecimentos}

Sou muito grato a todos com quem convivi durante todo o período de pós-graduação, desde os colegas de classe e pesquisa aos funcionários do IME. Agradeço especialmente a meu orientador Prof. Dr. Luiz Renato pela dedicação, confiança e profissionalismo; além do grande matemático conheci também o educador. A minha co-orientadora Prof. Dr. Véronique Gayrard, da Université de Provence, em Marselha, onde pude passar uma tem-

porada de valiosos estudos graças a seu apoio e orientação acadêmicos. Ao Prof. Dr. Fábio Prates, sempre solícito, pela amizade e pelos conselhos. 


\section{Resumo}

Nós apresentamos o processo K numa árvore, que é um processo de Markov com estados instantâneos e generaliza o processo $\mathrm{K}$ no grafo completo, como o limite do modelo de armadilha numa árvore, e aplicamos esse resultado para derivar um limite de escala para o modelo de armadilha do GREM.

Palavras chave: modelos de armadilhas, processo K, limite de escala, GREM. 


\section{Abstract}

We present the $\mathrm{K}$ process on a tree, which is a Markov process with instantaneous states and generalises the $\mathrm{K}$ process on the complete graph, as the limit of the trap model on a tree, and apply this result to derive a scaling limit to the GREM-like trap model.

Key words: trap model, K process, scaling limit, GREM. 


\section{Sumário}

1 Introdução $\quad 2$

2 O modelo $\quad 4$

3 Uma representação do modelo de armadilhas com $k$ níveis $\quad 8$

$4 \mathrm{O}$ processo K numa árvore $\quad 15$

5 Convergência: $\quad 23$

5.1 Resultados . . . . . . . . . . . . . . . . . . . 23

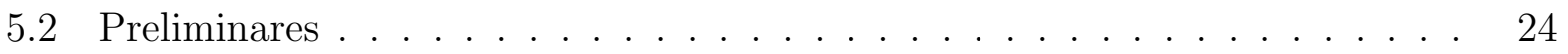

5.3 Prova do Teorema $5.1 \ldots \ldots \ldots \ldots$. . . . . . . . . . . . . . . . . . .

5.4 Medida invariante . . . . . . . . . . . . . . . . . . . 38

6 Aplicação ao modelo de armadilhas do GREM 43

6.1 Modelo de armadilhas do GREM . . . . . . . . . . . . . . . . . . . 43

6.2 Processo $\mathrm{K}$ em $\mathbb{N}_{*}^{k}$ em ambiente aleatório . . . . . . . . . . . . . . 44

6.2.1 Cascata de Poisson . . . . . . . . . . . . . . . 44

6.2.2 Processo $\mathrm{K}$ em $\mathbb{N}_{*}^{k}$ com parâmetros aleatórios . . . . . . . . . . 45

6.3 Convergência fraca de processos pontuais . . . . . . . . . . . . . . . 46

6.4 Verificação das condições $(5.2-5.5) \ldots \ldots$. . . . . . . . . . . . . . . 47

6.5 Limite de escala do modelo de armadilhas do GREM . . . . . . . . . . . . 59

$\begin{array}{ll}\text { A A métrica de Skorohod } & 60\end{array}$

B Processos Pontuais $\quad 62$

C Variação Regular $\quad 66$

$\begin{array}{ll}\text { D Variáveis aleatórias estáveis } & 67\end{array}$ 


\section{Introdução}

Esta tese aborda o modelo de armadilhas numa árvore. Grosso modo, um modelo de armadilhas é um passeio aleatório a tempo contínuo num grafo $G=(V, E)$ em ambiente aleatório, onde $G$ é não-orientado e conexo, e pode ser finito ou infinito. As armadilhas são dadas por variáveis aleatórias i.i.d. na bacia de atração de uma lei $\alpha$-estável, $0<\alpha<1$, isto é, a cada sítio $x \in V$ associamos uma variável aleatória positiva $\tau(x)$ que representa a profundidade da armadilha em $x$, e as taxas de salto entre dois sítios $x, y$ dependem de algum mecanismo relacionado a $E$ e ao ambiente. Modelos de armadilhas são propostos como protótipos para estudar o comportamento de dinâmicas em tempos grandes de sistemas desordenados; surgiram primeiramente em [5] como um modelo inicial bom para o Random Energy Model (REM), um modelo de vidro de spin introduzido em [7]. O artigo de revisão [3] e suas referências nos dão uma ideia de quanta atenção tem sido devotada na literatura matemática a esse assunto nos últimos anos.

[12] trata do modelo de armadilhas no grafo completo a baixa temperatura, também conhecido como modelo de armadilhas do REM, e seu limite de escala é obtido. O processo limite é chamado de processo K e é um processo de Markov num espaço de estados enumerável, cujos elementos são estáveis, com a exceção de um único estado, a partir do qual o processo entra em conjuntos finitos de estados estáveis com distribuição uniforme. No contexto de vidros de spin, os estados estáveis representam as configurações de baixa energia, já o estado instável representa as configurações de alta energia. Resultados de envelhecimento são obtidos. O envelhecimento é um fenômeno que tem sido intensamente estudado por físicos e, apenas recentemente, tem estado na agenda dos matemáticos. Ele ocorre longe do equilíbrio, e de modo geral, um sistema desordenado apresenta envelhecimento se existe o limite de uma função de correlação dependente de dois tempos, quando ambos os tempos divergem mantendo uma razão fixa entre eles. Grande parte dos resultados de envelhecimento foram obtidos em conexão com os modelos de armadilha, e [4], [14], [13] são alguns exemplos dessa ligação.

Nesta tese estudamos o processo K numa árvore, um modelo que generaliza o processo K no grafo completo apresentado em [12], e sua relação com o limite de escala do modelo de armadilhas correspondente ao Generalised Random Energy Model (GREM) [8] a baixa temperatura, o modelo de armadilhas do GREM. Como o próprio nome diz, o GREM é uma generalização do REM que leva em conta as correlações entre os diferentes níveis de energia, o 
que não ocorre com o REM, de forma hierárquica, com uma estrutura de árvore. O interesse no regime de baixa temperatura em ambos REM e GREM vem do fato de que, neste caso, a medida de Gibbs é essencialmente concentrada em um pequeno número de níveis de energia, ou seja, ela dá massa às configurações de spin de valores extremos mínimos, ao passo que no de alta temperatura a distribuição de Gibbs é concentrada em um número de níveis de energia que cresce com $n$ quando $n$ tende ao infinito. A fim de ampliar o trabalho desta tese, o seu passo seguinte deve ser o entendimento do fenômeno de envelhecimento relacionado ao processo de K numa árvore.

O texto está dividido da seguinte maneira. No capítulo 2 apresentamos o modelo de armadilhas numa árvore e o chamamos de $Z_{k}^{F}$. No capítulo 3 construímos um processo $X_{k}^{F}$ que, sob uma escolha específica de parâmetros, tem a mesma distribuição de $Z_{k}^{F}$. No capítulo 4 nós definimos o processo em volume infinito $X_{k}$, chamado o processo $\mathrm{K}$ numa árvore. No capítulo 5 permitimos que todos os parâmetros do processo em volume finito dependam de $n$, ou seja, mudamos o sobrescrito " $F$ " por " $(n)$ " para enfatizar a dependência em $n$ e então consideramos o processo $X_{k}^{(n)}$ do capítulo 3. Então, sob determinadas condições assintóticas desses parâmetros quando $n \rightarrow \infty$, mostramos que a distribuição de $X_{k}^{(n)}$ converge para o de $X_{k}$.

Finalmente, no capítulo 6, consideramos um caso particular de $X_{k}^{F}$, onde o parâmetros são definidos em termos de variáveis aleatórias com cauda pesada. Então, tomando uma escala adequada do processo, que inclui uma relação de escala particular entre os volumes $M_{1}, \ldots, M_{k}$, estabelecemos sua convergência graças à redução deste caso à situação do capítulo 5 e à verificação da condições de seu teorema da convergência. 


\section{O modelo}

Primeiramente descrevemos o modelo de armadilhas numa árvore. Comecemos com a árvore. Ao longo do texto $k$ será um inteiro fixo em $\mathbb{N}_{*}:=\{1,2, \ldots\}$. Considere $k$ números $M_{1}, \ldots, M_{k} \in \mathbb{N}_{*}$, algumas vezes chamados volumes, e defina $\mathcal{M}_{j}=\left\{1, \ldots, M_{j}\right\}, j=$ $1, \ldots, k$. Consideremos então a árvore $\mathbb{T}_{k}^{F}$ de raiz $\emptyset$, cuja primeira geração é dada por $\mathcal{M}_{1}$, e tal que, dado um vértice $\left.x\right|_{i}$ da $i$-ésima geração de $\mathbb{T}_{k}^{F}, 1 \leq i<k$, o conjunto de descendentes de $\left.x\right|_{i}$, pertencendo à $i+1$-ésima geração de $\mathbb{T}_{k}^{F}$, é dado por $\left\{\left.x\right|_{i}\right\} \times \mathcal{M}_{i+1}$. $\mathrm{O}$ conjunto de vértices de $\mathbb{T}_{k}^{F}$ pode assim ser representado como se segue

$$
V_{k}^{F}=\left\{\emptyset,\left.x\right|_{i} \equiv x_{1} \ldots x_{i}: x_{j} \in \mathcal{M}_{j}, 1 \leq j \leq i \leq k\right\}
$$

Usaremos também a notação

$$
\left.x\right|^{i}=x_{i} \ldots x_{k},\left.\quad \mathcal{M}\right|_{i}=\mathcal{M}_{1} \times \ldots \times \mathcal{M}_{i},\left.\quad \mathcal{M}\right|^{i}=\mathcal{M}_{i} \times \ldots \times \mathcal{M}_{k},
$$

$1 \leq i \leq k$. Abaixo identificaremos $\left.x\right|_{i}$ and $\left.x\right|^{i}$ com vetores (com respectivamente $i$ e $k-i+1$ dimensões).

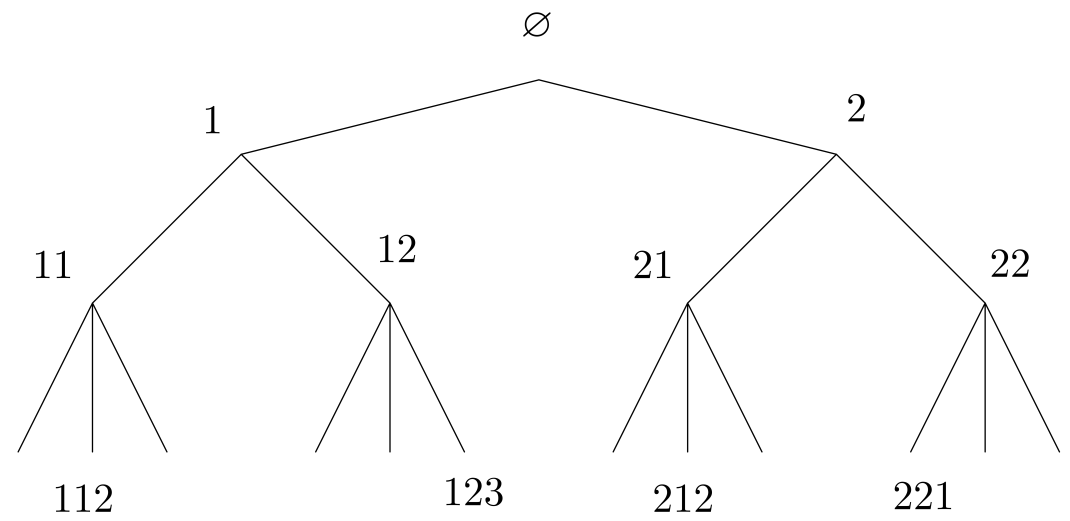

Figura 1: $k=3, M_{1}=M_{2}=2, M_{3}=3$.

$\mathbb{T}_{k}^{F}$ é claramente uma árvore finita e isso é enfatizado na notação pelo uso do sobrescrito "F". Mais tarde definiremos um processo análogo para uma árvore infinita (com $k$ gerações), e neste caso não haverá sobrescrito. Entendemos a raiz como a 0-ésima geração de $\mathbb{T}_{k}^{F}$, e $\left.\left.x\right|_{i} \in \mathcal{M}\right|_{i}$ como um elemento de sua $i$-ésima geração, $1 \leq i \leq k$. Por vezes usaremos simplesmente $x$ em vez de $\left.x\right|_{k}$. Nos capítulos abaixo, consideraremos a árvore inifinita, 
denotada por $\mathbb{T}_{k}$, com conjunto de vértices

$$
V_{k}=\left\{\emptyset,\left.x\right|_{i} \equiv x_{1} \ldots x_{i}: x_{j} \in \mathbb{N}_{*}, 1 \leq j \leq i \leq k\right\}
$$

e estrutura de gerações paralela a $\mathbb{T}_{k}^{F}$ de modo óbvio, com notação paralela, sem o " $F$ " sobrescrito, para objetos paralelos.

Nosso processo é uma processo de Markov a tempo contínuo cujo espaço de estados é o conjunto de folhas de $\mathbb{T}_{k}^{F}$, ou em outras palavras, o conjunto de vértices da $k$-ésima geração de $\mathbb{T}_{k}^{F}$, a saber

$$
L_{k}^{F}=\left\{\left.x\right|_{k}:\left.x\right|_{k} \in V_{k}^{F}\right\}
$$

Da identificação em (2.1), é claro que $L_{k}^{F}$ pode por sua vez ser identificado com $\left.\mathcal{M}\right|_{k}$.

Descrevemos agora o mecanismo de transição de nosso processo. Haverá um conjunto de parâmetros para isso. A fim de diferenciar a descrição da árvore finita da posterior árvore infinita, por um lado, e para enfatizar a analogia entre os dois casos, por outro lado, continuamos a recorrer ao uso do sobrescrito " $F$ " também para o conjunto de parâmetros do processo em volume finito.

Para $j=1, \ldots, k-1$, sejam

$$
p_{j}^{F}:\left.\mathcal{M}\right|_{j} \rightarrow(0,1)
$$

e

$$
\gamma_{k}^{F}:\left.\mathcal{M}\right|_{k} \rightarrow(0, \infty)
$$

Para $\left.x \in \mathcal{M}\right|_{k}$, seja $g_{x} \in\{0,1, \ldots, k-1\}$ uma variável aleatória definida num espaço de probabilidade $\left(\Omega^{\prime}, \mathcal{A}, \mathbb{P}\right)$ tal que

$$
\mathbb{P}\left(g_{x}=i\right)=\left[1-p_{i}^{F}\left(\left.x\right|_{i}\right)\right] \prod_{j=i+1}^{k-1} p_{j}^{F}\left(\left.x\right|_{j}\right)
$$

onde por convenção $\Pi_{j=k}^{k-1} p_{j}^{F}\left(\left.x\right|_{i}\right)=1$ and $p_{0}^{F} \equiv 0$.

Seja $Z_{k}^{F}$ uma cadeia de Markov a tempo contínuo em $\left.\mathcal{M}\right|_{k}$ como se segue. Quando $Z_{k}^{F}$ está em $\left.x \in \mathcal{M}\right|_{k}$ espera um tempo exponencial de média $\gamma_{k}^{F}(x)$ e então salta da seguinte maneira. Ele primeiramente olha para uma cópia $g_{x}^{\prime}$ de $g_{x}$ (independente a cada vez das outras olhadas anteriores). Se $g_{x}^{\prime}=j$, então, denotando por $a_{x}(j)$ o único ancestral de $x$ na geração $j$ de $\mathbb{T}_{k}^{F}$ (a saber $a_{x}(j)=\left.x\right|_{j}$ ), $Z_{k}^{F}$ salta com lei uniforme para um dos descendentes de $a_{x}(j)$ em $\left.\mathcal{M}\right|_{k}$. Em outras palavras, dado que $g_{x}^{\prime}=j$, então as coordenadas $\left.x\right|_{j}\left(=a_{x}(j)\right)$ de $x$ são mantidas pelo salto, e as demais coordenadas são escolhidas uniformemente em 


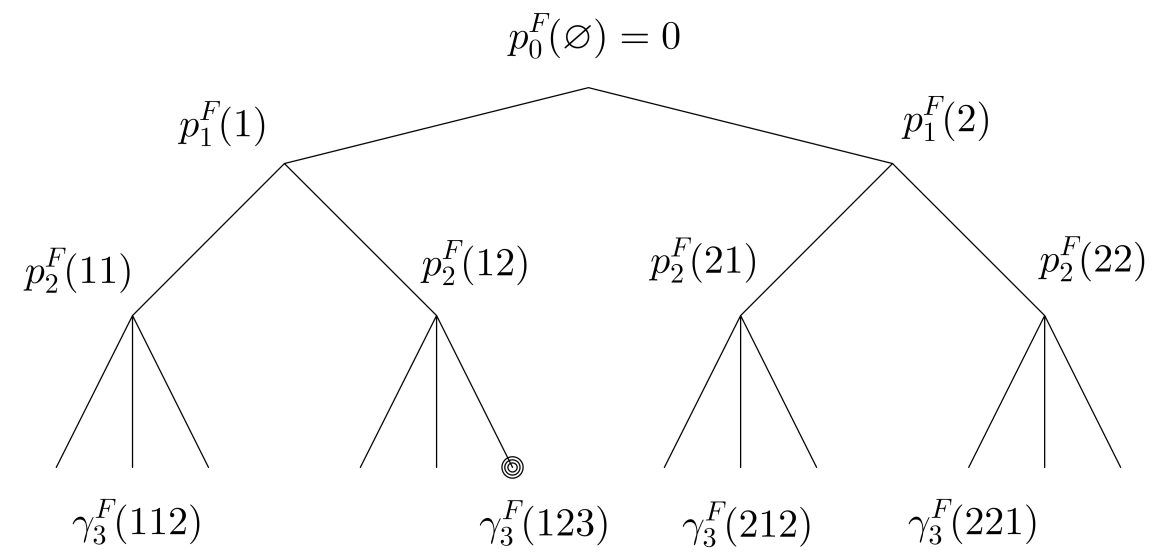

Figura 2: $k=3, M_{1}=M_{2}=2, M_{3}=3$.

$\left.\mathcal{M}\right|^{j+1}$. Podemos então dizer que a distribuição de transição da cadeia de Markov a tempo discreto imersa de $Z_{k}^{F}$ que parte de $x$ é a distribuição sobre os descendentes de um ancestral de $x$ cuja geração é aleatoriamente escolhida conforme a distribuição de $g_{x}$.

Observação 2.1. Podemos entender a variável aleatória $g_{x}$ como se segue. Anexemos moedas aos sítios da árvore que não são folhas, ou seja, $\left.\mathcal{M}\right|_{k-1}$, de tal forma que a probabilidade de cara da moeda no sítio $\left.\left.x\right|_{j} \in \mathcal{M}\right|_{j}$ seja $p_{j}^{F}\left(\left.x\right|_{j}\right), j=1, \ldots, k-1$. A moeda da raiz tem probabilidade $p_{0}^{F}=0$ de dar cara. Quando decide saltar do sítio $\left.x \in \mathcal{M}\right|_{k}$, em primeiro lugar $Z_{k}^{F}$ lança sucessivamente as moedas de $\left.x\right|_{k-1}, \ldots,\left.x\right|_{1}, \emptyset$ (nessa ordem) até obter coroa pela primeira vez, e então ele pára no respectivo sítio. Observe que esse procedimento está quase certamente bem definido já que $p_{0}^{F}=0$. Dado que $\left.x\right|_{j}, j=0, \ldots, k-1$ (onde $\left.x\right|_{0} \equiv \emptyset$ ), foi o sítio de parada do procedimento, então $g_{x}=j$.

Definição 2.1. Chamamos $Z_{k}^{F}$ um modelo de armadilhas em $\mathbb{T}_{k}^{F}$, ou modelo de armadilhas com $k$ níveis, com parâmetro de tempo de espera $\gamma_{k}^{F}$ e parâmetros de ativação $\left(p_{j}^{F}\right)_{j=1}^{k-1}$.

Notação: $Z_{k}^{F} \sim T M_{k}\left(\mathbb{T}_{k}^{F} ; \gamma_{k}^{F} ;\left(p_{j}^{F}\right)_{j=1}^{k-1}\right)$.

Observação 2.2. A cadeia de Markov $Z_{k}^{F}$ é reversível e sua medida invariante é dada por

$$
\mathcal{G}_{k}^{F}(y)=\frac{\gamma_{k}^{F}(y)\left[\prod_{i=1}^{k-1} p\left(\left.y\right|_{i}\right)\right]^{-1}}{\sum_{\left.x \in \mathcal{M}\right|_{k}} \gamma_{k}^{F}(x)\left[\prod_{i=1}^{k-1} p\left(\left.x\right|_{i}\right)\right]^{-1}} .
$$

A fim de tornar claro (2.8), detalhemos as taxas de transição de $Z_{k}^{F}$. Dados $x,\left.y \in \mathcal{M}\right|_{k}$ $(x \neq y)$, ponha $m=\min _{i=1, \ldots, k-1}\left\{i ; x_{i} \neq y_{i}\right\}$ e $m=\min \{\emptyset\}=k$. Se denotarmos por $Q$ a matriz 
de taxa de transição e por $q(\cdot, \cdot)$ seus elementos, então

$$
q(x, y)=\frac{1}{\gamma_{k}^{F}(x) M_{k}} \sum_{i=0}^{m-1}\left[1-p_{i}^{F}\left(\left.x\right|_{i}\right)\right] \prod_{j=i+1}^{k-1} \frac{p_{j}^{F}\left(\left.x\right|_{j}\right)}{M_{j}}
$$

é a taxa de transição do estado $x$ para o estado $y$, e só nos resta verificar a equação de balanceamento.

Estamos interessados em tomar os limites de $Z_{k}^{F}$ quando $M_{1}, \ldots, M_{k} \rightarrow \infty$ (sob condições apropriadas sobre seus parâmetros). Para isso, será conveniente considerar uma construção particular desse processo. No próximo capítulo nós o faremos. Defineremos um processo $X_{k}^{F}$ em $V_{k}^{F}$ de um modo particular, com certos parâmetros, e no fim demonstraremos que com a relação correta entre esses parâmetros e os de $Z_{k}^{F}$, obtemos que $X_{k}^{F}$ e $Z_{k}^{F}$ têm a mesma distribuição. 


\section{Uma representação do modelo de armadilhas com $k$ níveis}

$\operatorname{Sejam} \mathbb{N}_{*}=\{1,2, \ldots\}, \overline{\mathbb{N}}_{*}=\mathbb{N}_{*} \cup\{\infty\}$ e $\mathbb{R}_{+}:=[0, \infty)$. Dados $M_{1}, \ldots, M_{k} \in \mathbb{N}_{*}$, e recordando a notação do Capítulo 2 acima, sejam $\gamma_{j}^{F}:\left.\mathcal{M}\right|_{j} \rightarrow(0, \infty), j=1, \ldots, k$, e defina

$$
\bar{\gamma}_{j}^{F}\left(\left.x\right|_{j}\right):=\gamma_{1}^{F}\left(\left.x\right|_{1}\right) \times \gamma_{2}^{F}\left(\left.x\right|_{2}\right) \times \ldots \times \gamma_{j}^{F}\left(\left.x\right|_{j}\right)
$$

Abaixo identificaremos $\bar{\gamma}_{j}^{F}$ com uma medida em $\left.\mathcal{M}\right|_{j}$ para $j=1, \ldots, k$, com $\bar{\gamma}_{j}^{F}(\cdot)$ fazendo o papel de $\bar{\gamma}_{j}^{F}(\{\cdot\})$.

Neste capítulo construíremos um processo $X_{k}^{F}$ em $\left.\mathcal{M}\right|_{k}$ de um modo indutivo e consideraremos todas as variáveis aleatórias subsequentes definidas num espaço de probabilidade $\left(\Omega^{\prime}, \mathcal{A}, \mathbb{P}\right)$. Primeiramente definimos o processo $X_{1}^{F}$. Ele é uma cadeia de Markov a tempo contínuo em $\left.\mathcal{M}\right|_{1}$ que quando está em $\left.x_{1} \in \mathcal{M}\right|_{1}$ espera um tempo exponencial de média $\gamma_{1}^{F}\left(x_{1}\right)$ e então salta uniformemente para um sítio em $\left.\mathcal{M}\right|_{1}$. Construíremos $X_{1}^{F}$ da seguinte maneira.

Sejam $\mathcal{N}_{1}=\left\{\left(N_{r}^{\left(x_{1}, 1\right)}\right)_{r \geq 0}, x_{1} \in \mathbb{N}_{*}\right\}$ i.i.d. processos de Poisson de taxa 1, e seja $\sigma_{i}^{x_{1}, 1}$ a $i$-ésima ocorrência de $N^{\left(x_{1}, 1\right)}, i \geq 1$. Chamaremos $\mathcal{S}_{1}^{F}=\left\{\sigma_{i}^{\left(x_{1}, 1\right)} ; x_{1} \in \mathcal{M}_{1}, i \geq 1\right\}$ o conjunto de marcas do primeiro nível de $X_{k}^{F}$. Sejam $\mathcal{T}_{1}=\left\{T_{s}^{(1)}, s \in \mathbb{R}_{+}\right\}$variáveis aleatórias i.i.d. exponenciais de taxa 1 . Considere $\mathcal{N}_{1}$ e $\mathcal{T}_{1}$ independentes.

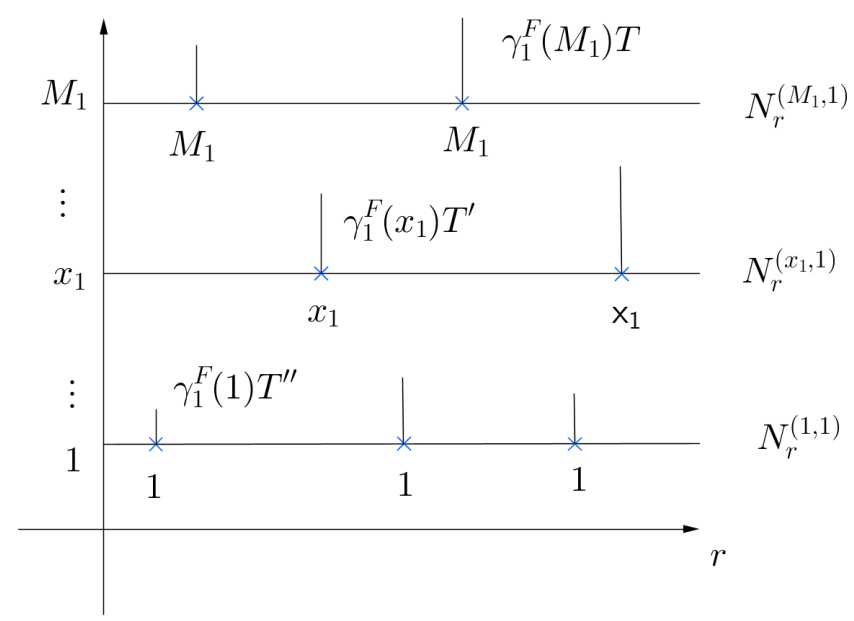

Figura 3: Marcas de Poisson para $k=1$ e $M_{1}$ qualquer. 
Para $s \in \mathcal{S}_{1}^{F}$, seja

$$
\xi_{1}^{F}(s)=x_{1} \text { se } s=\sigma_{j}^{x_{1}, 1} \text { para algum } x_{1} \in \mathcal{M}_{1} \text { e } j \geq 1 .
$$

Note que $\xi_{1}^{F}$ está bem definido quase certamente, ou seja, para $s_{1}, s_{2} \in \mathcal{S}_{1}^{F}$ temos $s_{1} \neq s_{2}$ quase certamente. Agora definamos uma medida aleatória $\mu_{1}^{F}$ em $\mathbb{R}_{+}$como se segue:

$$
\mu_{1}^{F}(\{s\})=\left\{\begin{array}{l}
\gamma_{1}^{F}\left(\xi_{1}^{F}(s)\right) T_{s}^{(1)}, \text { se } s \in \mathcal{S}_{1}^{F} \\
0, \text { se } s \in \mathbb{R}_{+} \backslash \mathcal{S}_{1}^{F} .
\end{array}\right.
$$

Observação 3.1. Notamos que $\xi_{1}^{F}(s), s \in \mathcal{S}_{1}^{F}$, são uniformes i.i.d. em $\mathcal{M}_{1}$.

Para $r \geq 0$, seja

$$
\Gamma_{1}^{F}(r):=\mu_{1}^{F}([0, r])=\sum_{x_{1}=1}^{M_{1}} \sum_{s \in\left\{\sigma_{i}^{\left(x_{1}, 1\right)} ; i \geq 1\right\} \cap[0, r]} \gamma_{1}^{F}\left(x_{1}\right) T_{s}^{(1)} .
$$

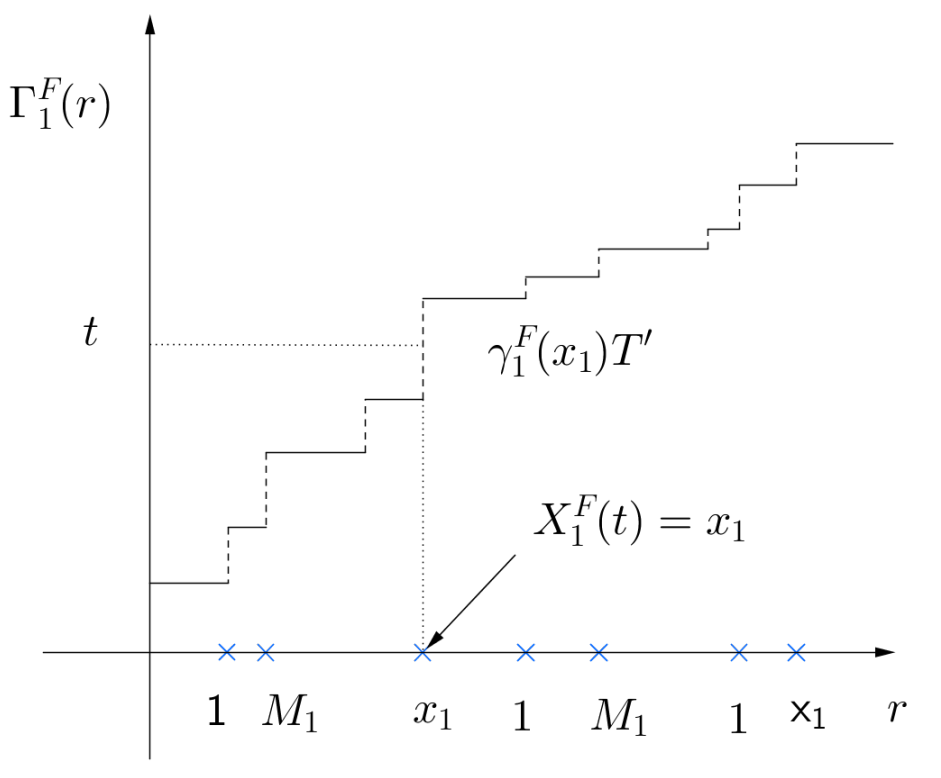

Figura 4: Uma realização de $X_{1}^{F}$.

Para $t \geq 0$, seja

$$
\varphi_{1}^{F}(t):=\left(\Gamma_{1}^{F}\right)^{-1}(t)=\inf \left\{r \geq 0 ; \Gamma_{1}^{F}(r)>t\right\}
$$

a inversa contínua à direita de $\Gamma_{1}^{F}$. 
Definimos o processo $\left(X_{1}^{F}, Y_{1}^{F}\right)$ em $\left(\left.\mathcal{M}\right|_{1}, \mathbb{R}_{+}\right)$como se segue. Para $t \geq 0$

$$
\left(X_{1}^{F}, Y_{1}^{F}\right)(t)=\left(\xi_{1}^{F}\left(\varphi_{1}^{F}(t)\right), \varphi_{1}^{F}(t)\right) .
$$

Suponhamos que $\left(X_{j}^{F}, Y_{j}^{F}\right)$ esteja definido para $j=1, \ldots, \ell-1, \ell \leq k$.

Definição 3.1. Um intervalo $I \subset \mathbb{R}_{+}$é um intervalo de constância de $\left(X_{j}^{F}, Y_{j}^{F}\right)$ se $\left(X_{j}^{F}, Y_{j}^{F}\right)$ for constante sobre I, ou seja,

$$
\left(X_{j}^{F}, Y_{j}^{F}\right)(r)=\left(X_{j}^{F}, Y_{j}^{F}\right)(s) \text { para todo } r, s \in I,
$$

e I for maximal com essa propriedade.

A condição de maximalidade e a continuiudade à direita de $\left(X_{j}^{F}, Y_{j}^{F}\right)$ implicam que $I=[a, b)$ para algum $0 \leq a<b$. Agora estamos prontos para definir $\left(X_{\ell}^{F}, Y_{\ell}^{F}\right)$ para $2 \leq \ell \leq k$.

Seja $\mathcal{I}_{\ell-1}^{F}$ a coleção de intervalos de constância de $\left(X_{\ell-1}^{F}, Y_{\ell-1}^{F}\right)$. Sejam $\mathcal{N}_{\ell}=\left\{\left(N_{r}^{\left(x_{\ell}, \ell\right)}\right)_{r \geq 0}\right.$, $\left.x_{\ell} \in \mathbb{N}_{*}\right\}$ i.i.d. processos de Poisson de taxa 1. Considere $\sigma_{i}^{\left(x_{\ell}, \ell\right)}$ a $i$-ésima ocorrência de $N^{\left(x_{\ell}, \ell\right)}, i \geq 1$. Chamaremos $\mathcal{S}_{\ell}^{F}=\left\{\sigma_{i}^{\left(x_{\ell}, \ell\right)} ; x_{\ell} \in \mathcal{M}_{\ell}, i \geq 1\right\}$ o conjunto de marcas de Poisson do $\ell$-ésimo nível, e $\mathcal{R}_{\ell}^{F}=\left\{b ; I=[a, b)\right.$ e $\left.I \in \mathcal{I}_{\ell-1}^{F}\right\}$ o conjunto de marcas extras do $\ell$-ésimo nível. Note que $\mathcal{R}_{\ell}^{F}$ é o conjunto de extremidades à direita de intervalos de $\mathcal{I}_{\ell-1}$. Chamamos $\mathcal{S}_{\ell}^{F} \cup \mathcal{R}_{\ell}^{F}$ o conjuntos de marcas do $\ell$-ésimo nível. Sejam $\mathcal{T}_{\ell}=\left\{T_{s}^{(\ell)}, s \in \mathbb{R}_{+}\right\}$variáveis aleatórias i.i.d. exponenciais de média 1. Assumimos $\mathcal{N}_{\ell}$ e $\mathcal{T}_{\ell}$ independentes e também independentes de $\mathcal{N}_{j}$ e $\mathcal{T}_{j}$ para $j<\ell$.

Para cada $s \in \mathcal{R}_{\ell}^{F}$, associamos uma variável aleatória uniforme $U_{\ell}(s)$ em $\left\{1, \ldots, M_{\ell}\right\}$. Assuma que $\left\{U_{\ell}(s), s \in \mathcal{R}_{\ell}^{F}, \ell \geq 1\right\}$ são mutualmente independentes e independentes das outras variáveis aleatórias do modelo. Seja

$$
\xi_{\ell}^{F}(s)=\left\{\begin{array}{cl}
x_{\ell}, & \text { se } s=\sigma_{j}^{\left(x_{\ell}, \ell\right)} \text { para algum } x_{\ell} \in \mathcal{M}_{\ell} \text { e } \ell \geq 1, \\
U_{\ell}(s), & \text { se } s \in \mathcal{R}_{\ell}^{F} .
\end{array}\right.
$$

Chamaremos $\xi_{\ell}^{F}(s)$ o rótulo de $s \in \mathcal{R}_{\ell}^{F} \cup \mathcal{S}_{\ell}^{F}, 1 \leq \ell \leq k$ (onde $\mathcal{R}_{1}^{F}=\emptyset$ ).

Note que no primeiro caso acima $s \in \mathcal{S}_{\ell}^{F}$, e que $\xi_{\ell}^{F}$ está bem definida quase certamente. Definamos agora uma medida $\mu_{\ell}^{F}$ em $\mathbb{R}_{+}$como se segue:

$$
\mu_{\ell}^{F}(\{s\})=\left\{\begin{array}{l}
\gamma_{\ell}^{F}\left(X_{\ell-1}^{F}(s), \xi_{\ell}^{F}(s)\right) T_{s}^{(\ell)}, \text { se } s \in \mathcal{S}_{\ell}^{F} \\
\gamma_{\ell}^{F}\left(X_{\ell-1}^{F}(s-), \xi_{\ell}^{F}(s)\right) T_{s}^{(\ell)}, \text { se } s \in \mathcal{R}_{\ell}^{F}
\end{array}\right.
$$

e $\mu_{\ell}^{F}\left(\mathbb{R}_{+} \backslash\left(\mathcal{S}_{\ell}^{F} \cup \mathcal{R}_{\ell}^{F}\right)\right)=0$. Note que $\mathcal{S}_{\ell}^{F} \cap \mathcal{R}_{\ell}^{F}(\omega)=\emptyset$ para quase toda realização $\omega \in \Omega^{\prime}$. 


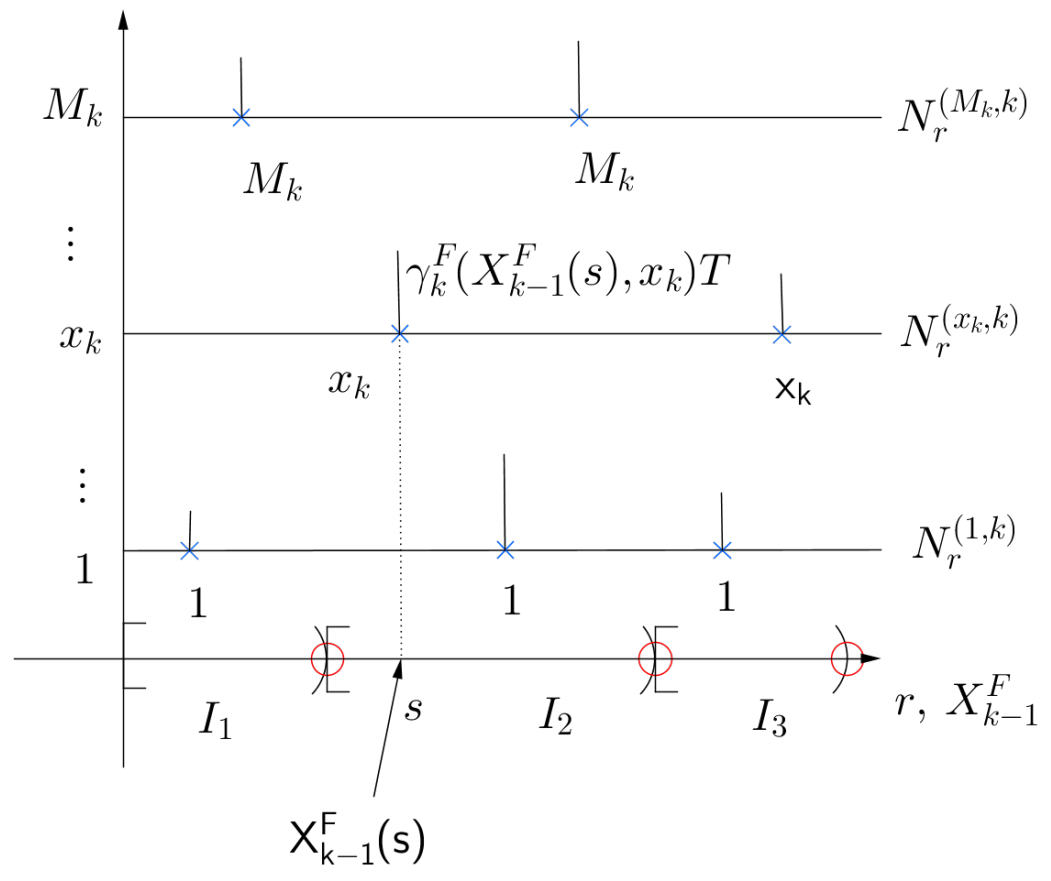

Figura 5: Marcas de Poisson e extras para $k>1$ e $M_{k}$ qualquer.

Observação 3.2. Ressaltamos que $\xi_{j}^{F}(s), s \in \mathcal{S}_{j}^{F}$, são uniformes i.i.d. em $\mathcal{M}_{j}, j=1, \ldots, \ell$.

Para $r \geq 0$, seja

$$
\Gamma_{\ell}^{F}(r):=\mu_{\ell}^{F}([0, r])
$$

Para $t \geq 0$, seja

$$
\varphi_{\ell}^{F}(t):=\left(\Gamma_{\ell}^{F}\right)^{-1}(t)=\inf \left\{r \geq 0: \Gamma_{\ell}^{F}(r)>t\right\}
$$

inversa contínua à direita de $\Gamma_{\ell}^{F}$.

Definimos o processo $\left(X_{\ell}^{F}, Y_{\ell}^{F}\right)$ on $\left(\left.\mathcal{M}\right|_{\ell}, \mathbb{R}_{+}^{\ell}\right)$ como se segue. Para $t \geq 0$

$$
\left(X_{\ell}^{F}, Y_{\ell}^{F}\right)(t)=\left\{\begin{array}{l}
\left(\left(X_{\ell-1}^{F}\left(\varphi_{\ell}^{F}(t)\right), \xi_{\ell}^{F}\left(\varphi_{\ell}^{F}(t)\right)\right) ;\left(Y_{\ell-1}^{F}\left(\varphi_{\ell}^{F}(t)\right), \varphi_{\ell}^{F}(t)\right)\right) \text { se } \varphi_{\ell}^{F}(t) \in \mathcal{S}_{\ell}^{F} \\
\left(\left(X_{\ell-1}^{F}\left(\varphi_{\ell}^{F}(t)-\right), \xi_{\ell}^{F}\left(\varphi_{\ell}^{F}(t)\right)\right) ;\left(Y_{\ell-1}^{F}\left(\varphi_{\ell}^{F}(t)\right), \varphi_{\ell}^{F}(t)\right)\right) \text { se } \varphi_{\ell}^{F}(t) \in \mathcal{R}_{\ell}^{F}
\end{array}\right.
$$

Observação 3.3. Notamos que cada intervalo $I$ de $\mathcal{I}_{j}^{F}, j \geq 2$, pode ser identificado com um salto de $\Gamma_{j}^{F}$, isto é, $\mathcal{I}_{j}^{F}=\left\{\left[\Gamma_{j}^{F}(r-), \Gamma_{j}^{F}(r)\right) ; r \geq 0\right\}=\left\{I_{j}^{F}(s):=\left[\Gamma_{j}^{F}(s-), \Gamma_{j}^{F}(s)\right) ; s \in\right.$ $\left.\mathcal{S}_{j}^{F} \cup \mathcal{R}_{j}^{F}\right\}$, e $\left\{\left|I_{j}^{F}(s)\right|, s \in \mathcal{S}_{j}^{F} \cup \mathcal{R}_{j}^{F}\right\}$ são variáveis aleatórias exponenciais independentes com média

$$
\left\{\gamma_{j}^{F}\left(X_{j-1}^{F}(s), \xi_{j}^{F}(s)\right), s \in \mathcal{S}_{j}^{F} ; \gamma_{j}^{F}\left(X_{j-1}^{F}(s-), \xi_{j}^{F}(s)\right), s \in \mathcal{R}_{j}^{F}\right\}
$$




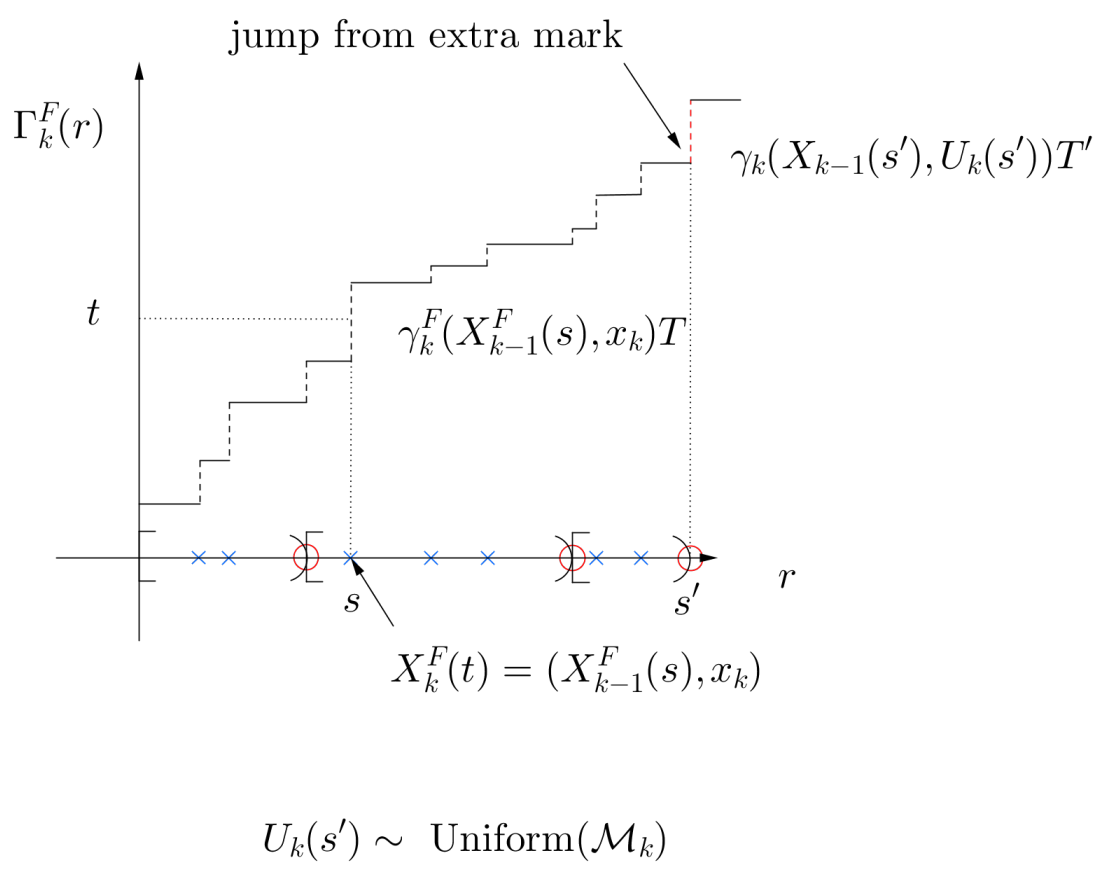

Figura 6: Uma realização de $X_{k}^{F}$.

respectivamente.

Neste ponto podemos observar que nosso interesse está em $X_{k}^{F} . Y_{k}^{F}$ é introduzido exclusivamente por nos permitir distinguir pontos $s \in \mathcal{S}_{\ell}^{F} \cup \mathcal{R}_{\ell}^{F}$ no caso de duas marcas consecutivas com os mesmos rótulos, o que como veremos no capítulo seguinte não ocorre no caso de volume infinito. De fato, no Capítulo 4 abaixo introduziremos uma versão em volume infinito de $X_{k}^{F}$ para a qual a versão respectiva de $Y_{k}^{F}$ não será necessária explicitamente, e portanto não explicitamente introduzida.

Observação 3.4. Advertimos que o número de marcas de $\mathcal{S}_{j}^{F} \cup \mathcal{R}_{j}^{F}$ em cada intervalo fechado $\bar{I}$, com $I \in \mathcal{I}_{j-1}^{F}$, é igual a $1+$ uma variável aleatória geométrica de média $M_{j} \gamma_{j-1}^{F}\left(X_{j-1}^{F}(s)\right)$, onde $s \in \mathcal{S}_{j-1}^{F} \cup \mathcal{R}_{j-1}^{F}$ é tal que $I=I_{j-1}^{F}(s)$, e cada intervalo fechado tem exatamente uma marca de $\mathcal{R}_{j}^{F}$, em sua extremidade direita.

Definição 3.2. Chamamos $X_{k}^{F}$ definido acima o modelo de armadilhas em $\mathbb{T}_{k}^{F}$, ou modelo de armadilhas com $k$ niveis, com conjunto de parâmetros $\underline{\gamma_{k}^{F}}=\left\{\gamma_{i}^{F} ; i=1, \ldots, k\right\}$.

Notação: $X_{k}^{F} \sim T M_{k}\left(\mathbb{T}_{k}^{F} ; \underline{\gamma_{k}^{F}}\right)$.

Agora fazemos a conexão entre os modelos do Capítulo 2 e Capítulo 3, o qual em particular estabelece que o último modelo é uma representação do primeiro sob a relação apropriada 
de seus respectivos conjuntos de parâmetros, assim justificando a terminologia comum. Seja $X_{k}^{F}$ como acima, e seja $Z_{k}^{F}$ como no Capítulo 2 , isto é, $Z_{k}^{F} \sim T M_{k}\left(\left.\mathcal{M}\right|_{k} ; \gamma_{k}^{F},\left(p_{j}^{F}\right)_{j=1}^{k-1}\right)$.

Lema 3.1. Suponha

$$
p_{j}^{F}\left(\left.x\right|_{j}\right):=\frac{1}{1+M_{j+1} \gamma_{j}^{F}\left(\left.x\right|_{j}\right)}
$$

para todo $\left.\left.x\right|_{j} \in \mathcal{M}\right|_{j}$ e $j=1, \ldots, k-1$. Então $X_{k}^{F}$ e $Z_{k}^{F}$ têm a mesma distribuição.

Demonstração. Antes da prova em si tornamos claro um ponto. Seja $Z_{k, i}^{F}, i=1, \ldots, k$ a $i$ ésima coordenada de $Z_{k}^{F}=\left(Z_{k, 1}^{F}, \ldots, Z_{k, k}^{F}\right)$, e seja $\left.Z_{k}^{F}\right|_{j}=\left(Z_{k, 1}^{F}, \ldots Z_{k, j}^{F}\right), j=1, \ldots, k$. Como apontado no Capítulo 2 acima, a cadeia imersa de $Z_{k}^{F}$, chamemo-la $J_{k}^{F}=\left(J_{k, 1}^{F}, \ldots, J_{k, k}^{F}\right)$, com $\left.J_{k}^{F}\right|_{j}=\left(J_{k, 1}^{F}, \ldots, J_{k, j}^{F}\right), j=1, \ldots, k$, pode ser descrita em termos de lançamentos sucessivos de moedas dos vértices $\left.J_{k}^{F}\right|_{k-1},\left.J_{k}^{F}\right|_{k-2}, \ldots,\left.J_{k}^{F}\right|_{1}$ (veja Observação 2.1). Considere o evento $A_{k-1}=\left\{\right.$ lançamento da moeda de $\left.J_{k}^{F}\right|_{k-1}$ é o primeiro a dar cara $\}$. Em termos de variáveis aleatórias $g_{J_{k}^{F}}$, temos $A_{k-1}=\left\{g_{J_{k}^{F}}<k-1\right\}$. Agora abservamos que, dado $A_{k-1}$, a distribuição do salto de $\left.J_{k}^{F}\right|_{k-1}$ é a mesma que aquela de $J_{k-1}^{F}$. Já que a distribuição de salto de $J_{k, k}^{F}$ é sempre uniforme em $\mathcal{M}_{k}$, temos que, dado $A_{k-1}$, a distribuição de salto de $J_{k}^{F}$ é a mesma que a distribuição conjunta do salto de $J_{k-1}^{F}$ e uma variável uniforme independente em $\mathcal{M}_{k}$.

Para $k=1$, observamos que as probabilidades de transição de $X_{1}^{F}$ são sempre uniformes em $\mathcal{M}_{1}$, já que os rótulos dos sucessivos pontos de $\mathcal{S}_{1}^{F}$ têm essa propriedade e são independentes ums dos outros. Assim, $X_{1}^{F}$ e $Z_{1}^{F}$ são processos com o mesmo espaço de estados e probabilidades de transição, e os tempos de espera claramente podem ser casados. O resultado se segue para $k=1$.

Agora procedemos por indução sobre $k$. Suponhamos que o resultado valha para $k=K-$ 1 para algum $K \geq 2$. Para mostrar que $X_{K}^{F} \sim Z_{K}^{F}$, é suficiente identificar os mecanismos de transição de ambos os processos. Fixemos um tempo $t \geq 0$ e um ponto $\left.\left.x\right|_{K} \in \mathcal{M}\right|_{K}$. Dado que ambos processos estão em $\left.x\right|_{K}$ no tempo $t$, então ambos tempos de salto são exponencialmente distribuídos com média $\gamma_{K}^{F}\left(\left.x\right|_{K}\right)$. Até aqui, temos uma identificação. Agora identifiquemos os mecanismos de salto dos dois processos.

Como discutido no primeiro parágrafo acima, dado $A_{K-1}$ temos que $\left.J_{K}^{F}\right|_{K-1}$ salta como $J_{K-1}^{F}$, e $J_{K, K}^{F}$ salta uniformemente em $\mathcal{M}_{K}$, e os saltos de $\left.J_{K}^{F}\right|_{K-1}$ e $J_{K, K}^{F}$ são independentes. Identifiquemos um mecanismo de lançmento de moedas no salto de $X_{K}^{F}$. Para isso, definamos para $I \in \mathcal{I}_{K-1}^{F}$ a variável $N_{I}$ que conta o número de marcar de $\mathcal{S}_{K}^{F} \cup \mathcal{R}_{K}^{F}$ no fecho $\bar{I}$ de $I$, 
ou seja, $N_{I}=\left|\bar{I} \cap\left(\mathcal{S}_{K}^{F} \cup \mathcal{R}_{K}^{F}\right)\right|$. Relembramos da Observação 3.4 que $N_{I}$ iguala-se a $1+$ uma variável aleatória geométrica de média $M_{K} \gamma_{K-1}^{F}\left(X_{K-1}^{F}(s)\right)$, onde $s \in I$ (recorde que $X_{K-1}^{F}$ é constante em $I)$.

O mecanismo no processo $X_{K}^{F}$ que desempenha o papel do primeiro lançamento de moeda é se ou não $\varphi_{K}(t)$, o ponto de $\mathcal{S}_{K}^{F} \cup \mathcal{R}_{K}^{F}$ no intervalo de constância corrente de $X_{K-1}^{F}$, é um ponto de $\mathcal{S}_{K}^{F}$ ou de $\mathcal{R}_{K}^{F}$. Considere o último caso o evento $\bar{A}_{K-1}=\left\{\varphi_{K}(t) \in \mathcal{R}_{K}^{F}\right\}$, o qual corresponde à cara, e significa que $\left.X_{K}^{F}\right|_{K-1}$ fará um salto, que identificamos como um salto de $X_{K-1}^{F}$, independente do (uniforme em $\mathcal{M}_{K}$ ) salto acompanhante de $X_{K, K}^{F}$.

Neste ponto devemos salientar que um elemento particular da construção de $X_{K}^{F}$ desempenha um papel chave nesta identificação, a saber, a inclusão das marcas de $\mathcal{R}_{K}^{F}$. Isso garante que cada intervalo de constância $I$ de $\mathcal{I}_{k-1}^{F}$ tem ao menos uma marca (na verdade colocada em sua extremidade direita, que de fato não pertence a ele, mas devemos imaginar essa marca como ligada a ele). Sem essas marcas, o salto de $\left.X_{K}^{F}\right|_{K-1}$ pode não coincidir com aquele de $X_{K-1}^{F}$ — isso aconteceria se (e somente se) o primeiro intervalo de $\mathcal{I}_{K-1}^{F}$ vizinho à direita de $I$ não tivesse marcas.

Pela hipótese de indução, $\left.X_{K-1}^{F} \sim Z_{K-1}^{F} \sim Z_{K}^{F}\right|_{K-1}$; claramente $X_{K, K}^{F} \sim Z_{K, K}^{F}$. Terminamos o argumento em dois passos. O primeiro é mostrar que

$$
\mathbb{P}\left(\bar{A}_{K-1}\right)=\mathbb{P}\left(A_{K-1}\right)=\frac{1}{1+M_{K} \gamma_{K-1}^{F}\left(\left.x\right|_{K-1}\right)},
$$

e então usar a hipótese. Mas (3.10) segue da falta de memória de $N_{I}$, onde $I$ é o elemento de $\mathcal{I}_{K-1}^{F}$ que contém $\varphi_{K}(t)$. Com efeito, considere o evento $D_{i}=\left\{\varphi_{K}^{F}(t)\right.$ é o $i$-ésimo ponto de $\mathcal{S}_{K}^{F} \cup \mathcal{R}_{K}^{F}$ em $I$ contando da esquerda\} e $p_{i}=\mathbb{P}\left(D_{i}\right)$. Então

$$
\mathbb{P}\left(\bar{A}_{K-1} \mid D_{i}\right)=\mathbb{P}\left(N_{I}=i \mid N_{I} \geq i\right)=\mathbb{P}\left(N_{I}=1\right)=\frac{1}{1+M_{K} \gamma_{K-1}^{F}\left(\left.x\right|_{K-1}\right)},
$$

que implica

$$
\mathbb{P}\left(\bar{A}_{K-1}\right)=\sum_{i \geq 1} \mathbb{P}\left(\bar{A}_{K-1} \mid D_{i}\right) p_{i}=\frac{1}{1+M_{K} \gamma_{K-1}^{F}\left(\left.x\right|_{K-1}\right)},
$$

e (3.10) segue. E o derradeiro passo é argumentar que, dado $\bar{A}_{K-1}^{c}$, então $\left.X_{K}^{F}\right|_{K-1}$ não se move, e temos somente o salto uniforme em $\mathcal{M}_{K}$ de $X_{K, K}^{F}$, o que concorda com o movimento correspondente de $Z_{K}^{F}$ dado $A_{K-1}^{c}$, o que é imediato. 


\section{O processo $\mathrm{K}$ numa árvore}

Processos $\mathrm{K}$ em $\mathbb{N}_{*}$ foram introduzidos em [12] no estudo de limites de modelos de armadilha no gráfico completo. Eles aparecem como limites de escala do modelo de armadilha de Bouchaud no gráfico completo. Abaixo apresentamos uma extensão desse modelo em $\mathbb{N}_{*}^{k}$, para o qual olharemos como as folhas de uma árvore com $k$ gerações, da mesma forma como é feito nos capítulos anteriores (veja o parágrafo de (2.3) acima). O objetivo é, da mesma forma como em [12], estabelecer resultados limite para os processos dos capítulos anteriores quando o volume diverge.

Para $j=1, \ldots, k$, seja $\gamma_{j}: \mathbb{N}_{*}^{j} \rightarrow(0, \infty), j=1, \ldots, k$, tal que, se fizermos

$$
\bar{\gamma}_{j}\left(\left.x\right|_{j}\right):=\gamma_{1}\left(\left.x\right|_{1}\right) \times \gamma_{2}\left(\left.x\right|_{2}\right) \times \ldots \times \gamma_{j}\left(\left.x\right|_{j}\right),
$$

então

$$
\sum_{\left.x\right|_{j} \in \mathbb{N}_{*}^{j}} \bar{\gamma}_{j}\left(\left.x\right|_{j}\right)<\infty
$$

Estendemos $\gamma_{j}$ a $\overline{\mathbb{N}}_{*}^{j}$ declarando

$$
\gamma_{j}\left(\left.x\right|_{j}\right)=0 \text { for }\left.x\right|_{j} \in \overline{\mathbb{N}}_{*}^{j} \backslash \mathbb{N}_{*}^{j} .
$$

Aqui abusamos da notação ao identificar $\bar{\gamma}_{j}(\{\cdot\}) \operatorname{com} \bar{\gamma}_{j}(\cdot)$.

Construíremos um processo $X_{k}$ em $\overline{\mathbb{N}}_{*}^{k}$ indutivamente, da mesma forma como no capítulo 3. Inicialmente definimos o processo $X_{1}$. Ele é cadeia de Markov a tempo contínuo sobre $\overline{\mathbb{N}}_{*}$ descrita como se segue.

Todas as variáveis aleatórias subsequentes neste capítulo estão definidas no espaço de probabilidade $\left(\Omega^{\prime}, \mathcal{A}, \mathbb{P}\right)$. Assim sejam $\mathcal{N}_{1}=\left\{\left(N_{r}^{\left(x_{1}, 1\right)}\right)_{r \geq 0}, x_{1} \in \mathbb{N}_{*}\right\}$ processos de Poisson de i.i.d. de taxa 1. Seja $\sigma_{i}^{x_{1}, 1}$ a $i$-ésima ocorrência de $N^{\left(x_{1}, 1\right)}, i \geq 1$. Chamaremos de $\mathcal{S}_{1}=\left\{\sigma_{i}^{\left(x_{1}, 1\right)} ; x_{1} \in \mathbb{N}_{*}, i \geq 1\right\}$ o conjunto de marcas do primeiro nível de $X_{k}$. Sejam $\mathcal{T}_{1}=\left\{T_{s}^{(1)}, s \in \mathbb{R}_{+}\right\}$variáveis aleatórias i.i.d. exponenciais de média 1. Assume-se a independência entre $\mathcal{N}_{1}$ e $\mathcal{T}_{1}$.

Para $s \in \mathcal{S}_{1}$, seja

$$
\xi_{1}(s)=x_{1} \text { se } s=\sigma_{i}^{x_{1}, 1} \text { para algum } x_{1} \in \mathbb{N}_{*} \text { e } i \geq 1 .
$$

Note que $\xi_{1}$ está bem definido quase certamente. Definamos agora uma medida $\mu_{1}$ em $\mathbb{R}_{+}$ como se segue

$$
\mu_{1}(\{s\})=\gamma_{1}\left(\xi_{1}(s)\right) T_{s}^{(1)}, \text { se } s \in \mathcal{S}_{1} \text {, e } \mu_{1}\left(\mathbb{R}_{+} \backslash \mathcal{S}_{1}\right)=0
$$


Para $r \geq 0$, seja

$$
\Gamma_{1}(r):=\mu_{1}([0, r])=\sum_{x_{1}=1}^{\infty} \sum_{s \in\left\{\sigma_{i}^{\left(x_{1}, 1\right)} ; i \geq 1\right\} \cap[0, r]} \gamma_{1}\left(x_{1}\right) T_{s}^{(1)}
$$

e, para $t \geq 0$, seja

$$
\varphi_{1}(t):=\Gamma_{1}^{-1}(t)=\inf \left\{r \geq 0: \Gamma_{1}(r)>t\right\}
$$

a inversa contínua à direita de $\Gamma_{1}$.

Observação 4.1. Observe que $\mu_{1}$ é uma medida puramente atômica cujo conjunto de átomos, $\mathcal{S}_{1}$, é enumerável e denso em $\mathbb{R}_{+}$. Essas propriedades implicam que $\Gamma_{1}: \mathbb{R}_{+} \rightarrow \mathbb{R}_{+}$é estritamente crescente e que sua imagem $\Gamma_{1}\left(\mathbb{R}_{+}\right)$é um conjunto cuja medida de Lebesgue é nula. Também pode ser verificada que $\varphi_{1}: \mathbb{R}_{+} \rightarrow \mathbb{R}_{+}$é contínua.

Definimos $X_{1}$ sobre $\overline{\mathbb{N}}_{*}$ como se segue. Para $t \geq 0$

$$
X_{1}(t)=\left\{\begin{array}{l}
\xi_{1}\left(\varphi_{1}(t)\right), \text { se } \varphi_{1}(t) \in \mathcal{S}_{1}, \\
\infty, \text { caso contrário. }
\end{array}\right.
$$

Chamemos de $\mathcal{R}_{1}$ a imagem de $\Gamma_{1}$, ou seja, $\mathcal{R}_{1}=\Gamma_{1}\left(\mathbb{R}_{+}\right)=\left\{t \geq 0 ; \Gamma_{1}(r)=t\right.$ para algum $r \geq 0\}$.

Proposição 4.1. Vale quase certamente que $X_{1}(t)=\infty$ se e só se $t \in \mathcal{R}_{1}$.

Demonstração. Dada uma realização de nosso processo, tome $t \in \mathcal{R}_{1}$. Como $\Gamma_{1}$ é estritamente crescente há um único $r \geq 0$ tal que $\Gamma_{1}(r)=t$. Suponha que $X_{1}(t)=x<\infty$, então existe $s=\sigma_{i}^{(x, 1)} \in \mathcal{S}_{1}$ tal que $\Gamma_{1}\left(\sigma_{i}^{(x, 1)}-\right) \leq t<\Gamma_{1}\left(\sigma_{i}^{(x, 1)}\right)$. Já que $\Gamma_{1}$ é estritamente crescente temos $r<\sigma_{i}^{(x, 1)}$. Mas isso significa $\Gamma_{1}(r)<\Gamma\left(\sigma_{i}^{(x, 1)}-\right)$, o que contradiz a suposição.

Por outro lado, tome $t \geq 0$ tal que $X_{1}(t)=\infty$ e defina $s_{t}=\inf \left\{r \geq 0 ; \Gamma_{1}(r)>t\right\}$. Por definição, $\Gamma_{1}\left(s_{t}-\right) \leq t$ e $\Gamma_{1}\left(s_{t}\right) \geq t$. Se $\Gamma_{1}\left(s_{t}\right)>t$, então temos $\Gamma_{1}\left(s_{t}-\right) \leq t<\Gamma_{1}\left(s_{t}\right)$ e como antes, pelo fato de que os pontos de descontinuidade de $\Gamma_{1}$ são dados pelas marcas de Poisson, $s_{t}=\sigma_{i}^{(x, 1)} \in \mathcal{S}_{1}$ para algum $x<\infty$, o que corresponde a dizer que $X_{1}(t)=x<\infty$, mas isso contradiz a suposição.

Suponhamos que $X_{j}$ está definida para $j=1, \ldots, \ell-1,2 \leq \ell \leq k$.

Definição 4.1. Um intervalo $I \subset \mathbb{R}_{+}$é um intervalo de constância de $X_{j}$ se tem comprimento positivo e

$$
X_{j}(r)=X_{j}(s) \text { para todo } r, s \in I \text {, e I é maximal. }
$$


A condição de maximalidade e a continuidade à direita de $X_{j}$ implicam que $I=[a, b)$ para algum $0 \leq a<b$. Agora estamos prontos para definir $X_{\ell}$.

Seja $\mathcal{I}_{\ell-1}$ a coleção de intervalos de constância de $X_{\ell-1}$. Sejam $\mathcal{N}_{\ell}=\left\{\left(N_{r}^{\left(x_{\ell}, \ell\right)}\right), r \geq 0\right.$, $\left.x_{\ell} \in \mathbb{N}_{*}\right\}$ processos de Poisson i.i.d. de taxa 1. Seja $\sigma_{i}^{x_{\ell}, \ell}$ a $i$-ésima ocorrência de $N^{\left(x_{\ell}, \ell\right)}, i \geq 1$. Chamaremos de $\mathcal{S}_{\ell}=\left\{\sigma_{i}^{\left(x_{\ell}, \ell\right)} ; x_{\ell} \in \mathbb{N}_{*}, i \geq 1\right\}$ o conjunto de marcas de Poisson do $\ell$-ésimo nível. Sejam $\mathcal{T}_{\ell}=\left\{T_{s}^{(\ell)}, s \in \mathbb{R}_{+}\right\}$variáveis aleatórias i.i.d. exponenciais de média 1. Assumese a independência entre $\mathcal{N}_{\ell}$ e $\mathcal{T}_{\ell}$ e e a independência de ambos em relação a $\mathcal{N}_{j}$ e $\mathcal{T}_{j}$ para $j<\ell$.

Para $s \in \mathcal{S}_{\ell}$, seja $\xi_{\ell}(s)=x_{\ell}$ se $s=\sigma_{j}^{\left(x_{\ell}, \ell\right)}$ para algum $x_{\ell} \in \mathbb{N}_{*}$ e $j \geq 1$. Observe que $\xi_{\ell}$ está bem definida quase certamente. Definamos agora uma medida $\mu_{\ell}$ on $\mathbb{R}_{+}$como se segue:

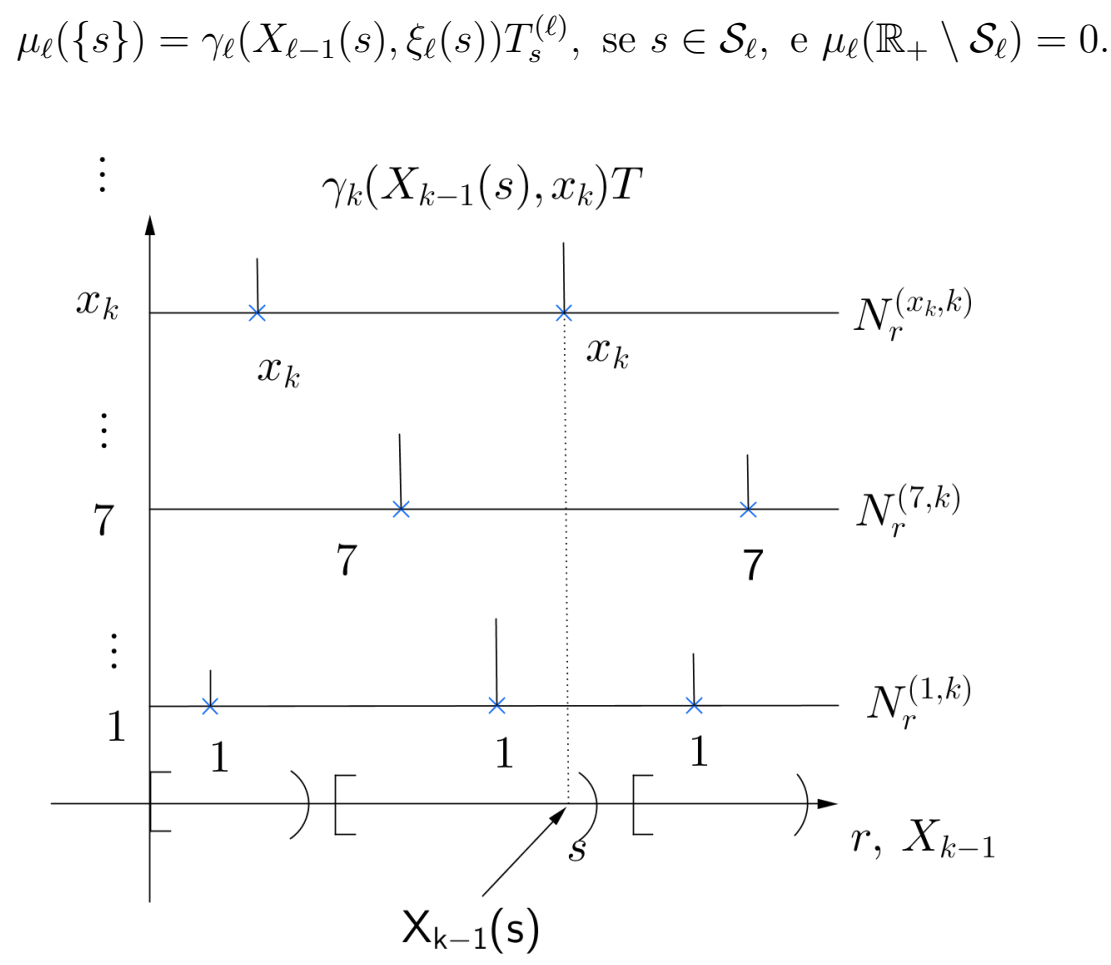

Figura 7: Marcas de Poisson para $k>1$.

Para $r \geq 0$, seja

$$
\Gamma_{\ell}(r):=\mu_{\ell}([0, r])
$$

e para $t \geq 0$, seja

$$
\varphi_{\ell}(t):=\Gamma_{\ell}^{-1}(t)=\inf \left\{r \geq 0 ; \Gamma_{\ell}(r)>t\right\}
$$


a inversa contínua à direita de $\Gamma_{\ell}$.

Observação 4.2. A Observação 4.1 vale com "1" substituído por " $\ell$ ". Em particular, a imagem de $\Gamma_{\ell}$ tem medida de Lebesgue nula, $\ell=1, \ldots, k$.

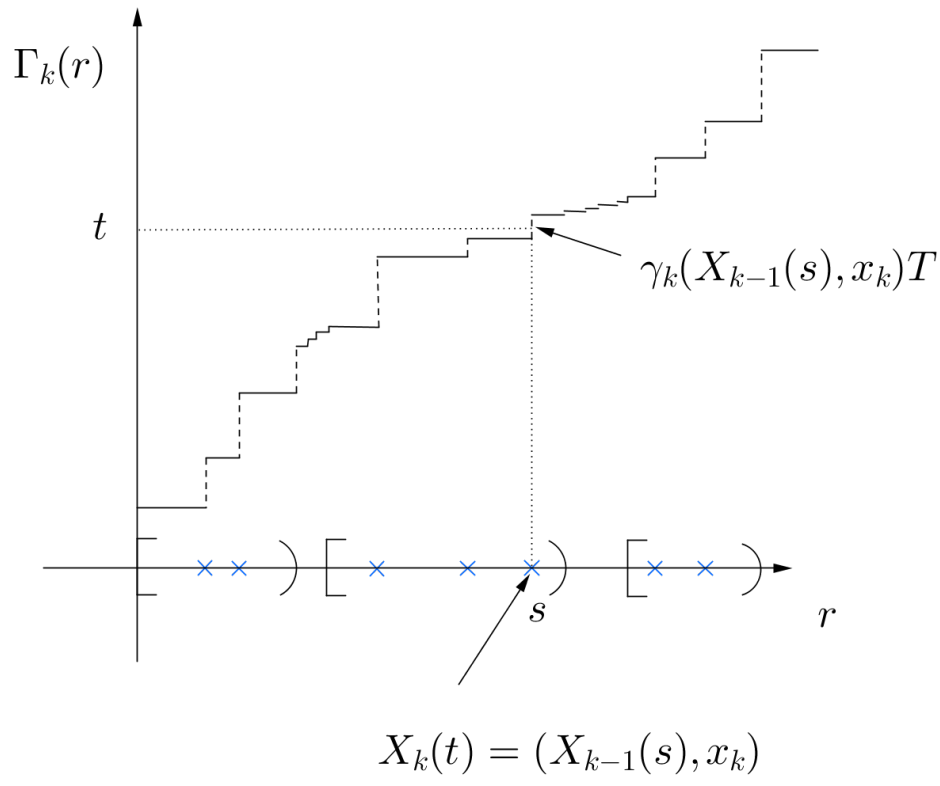

Figura 8: Uma realização de $X_{k}$.

Definimos o process $X_{\ell}$ sobre $\overline{\mathbb{N}}_{*}^{\ell}$ como se segue. Para $t \geq 0$, seja

$$
X_{\ell}(t)=\left\{\begin{array}{l}
\left(X_{\ell-1}\left(\varphi_{\ell}(t)\right), \xi_{\ell}\left(\varphi_{\ell}(t)\right)\right), \text { se } \varphi_{\ell}(t) \in \mathcal{S}_{\ell} \\
\left(X_{\ell-1}\left(\varphi_{\ell}(t)\right), \infty\right), \text { caso contrário. }
\end{array}\right.
$$

Definição 4.2. Chamamos de $X_{k}$ definido logo acima o processo $K$ em $\mathbb{T}_{k}$, ou processo com $k$ níveis, com conjunto de parâmetros $\underline{\gamma_{k}}=\left\{\gamma_{i} ; i=1, \ldots, k\right\}$.

Notação: $X_{k} \sim K_{k}\left(\mathbb{T}_{k}, \underline{\gamma_{k}}\right)$.

Observação 4.3. Segue das definições acima que $X_{1}(0)=\infty$ quase certamente e, consequentemente, que $X_{\ell}(0)=(\infty, \infty, \ldots, \infty)$ q.c..

O próximo resultado torna a construção acima bem definida. Para sua demonstração, introduzamos a notação

$$
X_{k}=\left(X_{k, 1}, \ldots, X_{k, k}\right), k \geq 1
$$

que torna explícitas as coordenadas de $X_{k}$. 
Lema 4.1. $\mathbb{P}$-quase certamente $\Gamma_{k}(r)<\infty$ para todo $r \in(0, \infty)$.

Demonstração. Como $\Gamma_{j}$ é não decrescente e ilimitada para $j=1, \ldots, k$, basta mostrar que $\Gamma_{k} \circ \Gamma_{k-1} \circ \ldots \circ \Gamma_{1}(r)<\infty$ para todo $r \in(0, \infty)$ quase certamente. Seja

$$
\Theta_{k}(r):=\Gamma_{k} \circ \Gamma_{k-1} \circ \ldots \circ \Gamma_{1}(r)
$$

Mostraremos por indução que

$$
\mathbb{E}\left(\Theta_{k}(r)\right)=r \sum_{x_{1}=1}^{\infty} \ldots \sum_{x_{k}=1}^{\infty} \gamma_{1}\left(\left.x\right|_{1}\right) \ldots \gamma_{k}\left(\left.x\right|_{k}\right)=r \sum_{x \in \mathbb{N}_{*}^{k}} \bar{\gamma}_{k}(x)
$$

que é a massa total de medida $\bar{\gamma}_{k}$, que por hipótese é finita (veja parágrafo de (4.2)), e isso obviamente encerra o argumento.

Isso é claramente verdade para $k=1$. Suponhamos que valha para $k-1, k \geq 2$. Consiremos os intervalos de constância de $X_{k, 1}$. Seja $\mathcal{I}=\left\{\right.$ intervalos de contância de $X_{k, 1} \subset$ $\left.\left[0, \Theta_{k}(r)\right]\right\}$. Podemos então enumerar os intervalos de $\mathcal{I}$ como $\mathcal{I}=\left\{I(s) ; s \in \mathcal{S}_{1} \cap[0, r]\right\}$. Assim,

$$
\Theta_{k}(r)=\sum_{s \in \mathcal{S}_{1} \cap[0, r]}|I(s)|=\sum_{x_{1}=1}^{\infty} \sum_{i_{1}=1}^{N_{r}^{\left(x_{1}\right)}} L_{i_{1}}^{\left(x_{1}\right)}
$$

onde $L_{i_{1}}^{\left(x_{1}\right)}:=\left|I\left(\sigma_{i_{1}}^{\left(x_{1}, 1\right)}\right)\right|$. Agora note que, para cada $x_{1} \in \mathbb{N}_{*}, N^{\left(x_{1}\right)}$ e $L^{\left(x_{1}\right)}:=\left\{L_{i_{1}}^{\left(x_{1}\right)}\right.$ : $\left.i_{1} \geq 1\right\}$ são independentes, e $L^{\left(x_{1}\right)}$ é uma família i.i.d. de variáveis aleatórias com $L_{i_{1}}^{\left(x_{1}\right)} \sim$ $\Theta_{k-1}^{\left(x_{1}\right)}\left(\gamma_{1}\left(x_{1}\right) T_{i_{1}}^{\left(x_{1}\right)}\right)$, onde $\Theta_{k-1}^{\left(x_{1}\right)}=\Gamma_{k-1} \circ \ldots \circ \Gamma_{1}$ é o correspondente de $\Theta_{k}$ para um processo $G K_{k-1}\left(\underline{\gamma_{k-1}^{\left(x_{1}\right)}}\right)$, onde $\underline{\gamma_{k-1}^{\left(x_{1}\right)}}=\left\{\gamma_{i}\left(x_{1}, \cdot\right): i=2, \ldots, k\right\}$.

\section{Então}

$$
\mathbb{E}\left(\Theta_{k}(r)\right)=r \sum_{x_{1}=1}^{\infty} \mathbb{E}\left\{\Theta_{k-1}^{\left(x_{1}\right)}\left(\gamma_{1}\left(\left.x\right|_{1}\right) T_{1}^{\left(x_{1}\right)}\right)\right\}=r \sum_{x_{1}=1}^{\infty} \gamma_{1}\left(\left.x\right|_{1}\right) \sum_{x_{2}, \ldots, x_{k}} \gamma_{2}\left(\left.x\right|_{2}\right) \ldots \gamma_{k}\left(\left.x\right|_{k}\right),
$$

onde usamos que $T_{i_{1}}^{\left(x_{1}\right)}, i_{1} \geq 1$ são exponenciais i.i.d. com média 1 e independentes de qualquer outra variável aleatória, e na segunda igualdade, a hipótese de indução.

Observação 4.4. Como podemos ver diretamente da Proposição 4.1, $X_{j, j}(t)=\infty$ para um dado $t$ se e só se $t \in \Gamma_{j}\left(\mathbb{R}_{+}\right)=\mathcal{R}_{j}$. Ademais, uma vez que $X_{j, i}(t)=\infty$ para algum $t \geq 0$ e $i=1, \ldots, j$, então $X_{j, l}(t)=\infty$ q.c. já que $\Gamma_{l} \circ \ldots \circ \Gamma_{i+1}\left(\mathcal{R}_{i}\right)$ é um conjunto de medida de Lebesgue nula e $\Gamma_{l} \circ \ldots \circ \Gamma_{i+1}\left(\mathcal{R}_{i}\right) \cap \mathcal{S}_{l}=\emptyset$ q.c. para $i+1 \leq l \leq j$. 


\section{Observação 4.5.}

1. Das Observações 4.2 e 4.4, segue que para $j=1, \ldots, k$, os infinitos de $X_{j}$, isto é, o conjunto $\left\{t \geq 0: X_{j, i}(t)=\infty\right.$ para algum $\left.i=1, \ldots, j\right\}$ tem medida de Lebesgue nula.

2. Segue que $X_{j, i}(s)<\infty$ para $s \in \mathcal{S}_{j+1}, i=1, \ldots, j$, e $j=1, \ldots, k-1$ quase certamente.

O próximo resultado afirma, grosso modo, que uma vez que uma coordenada de um processo K é grande, então também são as subsequentes coordenadas. Isso está de acordo com a propriedade mencionada na Observaï $i \frac{1}{2} \ddot{i} i \frac{1}{2}$ o 4.5.3. No próximo capítulo estabelecemos um resultado análogo em volume finito (veja Lema 5.3).

Lema 4.2. Seja $X_{k}$ o processo $K$ com $k$ níveis. Dados $\epsilon>0, T>0$ e $m \geq 1$, existem $m_{i}^{(k)}, i=1, \ldots, k-1$, tais que se $X_{k, i}(t)>m_{i}^{(k)}$ e $t \in[0, T]$, então $X_{k, j}(t)>m$ para todo $j=i+1, \ldots, k$ com $\mathbb{P}$-probabilidade maior que $1-\epsilon$.

Demonstração. Dado $\epsilon>0$, seja $T^{\prime}>0$ tal que $\mathbb{P}\left(\Theta_{k}\left(T^{\prime}\right)>T\right)>1-\epsilon / 3$, e para $m \in \mathbb{N}_{*}$ seja

$$
\tilde{\mathcal{S}}_{k}^{(m)}=\left\{\sigma_{i_{k}}^{\left(x_{k}, k\right)}: x_{k}=1, \ldots, m, i \geq 1\right\}
$$

Para $j=1, \ldots, k-1$ e $\ell \in \mathbb{N}_{*}$, seja $\mathcal{T}_{k-1, j}(\ell)=\left\{r \in\left(0, \Theta_{k-1}\left(T^{\prime}\right)\right) ; X_{k-1, j}(r)>\ell\right\}$. Já que $\left|\mathcal{T}_{k-1, j}(\ell)\right| \rightarrow \Theta_{k-1}\left(T^{\prime}\right)$ q.c. quando $\ell \rightarrow \infty$, pode-se ver prontamente das propriedades elementares de processos de Poisson que

$$
\left\{\cup_{j=1}^{k-1} \mathcal{T}_{k-1, j}(\ell)\right\} \cap \tilde{\mathcal{S}}_{k}^{(m)}=\emptyset
$$

quase certamente para todo $\ell$ suficientemente grande. Assim, dado $m \in \mathbb{N}$, podemos encontrar $m_{1}^{(k)}$ tal que se $X_{k, i}(t)>m_{1}^{(k)}, i=1, \ldots, k-1$, para qualquer $t \leq T$, então segue que $X_{k, k}(t)>m$ com probabilidade maior que $1-\epsilon / 3$. Isso em particular estabelece a afirmação para $k=2$ através da escolha $m_{1}^{(2)}$. Assumamos que a afirmação seja válida para $k-1 \mathrm{com}$ $\epsilon / 3$ em vez de $\epsilon$ e $T^{\prime \prime}$ em vez de $T$ tal que $\mathbb{P}\left(\Theta_{k-1}\left(T^{\prime}\right)<T^{\prime \prime}\right)>1-\epsilon / 3$. Então fazendo a escolha $m_{i}^{(k)}=m_{i}^{(k-1)} \vee m_{1}^{(k)}$, observamos que ela satisfaz a afirmação com probabilidade maior que $1-\epsilon$.

Lema 4.3. Seja $x=\left(x_{1}, \ldots, x_{k}\right) \in \overline{\mathbb{N}}_{*}^{k}$ tal que $x_{i}=\infty$ para algum $i \in\{1, \ldots, k\}$. Então $\mathbb{P}\left(X_{k}(t)=x\right)=0$ para Lebesgue quase todo $t>0$. 
Demonstração. Fixe $t>0$ e tome $T^{\prime}>0$ tal que, dado $\epsilon>0, \mathbb{P}\left(\Theta_{k}\left(T^{\prime}\right)>t\right)>1-\epsilon / 2 t$, onde $\Theta_{k}$ está definida em (4.10), e chame $F=\left\{\Theta_{k}\left(T^{\prime}\right)>t\right\}$. Para $m \geq 1$ e $j \in\{1, \ldots, k\}$, bote

$$
\tilde{\mathcal{S}}_{j}^{(m) c}=\left\{\sigma_{i}^{(x, j)}: x>m, i \geq 1\right\}
$$

e como antes defina

$$
\nu_{j}^{(m)}(\{s\})=\gamma_{j}\left(X_{j-1}(s), \xi_{j}(s)\right) T_{s}^{(j)}, \text { se } s \in \tilde{\mathcal{S}}_{j}^{(m) c}, \text { e } \nu_{j}^{(m)}\left(\mathbb{R}_{+} \backslash \tilde{\mathcal{S}}_{j}^{(m) c}\right)=0
$$

e para $r \geq 0$

$$
H_{j}^{(m)}(r):=\nu_{j}^{(m)}([0, r])
$$

Usaremos a mesma estratégia empregado para obter a igualdade em (4.11).

Se $x_{1}=\infty$, temos

$$
\int_{0}^{t} \mathbb{1}_{x}\left(X_{k}(z)\right) d z \leq \Gamma_{k} \circ \ldots \circ \Gamma_{2} \circ H_{1}^{(m)}\left(T^{\prime}\right) \mathbb{1}_{F}+t \mathbb{1}_{F^{c}}
$$

onde $\Gamma_{k} \circ \ldots \circ \Gamma_{2} \circ H_{1}^{(m)}\left(T^{\prime}\right)$ equivale ao tempo passado por $X_{k}$ de 0 até $\Theta_{k}\left(T^{\prime}\right)$ em estados $z$ cuja primeira coordenada $z_{1}$ é maior que $m$. Também notamos que $\Gamma_{k} \circ \ldots \circ \Gamma_{2} \circ H_{1}^{(m)}\left(T^{\prime}\right)$ tende a 0 quase certamente quando $m \rightarrow \infty$. Aplicando o teorema de Fubini obtemos

$$
\begin{aligned}
\mathbb{E}\left(\int_{0}^{t} \mathbb{1}_{x}\left(X_{k}(z)\right) d z\right) & =\int_{0}^{t} \mathbb{P}\left(X_{k}(z)=x\right) d z \\
& \leq \mathbb{E}\left(\Gamma_{k} \circ \ldots \circ \Gamma_{2} \circ H_{1}^{(m)}(r)\right)+t \mathbb{P}\left(F^{c}\right) \\
& \leq T^{\prime} \sum_{x_{1}>m} \sum_{x_{2}=1}^{\infty} \ldots \sum_{x_{k}=1}^{\infty} \bar{\gamma}_{k}\left(\left.x\right|_{k}\right)+\epsilon / 2 .
\end{aligned}
$$

Por (4.2) a soma no primeiro termo do membro direito de (4.18) é menor que $\epsilon / 2 T^{\prime}$ para $m$ suficientemente grande, logo o membro esquerdo de (4.18) é menor que $\epsilon$, o que implica $\mathbb{P}\left(X_{k}(t)=x\right)=0$ para quase todo $t>0$.

Se $x_{1}<\infty$, seja $a=\min \left\{i ; x_{i}=\infty\right\}$. Já que $\left.x\right|_{a-1} \in \mathbb{N}_{*}^{a-1}$, então existe um conjunto q.c. finito (um conjunto de marcas de Poisson) $\mathcal{A} \subset \mathcal{S}_{a-1} \cap\left[0, \Theta_{a-2}\left(T^{\prime}\right)\right]$ tal que para cada $s \in \mathcal{A}$ temos $\left.\mu_{a-1}(\{s\})=\gamma_{a-1}\left(\left.x\right|_{a-1}\right)\right) T_{s}^{(a-1)}$ e $\mathbb{E}(|\mathcal{A}|)=T^{\prime} \gamma_{1}\left(x_{1}\right) \ldots \gamma_{a-1}\left(\left.x\right|_{a-1}\right)$. Então

$$
\int_{0}^{t} \mathbb{1}_{x}\left(X_{k}(z)\right) d z \leq \sum_{s \in \mathcal{A}} \Gamma_{k} \circ \ldots \circ \Gamma_{a+1} \circ H_{a}^{(m)}\left(\gamma_{j}\left(\left.x\right|_{a-1}\right) T_{s}^{(a-1)}\right) \mathbb{1}_{F}+t \mathbb{1}_{F^{c}}
$$


para cada $m \geq 1$. Observe novamente que $\Gamma_{k} \circ \ldots \circ \Gamma_{a+1} \circ H_{a}^{(m)}(r) \rightarrow 0$ quase certamente quando $m \rightarrow \infty$ para cada $r>0$ e que pelo teorema de Fubini

$$
\begin{aligned}
\mathbb{E}\left(\int_{0}^{t} \mathbb{1}_{x}\left(X_{k}(z)\right) d z\right) & =\int_{0}^{t} \mathbb{P}\left(X_{k}(z)=x\right) d z \\
& \leq T^{\prime} \bar{\gamma}_{a-1}\left(\left.x\right|_{a-1}\right) \sum_{x_{a}>m} \sum_{x_{a+1}=1}^{\infty} \ldots \sum_{x_{k}=1}^{\infty} \gamma_{a}\left(\left.x\right|_{a}\right) \ldots \gamma_{k}\left(\left.x\right|_{k}\right)+\epsilon / 2,
\end{aligned}
$$

e com o mesmo raciocínio aplicado a (4.18) concluímos que $\mathbb{P}\left(X_{k}(t)=x\right)=0$ para quase todo $t>0$. 


\section{Convergência:}

Neste capítulo consideramos uma sequência de modelos de armadilhas $X_{k}^{(n)}, n \geq 1$, numa sequência de árvores finitas $\mathbb{T}_{k}^{(n)}$, com volumes $M_{1}=M_{1}^{(n)}, \ldots, M_{k}=M_{k}^{(n)}$, e conjunto de parâmetros $\gamma_{k}^{(n)}$, respectivamente, and provamos a convergência em probabilidade ao processo K numa árvore $X_{k}$ para essa sequência sob a topologia de Skorohod em $D\left(\overline{\mathbb{N}}_{*}^{k},[0, \infty)\right.$ ), o espaço das funções càdlàg de $[0, \infty)$ a $\overline{\mathbb{N}}_{*}^{k}$. Recomendamos ao leitor o apêndice $\mathrm{A}$ a fim de encontrar a definição dessa métrica e um exemplo de como ela funciona. Como antecipado no fim do Capítulo 1, substituiremos o sobrescrito " $F$ " por " $(n)$ " em toda parte para enfatizar a dependência em $n$.

\section{$5.1 \quad$ Resultados}

Nesta seção anunciamos o principal resultado até aqui e, com o próposito de usar a métrica de Skorohod (veja (A.2)), começamos dotando $\overline{\mathbb{N}}_{*}^{k}$ da métrica

$$
d(x, y)=\max _{1 \leq j \leq k}\left|x_{j}^{-1}-y_{j}^{-1}\right|, x, y \in \overline{\mathbb{N}}_{*}^{k},
$$

onde $\infty^{-1}=0$, sob a qual $\left(\overline{\mathbb{N}}_{*}^{k}, d\right)$ é diretamente compacto e assim completo.

A fim de obter nosso resultado de convergência, imporemos as seguintes condições sobre os volumes e parâmetros. Para $j=1, \ldots, k$, suponha que quando $n \rightarrow \infty$

$$
\begin{gathered}
M_{j}^{(n)} \rightarrow \infty \\
\gamma_{j}^{(n)}(x) \rightarrow \gamma_{j}(x) \text { para cada } x \in \mathbb{N}_{*}^{j} \text { e } \sum_{x \in \overline{\mathbb{N}}_{*}^{j}} \bar{\gamma}_{j}^{(n)}(x) \rightarrow \sum_{x \in \overline{\mathbb{N}}_{*}^{j}} \bar{\gamma}_{j}(x),
\end{gathered}
$$

onde $\gamma_{j}, \bar{\gamma}_{j}$ são como no ínicio do Capítulo 4 e satisfazem $(4.2-4.3)$, e $\gamma_{j}^{(n)} \equiv 0$ em $\left.\mathbb{N}_{*}^{j} \backslash \mathcal{M}\right|_{j}$.

Nosso resultado necessitará de condições adicionais que parecerão bastante obscuras. Enunciamo-las agora e discutimo-las, juntamente com as outras acima, após estabelecermos o resultado de convergência. Nós ainda supomos que para $j=2, \ldots, k$

$$
\frac{1}{\prod_{q=2}^{j} M_{q}^{(n)}} \sum_{\left.x\right|_{j} \in \mathbb{N}_{*}^{j}} \gamma_{j}^{(n)}\left(\left.x\right|_{j}\right) \sum_{p=1}^{j-1} \prod_{q=2}^{p} M_{q}^{(n)} \gamma_{q-1}^{(n)}\left(\left.x\right|_{q-1}\right) \prod_{q=p+2}^{j}\left(1+M_{q}^{(n)} \gamma_{q-1}^{(n)}\left(\left.x\right|_{q-1}\right)\right) \rightarrow 0
$$

e

$$
\frac{1}{\prod_{q=2}^{j} M_{q}^{(n)}} \sum_{\left.x\right|_{j-1} \in \mathbb{N}_{*}^{j-1}} \sum_{p=1}^{j-1} \prod_{q=2}^{p} M_{q}^{(n)} \gamma_{q-1}^{(n)}\left(\left.x\right|_{q-1}\right) \prod_{q=p+2}^{j}\left(1+M_{q}^{(n)} \gamma_{q-1}^{(n)}\left(\left.x\right|_{q-1}\right)\right) \rightarrow 0
$$


quando $n \rightarrow \infty$, onde por convenção

$$
\prod_{q=2}^{1} M_{q}^{(n)}=\prod_{q=2}^{1}\left(1+M_{q}^{(n)} \gamma_{q-1}^{(n)}\left(\left.x\right|_{q-1}\right)\right)=\prod_{q=j+1}^{j} M_{q}^{(n)} \gamma_{q-1}^{(n)}\left(\left.x\right|_{q-1}\right)=1
$$

Estamos prontos para enunciar nosso resultado maior.

Teorema 5.1. Para $n \geq 1$, seja $X_{k}^{(n)}$ o modelo de armadilhas em $\mathbb{T}_{k}^{(n)}$, com volumes $M_{1}^{(n)}, \ldots, M_{k}^{(n)}$, e conjuntos de parâmetros $\gamma_{k}^{(n)}$, respectivamente, satisfazendo as condições (5.2-5.5). Seja $X_{k}$ o processo $K$ em $\mathbb{T}_{k}$ com conjunto de parâmetros $\underline{\gamma_{k}}$. Então $X_{k}^{(n)} \rightarrow X_{k}$ em $\mathbb{P}$-probabilidade no espaço de Skorohod quando $n \rightarrow \infty$.

Neste ponto explicamos o significado das condições (5.2-5.5). (5.2) quer dizer que fazemos o limite dos volumes ir a infinito. (5.3) implica que as contribuições vindas das marcas de Poisson para a construção de $X_{k}^{(n)}$ convergem uniformemente às respectivas contribuições das marcas (de Poisson) de $X_{k}$. (5.4-5.5) implicam a negligibilidade da contribuição total das marcas de $X_{k}^{(n)}$ (como será visto em detalhes abaixo).

\subsection{Preliminares}

Para provar o Teorema 5.1, consideraremos versões acopladas de $X_{k}^{(n)}$ e $X_{k}$ e mostraremos a convergência em probabilidade. O acoplamento será dado pelo uso dos mesmos processos de Poisson $\left\{N^{\left(x_{i}, i\right)}, x_{i} \in \mathbb{N}_{*}, i=1, \ldots, k\right\}$ e mesmas variáveis exponenciais $\left\{T_{s}^{(i)}, s \in \mathbb{R}^{+}, i=\right.$ $1, \ldots, k\}$ na construção de $X_{k}^{(n)}$ e $X_{k}$.

Como parte da prova do Teorema 5.1, mostraremos que a contribuição das marcas extras e seus descendentes para $X_{j}^{(n)}$ é negligenciável quando $n \rightarrow \infty$. Esse é conteúdo do Lema 5.1. Precisamente, seja $\mathcal{E}_{2}^{(n)}=\mathcal{R}_{2}^{(n)}$ e para $3 \leq i \leq k$

$$
\mathcal{E}_{i}^{(n)}=\mathcal{R}_{i}^{(n)} \cup\left\{s \in \mathcal{S}_{i}^{(n)}: \varphi_{i-1}^{(n)}(s) \in \mathcal{E}_{i-1}^{(n)}\right\}
$$

$\mathcal{E}_{i}^{(n)}$ representa as marcas extras do $i$-ésimo nível e os descendentes das marcas extras dos níveis anteriores ao $i$-ésimo nível (ou seja, marcas de Poisson que pertencem aos intervalo de constância originários de marcas extras de níveis anteriores ou descendentes de marcas extras de níveis antes desse).

Lema 5.1. Para cada $r>0$ and $j=2, \ldots, k$

a) $\min \left\{\xi_{j}^{(n)}(s): s \in \mathcal{E}_{j}^{(n)} \cap[0, r]\right\} \rightarrow \infty$ em $\mathbb{P}$-probabilidade quando $n \rightarrow \infty$. 
b) $\mu_{j}^{(n)}\left(\mathcal{E}_{j}^{(n)} \cap[0, r]\right) \rightarrow 0$ em $\mathbb{P}$-probabilidade quando $n \rightarrow \infty$.

Demonstração. Antes de provar os itens $a$ ) e $b$ ) precisamos de introduzir algumas ideias. Dado $x=x_{1} \ldots x_{k} \in \mathbb{N}_{*}^{k}$, observe que por (5.3) para $n$ suficientemente grande

$$
\sum_{s \in\left\{\sigma_{i}^{\left(x_{1}, 1\right)} ; i \geq 1\right\} \cap[0, r]} \gamma_{1}^{(n)}\left(x_{1}\right) T_{s}^{(1)} \geq \frac{\gamma_{1}\left(x_{1}\right)}{2} \sum_{s \in\left\{\sigma_{i}^{\left(x_{1}, 1\right)} ; i \geq 1\right\} \cap[0, r]} T_{s}^{(1)} \rightarrow \infty \text { q.c. quando } r \rightarrow \infty \text {. }
$$

Isso em particular mostra que $\Gamma_{1}^{(n)}(r) \rightarrow \infty$ a.s. as $r \rightarrow \infty$, uniformemente em $n$. Ponha $F^{(n)}\left(\left.x\right|_{j}\right)=\left\{s \in \mathcal{S}_{j}^{(n)} ; s=\sigma_{i}^{\left(x_{j}, j\right)}, i \geq 1\right.$, e $\left.X_{j-1}^{(n)}(s)=\left.x\right|_{j-1}\right\}$.

Segue diretamente do fato de que $N_{r}^{\left(x_{1}, 1\right)} \rightarrow \infty$ q.c. qunado $r \rightarrow \infty$ que $\left|F^{(n)}\left(\left.x\right|_{1}\right)\right|=\infty$ q.c. para todo $n$. Suponhamos que $\left|F^{(n)}\left(\left.x\right|_{j-1}\right)\right|=\infty$ q.c. para todo $n$. Então novamente por (5.3) para $n$ suficientemente grande

$$
\sum_{s \in F^{(n)}\left(\left.x\right|_{j-1}\right)} \gamma_{j-1}^{(n)}\left(\left.x\right|_{j-1}\right) T_{s}^{(j-1)} \geq \frac{\gamma_{j-1}\left(\left.x\right|_{j-1}\right)}{2} \sum_{s \in F^{(n)}\left(\left.x\right|_{j-1}\right)} T_{s}^{(j-1)}=\infty \text { q.c. }
$$

o que implica que, quando olhamos para o eixo auxiliar $r$ do $j$-ésimo nível, a soma dos intervalos de constância com rótulo $\left.x\right|_{j-1}$ é infinita. Combinando isso às propriedades dos processos de Poisson implica que $\left|F^{(n)}\left(\left.x\right|_{j}\right)\right|=\infty$ q.c. . Assim

$$
\Gamma_{j}^{(n)}(r) \geq \gamma_{j}^{(n)}\left(\left.x\right|_{j}\right) \sum_{s \in F^{(n)}\left(\left.x\right|_{j}\right) \cap[0, r]} T_{s}^{(j)} \geq \frac{\gamma_{j}\left(\left.x\right|_{j}\right)}{2} \sum_{s \in F^{(n)}\left(\left.x\right|_{j}\right) \cap[0, r]} T_{s}^{(j)} \rightarrow \infty \text { q.c. quando } r \rightarrow \infty
$$

uniformemente em $n$.

Para $r>0$, seja

$$
\Theta_{j}^{(n)}(r):=\Gamma_{j}^{(n)} \circ \Gamma_{j-1}^{(n)} \circ \ldots \circ \Gamma_{1}^{(n)}(r)
$$

Já que $\Gamma_{j}^{(n)}(r) \rightarrow \infty$ q.c. quando $r \rightarrow \infty$ para $j=1, \ldots, k$ uniformemente em $n$, basta considerar $\mathcal{E}_{j}^{(n)} \cap\left[0, \Theta_{j-1}^{(n)}(r)\right]$ em vez de $\mathcal{E}_{j}^{(n)} \cap[0, r]$. Defina

$$
N_{j}^{(n)}(r):=\left|\mathcal{E}_{j}^{(n)} \cap\left[0, \Theta_{j-1}^{(n)}(r)\right]\right|
$$

Uma avaliação de $N_{j}^{(n)}(r)$ terá um papel crucial na prova. Começamos com ela. Para avaliar a cardinalidade desse conjunto assim como sua contribuição a $\mu_{j}^{(n)}\left(\left[0, \Theta_{j-1}^{(n)}(r)\right]\right)$, iniciamos pela descrição da estrutura de $\mathcal{S}_{1}^{(n)}, \mathcal{S}_{j}^{(n)} \cup \mathcal{R}_{j}^{(n)}$ para $j=2, \ldots k$; ao mesmo tempo, rerrotularemos esses pontos convenientemente. 
Cada $s_{1} \in \mathcal{S}_{1}^{(n)}$ pode ser identificado com seu rótulo $\xi_{1}\left(s_{1}\right)$ e seu índice $i_{1}\left(s_{1}\right)=i_{1} \in \mathbb{N}_{*}$ para o qual $s_{1}=\sigma_{i_{1}}^{\left(x_{1}, 1\right)}$. A cada marca $s_{1} \equiv\left(x_{1}, i_{1}\right)$ de $\mathcal{S}_{1}^{(n)}$ corresponde um intervalo $I_{n}^{\left(x_{1}, i_{1}\right)}$ de $\mathbb{R}_{+}$de comprimento $L_{n}^{\left(x_{1}, i_{1}\right)}=\gamma_{1}^{(n)}\left(x_{1}\right) T^{\left(x_{1}, i_{1}\right)}$. Tais intervalos formam uma partição de $\mathbb{R}_{+}$, e as variáveis aleatórias envolvidas são independentes, quando variamos $s_{1}$.

Agora a cada $s_{1} \in \mathcal{S}_{1}^{(n)}$ correspondem de $\mathcal{S}_{2}^{(n)}$ que pertencem ao respectivo intervalo $I_{n}^{\left(x_{1}, i_{1}\right)}$, cuja cardinalidade é uma variável aleatória geométrica $G^{\left(x_{1}, i_{1}\right)}$ de média $M_{2} \gamma_{1}^{(n)}\left(x_{1}\right)$, mais uma marca de $\mathcal{R}_{2}^{(n)}$ na extremidade direita de $I_{n}^{\left(x_{1}, i_{1}\right)}$. Tal marca será identificada com $\left(x_{1},\left.i\right|_{2}\right)$, onde $\left(x_{1}, i_{1}\right)$ é o identificador de $s_{1}$, e $i_{2} \in\left\{1, \ldots, G^{\left(x_{1}, i_{1}\right)}+1\right\}$, e associamos a ele uma variável aleatória $U^{\left(x_{1},\left.i\right|_{2}\right)}$ com distribuição uniforme em $M_{2}$, o que corresponde a $\xi_{2}\left(s_{2}\right)$, para $\left(x_{1},\left.i\right|_{2}\right) \equiv s_{2} \in \mathcal{S}_{2}^{(n)} \cup \mathcal{R}_{2}^{(n)}$. Identificaremos a única marca de $\mathcal{R}_{2}^{(n)}$ na extremidade direita de $I_{n}^{\left(x_{1}, i_{1}\right)} \operatorname{com}\left(x_{1}, i_{1}, G^{\left(x_{1}, i_{1}\right)}+1\right)$. Note que dado $\mathcal{S}_{1}^{(n)}$, as variáveis $\left\{G^{\left(x_{1}, i_{1}\right)}, U^{\left(x_{1},\left.i\right|_{2}\right)}\right\}$ são independentes.

Procedemos agora indutivamente. Para $3 \leq j \leq k$, assumimos que identificamos cada marca de $\mathcal{S}_{j-1}^{(n)} \cup \mathcal{R}_{j-1}^{(n)} \operatorname{como}\left(x_{1},\left.i\right|_{j-1}\right)$, com $x_{1} \in \mathcal{M}_{1}, i_{1} \geq 1, i_{l}=1, \ldots, G^{\left(x_{1}, i_{l-1}\right)}+1$, $l=2, \ldots, j-1$, onde $G^{\left(x_{1},\left.i\right|_{l-1}\right)}$ é geométrica de média $M_{l} \gamma_{l-1}^{(n)}\left(x_{1}, U^{\left(x_{1},\left.i\right|_{2}\right)}, \ldots, U^{\left(x_{1},\left.i\right|_{l-1}\right)}\right)$, $\operatorname{com} U^{\left(x_{1},\left.i\right|_{j}\right)} \sim \operatorname{Uniforme}\left(\mathcal{M}_{j}\right)$. As variáveis aleatórias de

$$
\mathcal{U}_{j-1}:=\left\{U^{\left(x_{1},\left.i\right|_{l}\right)}: l=2, \ldots, j-1, x_{1}, i_{1}, \ldots, i_{l} \geq 1\right\}
$$

são independentes, e também são as de

$$
\left\{G^{\left(x_{1}, i_{l-1}\right)}: l=3, \ldots, j-1, x_{1}, i_{1}, \ldots, i_{l} \geq 1\right\}
$$

desde condicionemos a $\mathcal{U}_{j-1}$. Aqui $i_{j-1}=1+G^{\left(x_{1},\left.i\right|_{j-2}\right)} \operatorname{significa}\left(x_{1},\left.i\right|_{j-1}\right) \in \mathcal{R}_{j-1}^{(n)}$; caso contrário, pertence a $\mathcal{S}_{j-1}^{(n)}$. Então a cada marca $\left(x_{1},\left.i\right|_{j-1}\right)$ corresponde um intervalo $I_{n}^{\left(x_{1},\left.i\right|_{j-1}\right)}$ de $\mathbb{R}_{+}$de comprimento

$$
L_{n}^{\left(x_{1},\left.i\right|_{j-1}\right)}:=\gamma_{j-1}^{(n)}\left(x_{1}, U^{\left(x_{1},\left.i\right|_{2}\right)}, \ldots, U^{\left(x_{1},\left.i\right|_{j-1}\right)}\right) T^{\left(x_{1},\left.i\right|_{j-1}\right)}
$$

$\operatorname{com}\left\{T^{\left(x_{1},\left.i\right|_{j-1}\right)}\right\}$ v.a. i.i.d. exponenciais de média 1 independentes de $\left\{G^{\left(x_{1},\left.i\right|_{l-1}\right)}, U^{\left(x_{1},\left.i\right|_{l}\right)}\right\}$, $l=2, \ldots, j-1 . \quad\left\{I_{n}^{\left(x_{1},\left.i\right|_{j-1}\right)}\right\}$ é uma partição de $\mathbb{R}_{+}$. As marcas de $\mathcal{S}_{j}^{(n)} \in I_{n}^{\left(x_{1},\left.i\right|_{j-1}\right)}$, se houver, serão rotuladas $\left(x_{1},\left.i\right|_{j}\right), i_{j}=1, \ldots, G^{\left(x_{1},\left.i\right|_{j-1}\right)}$, com $G^{\left(x_{1},\left.i\right|_{j-1}\right)}$ uma variável aleatória geométrica de média $M_{j} \gamma_{j-1}^{(n)}\left(x_{1}, U^{\left(x_{1},\left.i\right|_{2}\right)}, \ldots, U^{\left(x_{1},\left.i\right|_{j-1}\right)}\right)$. Condicionadas a $\mathcal{U}_{j-1}$, as variáveis aleatórias em $\left\{G^{\left(x_{1},\left.i\right|_{j-1}\right)}\right\}$ são independentes. A marca de $\mathcal{R}_{j}^{(n)}$ localizada na extremidade direita de $I_{n}^{\left(x_{1},\left.i\right|_{j-1}\right)}$ é rotulada $\left(x_{1},\left.i\right|_{j-1}, 1+G^{\left(x_{1},\left.i\right|_{j-1}\right)}\right)$. Finalmente, atribuimos a cada $\left(x_{1},\left.i\right|_{j}\right)$ uma variável aleatória $U^{\left(x_{1}, i_{j}\right)}$ uniformemente distribuida em $\mathcal{M}_{j}$, correspondendo a $\xi_{j}\left(s_{j}\right)$, 
para $\left(x_{1},\left.i\right|_{j}\right) \equiv s_{j} \in \mathcal{S}_{j}^{(n)} \cup \mathcal{R}_{j}^{(n)}$, com $\left\{U^{\left(x_{1},\left.i\right|_{j}\right)}\right\}$ independentes e independentes da variáveis aleatórias prévias.

Com esta representação, temos as marcas de $\left(\mathcal{S}_{j}^{(n)} \cup \mathcal{R}_{j}^{(n)}\right) \cap\left[0, \Theta_{j-1}^{(n)}(r)\right], j=2, \ldots, k$, rotuladas $\left(x_{1},\left.i\right|_{j}\right), x_{1}=1, \ldots, M_{1} ; i_{1}=1, \ldots, N_{r}^{\left(x_{1}\right)} ; i_{l}=1, \ldots, G^{\left(x_{1}, i_{l-1}\right)}+1,2 \leq l \leq$ $j$, com $G^{\left(x_{1},\left.\right|_{l-1}\right)}$ geometricamente distribuídas com média $M_{2} \gamma_{1}\left(x_{1}\right)$, quando $l=2$, e $M_{l} \gamma_{l-1}\left(x_{1}, U^{\left(x_{1},\left.i\right|_{2}\right)}, \ldots, U^{\left(x_{1},\left.i\right|_{l-1}\right)}\right)$, quando $l=3, \ldots, j$, respectivamente. $U^{\left(x_{1},\left.i\right|_{l}\right)}$ é uniformemente distribuída em $\mathcal{M}_{l}, l=2, \ldots, j$. As variáveis aleatórias na família

$$
\mathcal{U}_{j}:=\left\{U^{\left(x_{1}, i_{l}\right)} ; x_{1}, i_{1}, \ldots, i_{l} \geq 1, l=2, \ldots, j\right\}
$$

são independentes, e também são as em

$$
\left\{G^{\left(x_{1}, i_{l-1}\right)} ; x_{1}, i_{1}, \ldots, i_{l} \geq 1, l=3, \ldots, j\right\}
$$

desde condicionemos a $\mathcal{U}_{j}$.

As marcas de $\mathcal{E}_{j}^{(n)} \cap\left[0, \Theta_{j-1}^{(n)}(r)\right]$ são aquelas para as quais $i_{l}=G^{\left(x_{1},\left.i\right|_{l-1}\right)}+1:=\tilde{G}^{\left(x_{1}, i_{l-1}\right)}$ para algum $l=2, \ldots, j$. Para escrever uma expressão para $N_{j}^{(n)}(r)$ primeiramente vemos $\mathcal{E}_{j}^{(n)} \cap\left[0, \Theta_{j-1}^{(n)}(r)\right]$ como uma floresta, ou seja, uma união disjunta de árvores, cujas árvores distintas têm as marcas rotuladas $\left(x_{1},\left.i\right|_{l-1}, \tilde{G}^{\left(x_{1},\left.i\right|_{l-1}\right)}\right), l=2, \ldots, j$, como raízes. O número de raízes no l-ésimo nível é

$$
\sum_{x_{1}=1}^{M_{1}} \sum_{i_{1}=1}^{N_{r}^{\left(x_{1}, 1\right)}} \sum_{i_{2}=1}^{G^{\left(x_{1}, i_{1}\right)}} \cdots \sum_{i_{l-1}=1}^{G^{\left(x_{1},\left.i\right|_{l-2}\right)}} 1
$$

onde $\sum_{i_{1}=1}^{G^{\left(x_{1}, i_{0}\right)}}=1$ e o termo $\sum_{x_{1}=1}^{M_{1}} \sum_{i_{1}=1}^{N_{r}^{\left(x_{1}, 1\right)}}$ é mantido em (5.8) para cada $l \in\{2, \ldots, j\}$, e a árvore de raiz $\left(x_{1},\left.i\right|_{l-1} \tilde{G}^{\left(x_{1},\left.i\right|_{l-1}\right)}\right)$ consiste da, além da raiz, marcas cujos rótulos formam o conjunto

$$
\mathfrak{T}^{\left(x_{1},\left.i\right|_{l-1}\right)}:=\left\{\left(x_{1},\left.i\right|_{l-1}, \tilde{G}^{\left(x_{1},\left.i\right|_{l-1}\right)}, i_{l+1}, \ldots, i_{j}\right), i_{m}=1, \ldots, \tilde{G}^{\left(x_{1},\left.i\right|_{m-1}\right)}, m=l+1, \ldots, j\right\} .
$$

Portanto o número de elementos de $\mathcal{E}_{j}^{(n)} \cap\left[0, \Theta_{j-1}^{(n)}(r)\right]$ nas folhas de $\mathfrak{T}^{\left(x_{1},\left.i\right|_{l-1}\right)}$ é

$$
\sum_{i_{l+1}=1}^{\tilde{G}^{\left(x_{1},\left.i\right|_{l}\right)}} \cdots \sum_{i_{j}=1}^{\tilde{G}^{\left(x_{1},\left.i\right|_{j-1}\right)}} 1
$$

e sua contribuição a $\mu_{j}^{(n)}\left(\left[0, \Theta_{j-1}^{(n)}(r)\right]\right)$ é

$$
\sum_{i_{l+1}=1}^{\tilde{G}^{\left(x_{1},\left.i\right|_{l}\right)}} \ldots \sum_{i_{j}=1}^{\tilde{G}^{\left(x_{1},\left.i\right|_{j-1}\right)}} \gamma_{j}\left(x_{1}, U^{\left(x_{1},\left.i\right|_{2}\right)}, \ldots, U^{\left(x_{1},\left.i\right|_{j}\right)}\right) T^{\left(x_{1},\left.i\right|_{j}\right)}
$$


onde $\left\{T^{\left(x_{1}, i_{j}\right)}\right\}$ são i.i.d. variáveis aleatórias exponenciais de média 1, independentes de todas as variáveis aleatórias. Assim, o tamanho de $\mathcal{E}_{j}^{(n)} \cap\left[0, \Theta_{j-1}^{(n)}(r)\right]$ é

$$
N_{j}^{(n)}(r)=\sum_{l=2}^{j} \sum_{x_{1}=1}^{M_{1}} \sum_{i_{1}=1}^{N_{r}^{\left(x_{1}, 1\right)}} \sum_{i_{2}=1}^{G^{\left(x_{1}, i_{1}\right)}} \cdots \sum_{i_{l-1}=1}^{G^{\left(x_{1},\left.i\right|_{l-2}\right)}} \sum_{i_{l+1}=1}^{\tilde{G}^{\left(x_{1},\left.i\right|_{l}\right)}} \cdots \sum_{i_{j}=1}^{\tilde{G}^{\left(x_{1},\left.i\right|_{j-1}\right)}} 1
$$

e sua contribuição a $\mu_{j}^{(n)}\left(\left[0, \Theta_{j-1}^{(n)}(r)\right]\right)$ é

$$
\begin{gathered}
\mu_{j}^{(n)}\left(\mathcal{E}_{j}^{(n)} \cap\left[0, \Theta_{j-1}^{(n)}(r)\right]\right)= \\
\sum_{l=2}^{j} \sum_{x_{1}=1}^{M_{1}} \sum_{i_{1}=1}^{N_{r}^{\left(x_{1}, 1\right)}} \sum_{i_{2}=1}^{G^{\left(x_{1}, i_{1}\right)}} \ldots \sum_{i_{l-1}=1}^{G^{\left(x_{1},\left.i\right|_{l-2}\right)}} \sum_{i_{l+1}=1}^{\tilde{G}^{\left(x_{1},\left.i\right|_{l}\right)}} \ldots \sum_{i_{j}=1}^{\left(x_{1}, i_{j-1}\right)} \gamma_{j}\left(x_{1}, U^{\left(x_{1},\left.i\right|_{2}\right)}, \ldots, U^{\left(x_{1},\left.i\right|_{j}\right)}\right) T^{\left(x_{1},\left.i\right|_{j}\right)},
\end{gathered}
$$

onde por convenção $\sum_{i_{l+1}=1}^{\tilde{G}^{\left(x_{1},\left.\right|_{l}\right)}}=1$ se $l+1>j$.

Ao condicionar em $N_{r}^{\left(x_{1}, 1\right)}, G^{\left(x_{1}, i_{1}\right)}, \ldots, \tilde{G}^{\left(x_{1}, i_{j-2}\right)}, U^{\left(x_{1},\left.i\right|_{2}\right)}, \ldots, U^{\left(x_{1},\left.i\right|_{j-2}\right)}$, e integrar nas variáveis aleatórias remanescentes, obtemos de (5.11)

$$
\sum_{l=2}^{j} \sum_{x_{1}=1}^{M_{1}} \ldots \sum_{i_{l-1}=1}^{G^{\left(x_{1},\left.i\right|_{l-2}\right)}} \sum_{i_{l+1}=1}^{\tilde{G}^{\left(x_{1}, i_{l}\right)}} \ldots \sum_{i_{j-1}=1}^{\tilde{G}^{\left(x_{1},\left.i\right|_{j-2}\right)}} \frac{1}{M_{j-1}} \sum_{x_{j-1}=1}^{M_{j-1}}\left(1+M_{j} \gamma_{j-1}\left(x_{1}, U^{\left(x_{1}, i_{1}\right)}, \ldots, U^{\left(x_{1}, i_{j-2}\right)}, x_{j-1}\right)\right)
$$

e procedendo indutivamente, achamos

$$
\mathbb{E}\left(N_{j}^{(n)}(r)\right)=\frac{r}{\prod_{q=2}^{j-1} M_{q}} \sum_{l=2}^{j} \sum_{\left.\left.x\right|_{j-1} \in \mathcal{M}\right|_{j-1}} \prod_{q=2}^{l-1} M_{q} \gamma_{q-1}^{(n)}\left(\left.x\right|_{q-1}\right) \prod_{q=l+1}^{j}\left(1+M_{q} \gamma_{q-1}^{(n)}\left(\left.x\right|_{q-1}\right)\right) .
$$

Similarmente,

$$
\begin{gathered}
\mathbb{E}\left(\mu_{j}^{(n)}\left(\mathcal{E}_{j}^{(n)} \cap\left[0, \Theta_{j-1}^{(n)}(r)\right]\right)\right)= \\
\frac{r}{\prod_{q=2}^{j} M_{q}} \sum_{l=2}^{j} \sum_{\left.\left.x\right|_{j} \in \mathcal{M}\right|_{j}} \gamma_{j}^{(n)}\left(\left.x\right|_{j}\right) \prod_{q=2}^{l-1} M_{q} \gamma_{q-1}^{(n)}\left(\left.x\right|_{q-1}\right) \prod_{q=l+1}^{j}\left(1+M_{q} \gamma_{q-1}^{(n)}\left(\left.x\right|_{q-1}\right)\right) .
\end{gathered}
$$

Agora estamos prontos para demonstrar nossas afirmações.

a) Fixe $j \in\{2, \ldots, k\}, r>0$ e $L>0$. Então, pela desigualdade de Jensen

$$
\begin{aligned}
& \mathbb{P}\left(\min \left\{\xi_{j}^{(n)}(s): s \in \mathcal{E}_{j}^{(n)} \cap\left[0, \Theta_{j-1}^{(n)}(r)\right]\right\}>L\right)=\sum_{i=0}^{\infty}\left(1-\frac{L}{M_{j}}\right)^{i} \mathbb{P}\left(N_{j}^{(n)}(r)=i\right) \\
& =\mathbb{E}\left[\left(1-\frac{L}{M_{j}}\right)^{N_{j}^{(n)}(r)}\right] \geq\left(1-\frac{L}{M_{j}}\right)^{\mathbb{E}\left(N_{j}^{(n)}(r)\right)} \geq c \exp \left(-L \frac{\mathbb{E}\left(N_{j}^{(n)}(r)\right)}{M_{j}}\right),
\end{aligned}
$$


onde a última desigualdade vale para cada $c \in(0,1)$ fixado contanto que $n$ seja suficientemente grande e se usa (5.2). Utilizando (5.13), o quociente no expoente acima é exatamente a expressão (5.5), que vai a 0 quando $n \rightarrow \infty$. Então

$$
\liminf _{n \rightarrow \infty} \mathbb{P}\left(\min \left\{\xi_{j}^{(n)}(s): s \in \mathcal{E}_{j}^{(n)} \cap\left[0, \Theta_{j-1}^{(n)}(r)\right]\right\}>L\right) \geq c \text { para todo } c \in(0,1),
$$

e a parte $a$ ) do lema segue.

b) Dado $\varepsilon>0$, pela desigualdade de Markov

$$
\mathbb{P}\left(\mu_{j}^{(n)}\left(\mathcal{E}_{j}^{(n)} \cap\left[0, \Theta_{j-1}^{(n)}(r)\right]\right)>\varepsilon\right) \leq \varepsilon^{-1} \mathbb{E}\left(\mu_{j}^{(n)}\left(\mathcal{E}_{j}^{(n)} \cap\left[0, \Theta_{j-1}^{(n)}(r)\right]\right)\right)
$$

e o resultado segue de (5.14) e (5.4).

Para os próximos resultados desta seção, supomos que $X_{k-1}^{(n)} \rightarrow X_{k-1}$ q.c. no espaço de Skorohod quando $n \rightarrow \infty$.

Observação 5.1. Se $X_{k-1}^{(n)} \rightarrow X_{k-1}$ q.c. quando $n \rightarrow \infty$ no espaço de Skorohod, então, pela Proposição A.1, temos que

$$
\lim _{n \rightarrow \infty} X_{k-1}^{(n)}(s)=\lim _{n \rightarrow \infty} X_{k-1}^{(n)}(s-)=X_{k-1}(s)
$$

para todo $s \geq 0$ que é ponto de continuidade de $X_{k-1}$.

Lema 5.2. Seja $r \in[0, \infty)$ fixado. Suponha que $X_{k-1}^{(n)} \rightarrow X_{k-1}$ q.c. quando $n \rightarrow \infty$. Então $\Gamma_{k}^{(n)}(r) \rightarrow \Gamma_{k}(r)$ em $\mathbb{P}$-probabilidade quando $n \rightarrow \infty$.

Demonstração. A estratégia é simples. Separamos a contribuição da marcas extras e marcas de Poisson com rótulos altos. A convergência do resto, principal (como se verá) contribuição, à correspondente contribuição do volume infinito é fácil de mostrar, já que há somente um número finito fixado de contribuições envolvidas. Mostrar a negligibilidade da contribuição total das marcas extras e de rótulo alto ou é fácil ou segue diretamente das condições estipuladas. Agora, os detalhes.

Seja $\Psi_{k}^{(n)}(r)=\mu_{k}^{(n)}\left([0, r] \cap\left(\mathcal{S}_{k}^{(n)} \backslash \mathcal{E}_{k}^{(n)}\right)\right)$. Então

$$
\left|\Gamma_{k}(r)-\Gamma_{k}^{(n)}(r)\right| \leq\left|\Gamma_{k}(r)-\Psi_{k}^{(n)}(r)\right|+\mu_{k}^{(n)}\left([0, r] \cap \mathcal{E}_{k}^{(n)}\right)
$$

Sabemos pelo Lema 5.1b) que o segundo termo tende a 0 em probabilidade quando $n \rightarrow \infty$. Resta provar que o primeiro vai a 0 também. Relembre a notação $\left.x\right|^{i}=x_{i} \ldots x_{k}$ e de 
novo ponha $\tilde{\mathcal{S}}_{k}^{(m)}=\left\{\sigma_{i}^{(x, k)} ; x=1, \ldots, m, i \geq 1\right\}$. Assim, dado $\varepsilon>0$ e por (4.2), escolha $m_{1}, m_{2}, \ldots, m_{k} \in \mathbb{N}_{*}$ tal que

$$
\begin{gathered}
\sum_{x_{1}>m_{1}} \sum_{\left.x\right|^{2} \in \mathbb{N}_{*}^{k-1}} \bar{\gamma}_{k}\left(\left.x\right|_{k}\right)<\varepsilon / k, \\
\sum_{x_{1}=1}^{m_{1}} \sum_{x_{2}>m_{2}} \sum_{\left.x\right|^{3} \in \mathbb{N}_{*}^{k-2}} \bar{\gamma}_{k}\left(\left.x\right|_{k}\right)<\varepsilon / k, \\
\vdots \\
\sum_{x_{1}=1}^{m_{1}} \ldots \sum_{x_{k-1}=1}^{m_{k-1}} \sum_{x_{k}>m_{k}}^{\infty} \bar{\gamma}_{k}\left(\left.x\right|_{k}\right)<\varepsilon / k .
\end{gathered}
$$

Em seguida observe que

$$
\begin{array}{r}
\left|\Gamma_{k}(r)-\Psi_{k}^{(n)}(r)\right| \leq\left|\sum_{s \in \tilde{\mathcal{S}}_{k}^{\left(m_{k}\right)} \cap[0, r]}\left\{\gamma_{k}\left(X_{k-1}(s), \xi_{k}(s)\right)-\gamma_{k}^{(n)}\left(X_{k-1}^{(n)}(s), \xi_{k}(s)\right)\right\} T_{s}^{(k)}\right| \\
+\sum_{s \in \mathcal{S}_{k} \backslash \tilde{\mathcal{S}}_{k}^{\left(m_{k}\right)} \cap[0, r]} \gamma_{k}\left(X_{k-1}(s), \xi_{k}(s)\right) T_{s}^{(k)}+\sum_{s \in\left(\mathcal{S}_{k}^{(n)} \backslash\left(\tilde{\mathcal{S}}_{k}^{\left(m_{k}\right)} \cup \mathcal{R}_{k}^{(n)}\right)\right) \cap[0, r]} \gamma_{k}^{(n)}\left(X_{k-1}^{(n)}(s), \xi_{k}(s)\right) T_{s}^{(k)} .
\end{array}
$$

Mostraremos que os 3 componentes do membro direito de (5.19) convergem a 0 em probabilidade e então afirmação segue do teorema de Slutsky. De nossa hipótese, Lema A.1 e Proposição A.1, temos $X_{k-1}^{(n)}(s)=X_{k-1}(s)$ q.c. para todo $s \in \tilde{\mathcal{S}}_{k}^{m_{k}} \cap[0, r]$ para $n$ grande o suficiente. Então deduzimos que o primeiro termo à direita de (5.19) converge a 0 em probabilidade quando $n$ cresce pois é uma soma sobre uma quantia de termos quase certamente finita e $\gamma_{k}^{(n)}(\cdot) \rightarrow \gamma_{k}(\cdot)$. Seja

$$
B_{k}=\sum_{s \in \mathcal{S}_{k} \backslash \tilde{\mathcal{S}}_{k}^{\left(m_{k}\right)} \cap[0, r]} \gamma_{k}\left(X_{k-1}(s), \xi_{k}(s)\right) T_{s}^{(k)}
$$

e

$$
C_{k}^{(n)}=\sum_{s \in\left(\mathcal{S}_{k}^{(n)} \backslash\left(\tilde{\mathcal{S}}_{k}^{\left(m_{k}\right)} \cup \mathcal{R}_{k}^{(n)}\right)\right) \cap[0, r]} \gamma_{k}^{(n)}\left(X_{k-1}^{(n)}(s), \xi_{k}(s)\right) T_{s}^{(k)} .
$$

Agora tome $r_{B}>0$ tal que, dado $\epsilon>0, \mathbb{P}\left(\Theta_{k-1}\left(r_{B}\right)>r\right)>1-\epsilon$, e defina os subsequentes conjuntos

$$
\begin{gathered}
\mathcal{A}_{0}=\left(\mathcal{S}_{k} \backslash \tilde{\mathcal{S}}_{k}^{\left(m_{k}\right)}\right) \cap\left[0, \Theta_{k-1}\left(r_{B}\right)\right] \\
\mathcal{A}_{1}=\left\{s \in \mathcal{A}_{0}: X_{k-1,1}(s)>m_{1}\right\} \\
\mathcal{A}_{2}=\left\{s \in \mathcal{A}_{0}: X_{k-1,1}(s) \leq m_{1} \text { e } X_{k-1,2}>m_{2}\right\}, \ldots, \\
\mathcal{A}_{k}=\left\{s \in \mathcal{A}_{0}: X_{k-1,1}(s) \leq m_{1}, \ldots, X_{k-1, k-1} \leq m_{k-1}\right\}
\end{gathered}
$$


Em seguida ponha

$$
H_{k}=\sum_{i=1}^{k} \sum_{s \in \mathcal{A}_{i}} \gamma_{k}\left(X_{k-1}(s), \xi_{k}(s)\right) T_{s}^{(k)},
$$

e, dado $\delta>0$, chame $F_{B_{k}}=\left\{\Theta_{k-1}\left(r_{B}\right)>r\right\}$. Então

$$
\begin{aligned}
\mathbb{1}_{\left\{B_{k}>\delta\right\}} & =\mathbb{1}_{\left\{B_{k}>\delta\right\}} \mathbb{1}_{F_{B_{k}}}+\mathbb{1}_{\left\{B_{k}>\delta\right\}} \mathbb{1}_{F_{B_{k}}^{c}} \leq \mathbb{1}_{\left\{H_{k}>\delta\right\}} \mathbb{1}_{F_{B_{k}}}+\mathbb{1}_{F_{B_{k}^{c}}} \\
& \leq \mathbb{1}_{\left\{H_{k}>\delta\right\}}+\mathbb{1}_{F_{B_{k}}^{c}},
\end{aligned}
$$

o que implica $\mathbb{P}\left(B_{k}>\delta\right) \leq \mathbb{P}\left(H_{k}>\delta\right)+\epsilon$. Seguindo os mesmos argumentos usados para provar (4.11) e (5.18), temos

$$
\begin{gathered}
\mathbb{E}\left(\sum_{i=1}^{k} \sum_{s \in \mathcal{A}_{i}} \gamma_{k}\left(X_{k-1}(s), \xi_{k}(s)\right) T_{s}^{(k)}\right)= \\
r_{B}\left[\sum_{x_{1}>m_{1}} \sum_{\left.x\right|^{2} \in \mathbb{N}_{*}^{k-1}} \bar{\gamma}_{k}\left(\left.x\right|_{k}\right)+\sum_{\substack{x_{1}=1 \\
m_{1}}} \sum_{\substack{x_{2}>\left.m_{2} \\
x\right|^{3} \in \mathbb{N}_{*}^{k-2} \\
<r_{B} \varepsilon}} \bar{\gamma}_{k}\left(\left.x\right|_{k}\right)+\ldots+\sum_{x_{1}=1}^{m_{1}} \ldots \sum_{x_{k-1}=1}^{m_{k-1}} \sum_{x_{k}>m_{k}}^{\infty} \bar{\gamma}_{k}\left(\left.x\right|_{k}\right)\right]
\end{gathered}
$$

e da desigualdade de Markov concluimos que $B_{k} \rightarrow 0$ em probabilidade quando $m_{k} \rightarrow \infty$.

A fim demostrar que $C_{k}^{(n)} \rightarrow 0$ em probabilidade, mostremos que $\Gamma_{1}^{(n)}(s) \rightarrow \Gamma_{1}(s)$ em probabilidade para todo $s \in[0, \infty)$. Por causa do que fizemos até aqui, basta provar que $C_{1}^{(n)} \rightarrow 0$ em probabilidade quando $n \rightarrow \infty$. Assim

$$
C_{1}^{(n)}=\sum_{x_{1}>m} \sum_{s \in\left\{1, \ldots, N_{r}^{\left(x_{1}, 1\right)}\right\}} \gamma_{1}^{(n)}\left(x_{1}\right) T_{s}^{(1)},
$$

cuja média é $r \sum_{x_{1}>m} \gamma_{1}^{(n)}\left(x_{1}\right)$ vai a 0 uniformemente em $n$ por (5.3) quando $m \rightarrow \infty$, e isso completa a prova. Suponha agora que $\Gamma_{i}^{(n)}(s) \rightarrow \Gamma_{i}(s)$ em probabilidade para todo $s \in[0, \infty)$ para $1, \ldots, k-1$. Para $i=1, \ldots, k-1$, seja

$$
\Theta_{i}^{(n)}(s):=\Gamma_{i}^{(n)} \circ \Gamma_{i-1}^{(n)} \circ \ldots \circ \Gamma_{1}^{(n)}(s) .
$$

Dado $\varepsilon>0$, como $\Gamma_{k-1}^{(n)}(s) \rightarrow \Gamma_{k-1}(s)$ para todo $t \geq 0$, existe $r_{k-2}>0$ tal que $\Gamma_{k-1}^{(n)}\left(r_{k-2}\right)>r$ com probabilidade $\geq 1-\varepsilon / k$ para $n$ bastante grande. Igualmente para $i=1, \ldots, k-2$ há $r_{i-1}>0$ tal que $\Gamma_{i}^{(n)}\left(r_{i-1}\right)>r_{i}$ com probabilidade $\geq 1-\varepsilon / k$ para $n$ bastante grande. Portanto

$$
\mathbb{P}\left(\Theta_{k-1}^{(n)}\left(r_{0}\right)>r\right) \geq 1-\varepsilon \text { para } n \text { suficientemente grande }
$$


Com tal $r_{0}>0$ e escolha de $m_{1}, \ldots, m_{k}$ apresentada em (5.18), defina os conjuntos

$$
\begin{gathered}
\left.\mathcal{A}_{0}^{(n)}=\left(\mathcal{S}_{k}^{(n)} \backslash\left(\tilde{\mathcal{S}}_{k}^{\left(m_{k}\right)}\right) \cup \mathcal{R}_{k}^{(n)}\right)\right) \cap\left[0, \Theta_{k-1}^{(n)}\left(r_{0}\right)\right], \\
\mathcal{A}_{1}^{(n)}=\left\{s \in \mathcal{A}_{0}^{(n)}: X_{k-1,1}^{(n)}(s)>m_{1}\right\}, \\
\mathcal{A}_{2}^{(n)}=\left\{s \in \mathcal{A}_{0}^{(n)}: X_{k-1,1}^{(n)}(s) \leq m_{1} \text { and } X_{k-1,2}^{(n)}>m_{2}\right\}, \ldots, \\
\mathcal{A}_{k}^{(n)}=\left\{s \in \mathcal{A}_{0}^{(n)}: X_{k-1,1}^{(n)}(s) \leq m_{1}, \ldots, X_{k-1, k-1}^{(n)} \leq m_{k-1}\right\} .
\end{gathered}
$$

Aqui aplicaremos as mesmas ideias empregadas para mostrar que $B_{k} \rightarrow 0$ em probabilidade. Assim ponha

$$
\left.G_{k}^{(n)}=\sum_{i=1}^{k} \sum_{s \in \mathcal{A}_{i}^{(n)}} \gamma_{k}^{(n)}\left(X_{k-1}^{(n)}(s), \xi_{k}(s)\right) T_{s}^{(k)}\right)
$$

e, dado $\delta>0$, call $F_{C_{k}^{(n)}}=\left\{\Theta_{k-1}^{(n)}\left(r_{0}\right)>r\right\}$. Então

$$
\mathbb{1}_{\left\{C_{k}^{(n)}>\delta\right\}} \leq \mathbb{1}_{\left\{G_{k}^{(n)}>\delta\right\}}+\mathbb{1}_{\left(F_{C_{k}^{(n)}}\right)^{c}},
$$

o que implica $\mathbb{P}\left(C_{k}^{(n)}>\delta\right) \leq \mathbb{P}\left(G_{k}^{(n)}>\delta\right)+\epsilon$. Como consequência de (5.3), tome $n$ suficientemente grande tal que

$$
\begin{gathered}
\left|\sum_{x_{1}>m_{1}}^{M_{1}} \sum_{\left.\left.x\right|^{2} \in \mathcal{M}\right|^{2}} \bar{\gamma}_{k}^{(n)}\left(\left.x\right|_{k}\right)-\sum_{x_{1}>m_{1}} \sum_{\left.x\right|^{2} \in \mathbb{N}_{*}^{k-1}} \bar{\gamma}_{k}\left(\left.x\right|_{k}\right)\right|<\varepsilon / k, \\
\left|\sum_{x_{1}=1}^{m_{1}} \sum_{x_{2}=m_{2}+1}^{M_{2}} \sum_{\left.\left.x\right|^{3} \in \mathcal{M}\right|^{3}} \bar{\gamma}_{k}^{(n)}\left(\left.x\right|_{k}\right)-\sum_{x_{1}=1}^{m_{1}} \sum_{x_{2}>m_{2}} \sum_{\left.x\right|^{3} \in \mathbb{N}_{*}^{k-2}} \bar{\gamma}_{k}\left(\left.x\right|_{k}\right)\right|<\varepsilon / k, \\
\vdots \sum_{x_{1}=1}^{m_{1}} \ldots \sum_{x_{k-1}=1}^{m_{k-1}} \sum_{x_{k}=m_{k}+1}^{M_{k}} \bar{\gamma}_{k}^{(n)}\left(\left.x\right|_{k}\right)-\sum_{x_{1}=1}^{m_{1}} \ldots \sum_{x_{k-1}=1}^{m_{k-1}} \sum_{x_{k}>m_{k}} \bar{\gamma}_{k}\left(\left.x\right|_{k}\right) \mid<\varepsilon / k .
\end{gathered}
$$

Seguindo os mesmos argumentos usados para provar (4.11), (5.18) e (5.26), temos

$$
\begin{aligned}
& \mathbb{E}\left(\sum_{i=1}^{k-1} \sum_{s \in \mathcal{A}_{i}^{(n)}} \gamma_{k}^{(n)}\left(X_{k-1}^{(n)}(s), \xi_{k}(s)\right) T_{s}^{(k)}\right)= \\
& r_{0}\left[\sum_{x_{1}>m_{1}}^{M_{1}} \sum_{\left.\left.x\right|^{2} \in \mathcal{M}\right|^{2}} \bar{\gamma}_{k}^{(n)}\left(\left.x\right|_{k}\right)+\sum_{x_{1}=1}^{m_{1}} \sum_{x_{2}>m_{2}}^{M_{2}} \sum_{\left.\left.x\right|^{3} \in \mathcal{M}\right|^{3}} \bar{\gamma}_{k}^{(n)}\left(\left.x\right|_{k}\right)+\ldots+\sum_{x_{1}=1}^{m_{1}} \ldots \sum_{x_{k-1}=1}^{m_{k-1}} \sum_{x_{k}>m_{k}}^{M_{k}} \bar{\gamma}_{k}^{(n)}\left(\left.x\right|_{k}\right)\right] \\
& <r_{0} 2 \varepsilon,
\end{aligned}
$$


e da desigualdade de Markov concluimos que $C_{k}^{(n)} \rightarrow 0$ em probabilidade quando primeiramente fazemos $n \rightarrow \infty$ e após $m_{k} \rightarrow \infty$.

Corolário 5.1. O resultado do Lema 5.2 ainda vale se substituimos o $r$ do lado esquerdo por $r_{n}$ com $r_{n} \rightarrow r$ quando $n \rightarrow \infty$.

Demonstração. Escrevamos

$$
\left|\Gamma_{k}^{(n)}\left(r_{n}\right)-\Gamma_{k}(r)\right| \leq\left|\Gamma_{k}^{(n)}\left(r_{n}\right)-\Gamma_{k}^{(n)}(r)\right|+\left|\Gamma_{k}^{(n)}(r)-\Gamma_{k}(r)\right|
$$

O segundo termo de (5.27) vai a 0 em probabilidade quando $n \rightarrow \infty$ pelo Lema 5.2. Para provar que ocorre o mesmo com o primeiro, argumentamos como se segue. Dado $\eta>0$, já que $r$ é q.c. um ponto de continuidade de $\Gamma_{k}$, existe $\delta^{\prime}>0$ tal que $|r-s|<\delta^{\prime}$ implica $\left|\Gamma_{k}(r)-\Gamma_{k}(s)\right|<\eta / 2$ quase certamente.

Dada uma subsequência $(n ")$ de $(n)$ podemos achar outra subsequência $\left(n^{\prime}\right)$ para a qual o Lema 5.2 valha quase certamente. Como $\Gamma_{k}^{\left(n^{\prime}\right)}$ é não-decrescente e $\Gamma_{k}$ é crescente temos que para $n^{\prime}$ bastante grande $\left|r_{n^{\prime}}-r\right|<\delta^{\prime} / 4$ e $\Gamma_{k}^{\left(n^{\prime}\right)}\left(r_{n^{\prime}}\right), \Gamma_{k}^{\left(n^{\prime}\right)}(r) \in\left(\Gamma_{k}\left(r-\delta^{\prime} / 3\right), \Gamma_{k}\left(r+\delta^{\prime} / 3\right)\right)$ q.c.. Então

$$
\left|\Gamma_{k}^{\left(n^{\prime}\right)}\left(r_{n^{\prime}}\right)-\Gamma_{k}^{\left(n^{\prime}\right)}(r)\right| \leq \Gamma_{k}\left(r+\delta^{\prime} / 3\right)-\Gamma_{k}\left(r-\delta^{\prime} / 3\right)<\eta
$$

o que prova que $\Gamma_{k}^{\left(n^{\prime}\right)}\left(r_{n^{\prime}}\right) \rightarrow \Gamma_{k}(r)$ q.c. quando $n^{\prime} \rightarrow \infty$. Já que não há restrição sobre a primeira subsequência $(n ")$ a afirmação segue.

Observação 5.2. No resultado acima $r_{n}$ e r podem ser pensados como determinísticos, mas o mesmo argumento claramente funciona no caso aleatório contanto que $\left(r_{n}, r\right) e\left(\Gamma_{k}^{(n)}, \Gamma_{k}\right)$ sejam independentes $e r_{n} \rightarrow r$ q.c. quando $n \rightarrow \infty$. Isso pode ser aplicado para estabelecer que sob a assunção do Lema 5.2, temos que

$$
\Theta_{k}^{(n)}(T) \rightarrow \Theta_{k}(T) \text { as } n \rightarrow \infty
$$

em probabilidade para cada $T \geq 0$.

O seguinte resultado é uma versão em volume finito do Lema 4.2 no capítulo acima. 
Lema 5.3. Seja $X_{j}^{(n)}$ o modelo de armadilhas em $\mathbb{T}_{j}^{(n)}, j, n \geq 1$. Para $k \geq 2$ fixado, suponha que as hipóteses do Teorema 5.1 valham e que $X_{k-1}^{(n)} \rightarrow X_{k-1}$ q.c. quando $n \rightarrow \infty$. Então dados $m \geq 1$ e $\epsilon>0$, existem $m_{1}^{(k)}, \ldots, m_{k-1}^{(k)} \geq 1$ tais que o evento

$$
\begin{gathered}
A_{m}^{(n)}=\left\{\text { If } X_{k, i}^{(n)}(t)>m_{i}^{(k)} \text { para algum } i=1, \ldots, k-1 \text { e } t \in[0, T],\right. \\
\text { então } \left.X_{k, j}^{(n)}(t)>m \text { para } j=i+1, \ldots, k .\right\}
\end{gathered}
$$

tem probabilidade limitada inferiormente por $1-\epsilon$ uniformemente em $n$.

Demonstração. Argumentamos como na prova do Lema 4.2. Pela Observação 5.2 e quase certa finitude de $\Theta_{j}(r)$ para cada $j$ e $r$, podemos escolher $T^{\prime}>0$ tal que $\mathbb{P}\left(\Theta_{k}^{(n)}\left(T^{\prime}\right)>T\right)>$ $1-\epsilon / 4$, uniformemente em $n$. Agora para $m \in \mathbb{N}$, seja

$$
\tilde{\mathcal{S}}_{k}^{(m)}=\left\{\sigma_{i}^{(x, j)}: x=1, \ldots, m ; i \geq 1\right\}
$$

Para $j=1, \ldots, k-1$ and $\ell \in \mathbb{N}_{*}$, seja $\mathcal{T}_{k-1, j}^{(n)}(\ell)=\left\{r \in\left(0, \Theta_{k-1}^{(n)}\left(T^{\prime}\right)\right) ; X_{k-1, j}^{(n)}(r)>\ell\right\}$. Segue do Lema 5.1.b que a contribuição das marcas extras e seus descendentes desaparecem em probabilidade quando $n \rightarrow \infty$. Isso, o fato que $\left|\mathcal{T}_{k-1, j}^{(n)}(\ell)\right| \rightarrow \Theta_{k-1}^{(n)}\left(T^{\prime}\right)$ q.c. quando $\ell \rightarrow \infty$ e (5.29) junto com propriedades elementares de processos de Poisson implicam

$$
\left\{\cup_{j=1}^{k-1} \mathcal{T}_{k-1, j}^{(n)}(\ell)\right\} \cap \tilde{\mathcal{S}}_{k}^{(m)}=\emptyset
$$

com probabilidade arbitrariamente grande ( maior que $1-\epsilon / 4$ para nosso propósito) contanto que $\ell$ é bastante grande, uniformemente em $n$.

Assim, dado $m \in \mathbb{N}$, encontramos $m_{1}^{(k)}$ (de fato tomamos o mesmo valor do Lema 4.2) tal que fora um evento de probabilidade menor que $\epsilon / 2$, uniformemente em $n$, se $X_{k, i}^{(n)}(t)>m_{1}^{(k)}$, $i=1, \ldots, k-1$, para qualquer $t \leq T$, então $X_{k, k}^{(n)}(t)>m$. Isso em particular estabelece a afirmação para $k=2$ pela escolha $m_{1}^{(2)}$. Assumamos que a afirmação valha para $k-1$ (com $T^{\prime \prime}$ em vez de $T$ tal que $\mathbb{P}\left(\Theta_{k-1}^{(n)}\left(T^{\prime}\right)<T^{\prime \prime}\right)>1-\epsilon / 4$, uniformemente em $n$ ). Por nossa hipótese e Proposição A.1 vemos que $X_{k-1}^{(n)}(s)=X_{k-1}(s)$ q.c. para todo $s \in \tilde{\mathcal{S}}_{k}^{(m)} \cap\left[0, T^{\prime \prime}\right]$ para $n$ bastante grande. Como temos uma interseção de 4 conjuntos com probabilidade maior que $1-\epsilon / 4$, a escolha $m_{i}^{(k)}=m_{i}^{(k-1)} \vee m_{1}^{(k)}$ satisfaz o enunciado.

\subsection{Prova do Teorema 5.1}

Teorema 5.2 de [12] estabelece a convergência em probabilidade para $k=1$. A fim de provarmos o Teorema 5.1 para $k>1$, consideraremos versões acopladas de $X_{k}^{(n)}$ e $X_{k}$ e 
mostraremos convergência em probabilidade para uma subsequência. O acoplamento será dado pelo uso dos mesmos processos de Poisson $\left\{N^{\left(x_{i}, i\right)}, x_{i} \in \mathbb{N}_{*}, i=1, \ldots, k\right\}$ e variáveis exponenciais $\left\{T_{s}^{(i)}, s \in \mathbb{R}^{+}, i=1, \ldots, k\right\}$ na construção de $X_{k}^{(n)}$ e $X_{k}$. A notação está detalhada no começo do Capítulo 5, até o enunciado do Lema 5.1. Ficará claro que o mesmo pode ser feito para cada subsequência de $(n)$, e que o limite da subsequência não depende da subsequência. Isso então implica convergência em probabilidade da sequência original.

Para $j=1,2, \ldots, k$, sejam $X_{j}^{(n)} \sim T M_{j}\left(\mathbb{T}_{k}^{(n)}, \gamma_{j}^{(n)}\right)$ e $X_{j} \sim K_{j}\left(\mathbb{T}_{k}, \underline{\gamma_{j}}\right)$ e, para $k \geq 2$ fixado, suponha que $X_{k-1}^{(n)} \rightarrow X_{k-1}$ em probabilidade quando $n \rightarrow \infty$. Suporemos então indutivamente que

$$
X_{k-1}^{\left(n^{\prime}\right)} \rightarrow X_{k-1} \text { q.c. quando } n^{\prime} \rightarrow \infty
$$

para uma subsequência $\left(n^{\prime}\right)$. Dado $\epsilon>0$, lidemos com $T, T^{\prime}$ e $T^{\prime \prime}$ tais que $\mathbb{P}\left(\Theta_{k}\left(T^{\prime}\right)>T\right)>$ $1-\epsilon / 2$ e $\mathbb{P}\left(\Theta_{k-1}\left(T^{\prime}\right)<T^{\prime \prime}\right)>1-\epsilon / 2$. Recorde que $T^{\prime \prime}$ vem da hipótese de indução de Lema 4.2 e Lema 5.3. Escolheremos também $m_{i}^{(k)}, i=1, \ldots, k-1$, que satisfaçam as condições do Lema 4.2 e assuma que $m_{i}^{(k)} \geq m, i=1, \ldots, k-1$. Também assumiremos que as afirmações de Lema 5.1 e de (5.29) valham quase certamente sobre $\left(n^{\prime}\right)$.

Considere o conjunto $\mathcal{Y}=\left\{\Theta_{k}\left(T^{\prime}\right)>T\right\} \cap\left\{\Theta_{k}\left(T^{\prime}\right)<T^{\prime \prime}\right\}$. Dado $\delta>0$, mostraremos que para $\left(n^{\prime}\right)$

$$
\lim _{n^{\prime} \rightarrow \infty} \mathbb{P}\left(\left\{\rho\left(X_{k}, X_{k}^{\left(n^{\prime}\right)}\right)>\delta\right\} \cap \mathcal{Y}\right)=0
$$

onde $\rho$ é a métrica de Skorohod definida por (A.2), que implica $X_{k}^{\left(n^{\prime}\right)} \rightarrow X_{k}$ em probabilidade quando $n^{\prime} \rightarrow \infty$ visto que $\mathbb{P}\left(\mathcal{Y}^{c}\right)<\epsilon$.

Agora procedemos para definir uma distorção de tempo $\lambda^{\left(n^{\prime}\right)}$. Comecemos por considerar os intervalos de constância de $X_{k-1,1}$ em $\left[0, \Theta_{k-1}\left(T^{\prime}\right)\right)$ para os quais $X_{k-1,1} \leq m_{1}^{(k)}$. Esses são definidos os intervalos de constância da classe $m_{1}^{(k)}$ do primeiro nível para $X_{k-1}$. Continuando indutivamente, dado $2 \leq \ell \leq k-1$, para cada intervalo de constância $I$ de classe $m_{\ell-1}^{(k)}$ do nível $\ell-1$, consideramos os intervalos de constância de $X_{k-1, \ell}$ dentro de $I$ tais que $X_{k-1, \ell} \leq m_{\ell}^{(k)}$. A coleção de todos tais intervalos obtidos de todos intervalos de constância de classe $m_{\ell-1}^{(k)}$ do nível $\ell-1$ para $X_{k-1}$ forma o conjunto de intervalos de constância de classe $m_{\ell}^{(k)}$ do nível $\ell$ para $X_{k-1}$.

Denote $a_{1}, \ldots, a_{2 L}$ a coleção de todas as extremidades de todos os intervalos de constância de classe $m_{i}^{(k)}$ do nível $i$ para $X_{k-1}, i=1, \ldots, k-1$, em ordem crescente, e denote $b_{1}, \ldots, b_{2 J}$ a coleção de todas as extremidades de todos os intervalos de constância de classe $m_{k-1}^{(k)}$ do 
nível $k-1$ para $X_{k-1}$ em ordem crescente. Note que $\left\{b_{1}, \ldots, b_{2 J}\right\} \subset\left\{a_{1}, \ldots, a_{2 L}\right\}$. A razão para essa notação ficará abaixo.

Consideremos também os intervalos de constância de classe $m_{i}^{(k)}$ de nível $i$ para $X_{k-1}^{\left(n^{\prime}\right)}$, com definição paralela a de acima. Pela hipótese de que o Lema 5.1 vale quase certamente sobre $\left(n^{\prime}\right)$, e para $n^{\prime}$ grande o bastante, existe uma correspondência um-a-um entre $a_{1}^{\left(n^{\prime}\right)}, \ldots, a_{2 L^{\left(n^{\prime}\right)}}^{\left(n^{\prime}\right)}$ e $a_{1}, \ldots, a_{2 L}$, com $L^{\left(n^{\prime}\right)}=L$ para todo grande $n^{\prime}$ e $a_{i}^{\left(n^{\prime}\right)}$ corresponde a $a_{i}$, e de $(5.32)$,

$$
a_{i}^{\left(n^{\prime}\right)} \rightarrow a_{i}
$$

quando $n^{\prime} \rightarrow \infty$ para cada $i=1, \ldots, 2 L$.

Sejam $A_{i}=\Gamma_{k}\left(a_{i}\right)$ e $A_{i}^{\left(n^{\prime}\right)}=\Gamma_{k}^{\left(n^{\prime}\right)}\left(a_{i}^{\left(n^{\prime}\right)}\right), i=1, \ldots, 2 L$. Segue do Lema 5.2 que

$$
A_{i}^{\left(n^{\prime}\right)} \rightarrow A_{i}
$$

em probabilidade $n^{\prime} \rightarrow \infty$, e podemos assumir a convergência q.c. tomando uma subsequência.

Agora seja $\left\{s_{1}^{\prime}, \ldots, s_{Q}^{\prime}\right\}$ a enumeração em ordem crescente de $\cup_{i=1}^{J}\left\{\tilde{\mathcal{S}}_{k}^{(m)} \cap\left[b_{2 i-1}, b_{2 i}\right)\right\}$, e sejam $A_{i}^{-}=\Gamma_{k}\left(s_{i}^{\prime}-\right)$ e $A_{i}^{+}=\Gamma_{k}\left(s_{i}^{\prime}\right)$. Observamos que os intervalos $\left[A_{i}^{-}, A_{i}^{+}\right), i=1, \ldots, Q$ são intervalos de constância de classe $m_{k}^{(k)}$ de nível $k$ para $X_{k}$, com $m_{k}^{(k)}=m$, enquanto que $A_{i}, i=1, \ldots, 2 L$, são as extremidades de todos os intervalos de contância de classe $m_{i}^{(k)}$ de nível $i$ para $X_{k}, i=1, \ldots, k-1$.

Enfatizamos neste ponto que sob nossas hipótese até aqui, temos que para todo $i=$ $1, \ldots, J$

$$
\tilde{\mathcal{S}}_{k}^{(m)} \cap\left[b_{2 i-1}, b_{2 i}\right)=\tilde{\mathcal{S}}_{k}^{(m)} \cap\left[b_{2 i-1}^{\left(n^{\prime}\right)}, b_{2 i}^{\left(n^{\prime}\right)}\right)
$$

para todo $n^{\prime}$ suficientemente grande, onde $b_{i}^{\left(n^{\prime}\right)}=a_{j}^{\left(n^{\prime}\right)}$ tal que $a_{j}=b_{i}$.

Sejam $A_{i}^{-}\left(n^{\prime}\right)=\Gamma_{k}^{\left(n^{\prime}\right)}\left(s_{i}^{\prime}-\right), A_{i}^{+}\left(n^{\prime}\right)=\Gamma_{k}^{\left(n^{\prime}\right)}\left(s_{i}^{\prime}\right)$. Então para todo $n^{\prime}$ suficientemente grande, $\left[A_{i}^{-}\left(n^{\prime}\right), A_{i}^{+}\left(n^{\prime}\right)\right), i=1, \ldots, Q$ são os intervalos de constância de classe $m_{k}^{(k)}$ de nível $k$ para $X_{k}^{\left(n^{\prime}\right)}$, com $m_{k}^{(k)}=m$, enquanto que $A_{i}^{\left(n^{\prime}\right)}, i=1, \ldots, 2 L$, são as extremidades de todos os intervalos de constância de classe $m_{i}^{(k)}$ de nível $i$ para $X_{k}^{\left(n^{\prime}\right)}, i=1, \ldots, k-1$.

Argumentemos agora que

$$
A_{i}^{ \pm}\left(n^{\prime}\right) \rightarrow A_{i}^{ \pm}
$$

em probabilidade $n^{\prime} \rightarrow \infty$ (e de novo podemos assumir a convergência q.c. tomando uma subsequência). De fato basta primeiro notar que quase certamente $A_{i}^{-}\left(n^{\prime}\right)=\tilde{\Gamma}_{k}^{\left(n^{\prime}\right)}\left(s_{i}^{\prime}\right), A_{i}^{-}=$ $\tilde{\Gamma}_{k}\left(s_{i}^{\prime}\right), A_{i}^{+}\left(n^{\prime}\right)=\tilde{\Gamma}_{k}^{\left(n^{\prime}\right)}\left(s_{i}^{\prime}\right)+\gamma_{k}^{(n)}\left(X_{k-1}^{\left(n^{\prime}\right)}\left(s_{i}^{\prime}\right), \xi_{k}\left(s_{i}^{\prime}\right)\right) T_{s_{i}^{\prime}}^{(k)}$ e $A_{i}^{+}=\tilde{\Gamma}_{k}\left(s_{i}^{\prime}\right)+\gamma_{k}\left(X_{k-1}\left(s_{i}^{\prime}\right), \xi_{k}\left(s_{i}^{\prime}\right)\right) T_{s_{i}^{\prime}}^{(k)}$, 
onde $\tilde{\Gamma}_{k}^{\left(n^{\prime}\right)}$ e $\tilde{\Gamma}_{k}$ são obtidos de $\tilde{\mu}_{k}^{\left(n^{\prime}\right)}$ e $\tilde{\mu}_{k}$ como $\Gamma_{k}^{\left(n^{\prime}\right)}$ e $\Gamma_{k}^{\left(n^{\prime}\right)}$ são obtidos de $\mu_{k}^{\left(n^{\prime}\right)}$ e $\mu_{k}$, respectivamente, onde $\tilde{\mu}_{k}^{\left(n^{\prime}\right)}=\mu_{k}^{\left(n^{\prime}\right)}$ e $\tilde{\mu}_{k}=\mu_{k}$ em toda parte exceto em $s_{i}^{\prime}$, onde ambos $\tilde{\mu}_{k}^{\left(n^{\prime}\right)}$ e $\tilde{\mu}_{k}$ desaparecem. Pelos mesmos argumentos acima obtemos $\tilde{\Gamma}_{k}^{\left(n^{\prime}\right)}\left(s_{i}^{\prime}\right) \rightarrow \tilde{\Gamma}_{k}\left(s_{i}^{\prime}\right)$ em probabilidade, e o resultado segue, observando que $X_{k-1}^{\left(n^{\prime}\right)}\left(s_{i}^{\prime}\right)=X_{k-1}\left(s_{i}^{\prime}\right)$ para todo $n^{\prime}$ grande o suficiente e usando (5.3).

Estamos agora preparados para definir nossa distorção de tempo. Seja $\lambda^{\left(n^{\prime}\right)}:[0, \infty) \rightarrow$ $[0, \infty)$ tal que $\lambda^{\left(n^{\prime}\right)}(t)=\frac{A_{1}^{\left(n^{\prime}\right)}}{A_{1}} t$ se $t \in\left[0, A_{1}\right]$

$$
\lambda^{\left(n^{\prime}\right)}\left(A_{i}\right)=A_{i}^{\left(n^{\prime}\right)}, \quad \lambda^{\left(n^{\prime}\right)}\left(A_{i}^{-}\right)=A_{i}^{-}\left(n^{\prime}\right), \quad \lambda^{\left(n^{\prime}\right)}\left(A_{i}^{+}\right)=A_{i}^{+}\left(n^{\prime}\right),
$$

e faça-a linear entre pontos sucessivos de $\mathcal{A}:=\left\{A_{i}, i=1, \ldots, 2 L, A_{j}^{-}, A_{j}^{+}, j=1, \ldots, Q\right\}$, e linear com inclinação 1 de $\max \mathcal{A}$ em diante. Então $\lambda^{\left(n^{\prime}\right)}$ está bem definida para todo $n^{\prime}$ bastante grane e prontamente se vê que a condição (5.38) implica que $\lambda^{\left(n^{\prime}\right)}$ mapeia os intervalos de constância de classe $m_{i}^{(k)}$ de nível $i$ para $X_{k}$ aos correspondentes intervalos de constância de classe $m_{i}^{(k)}$ de nível $i$ para $X_{k}^{\left(n^{\prime}\right)}, i=1, \ldots, k$, dados pelo acoplamento. Em particular, $X_{k, j}\left(\lambda^{\left(n^{\prime}\right)}(\cdot)\right)=X_{k, j}^{\left(n^{\prime}\right)}(\cdot), j=1, \ldots i$, nesses respectivos intervalos. Das hipóteses do parágrafo de (5.32), temos então que (veja a expressão em (A.3))

$$
\sup _{0 \leq u \leq T} \rho\left(X_{k}^{\left(n^{\prime}\right)}, X_{k}, \lambda^{\left(n^{\prime}\right)}, u\right) \leq 1 / m
$$

e por (5.35) e (5.37) e nossa construção e hipóteses segue que (veja (A.1))

$$
\phi\left(\lambda^{\left(n^{\prime}\right)}\right) \rightarrow 0
$$

quando $n^{\prime} \rightarrow \infty$ para todo $\omega \in \mathcal{Y}$.

Segue de tudo acima que para cada $T>0$ fixado e $m \in \mathbb{N}$ encontramos uma subsequência $\left(n^{\prime}\right)$ tal que

$$
\limsup _{n^{\prime} \rightarrow \infty} \rho\left(X_{k}^{\left(n^{\prime}\right)}, X_{k}\right) \leq \frac{1}{m}+e^{-T}
$$

para todo $\omega \in \mathcal{Y}$, e isso diretamente implica que existe uma outra subsequência $\left(n^{\prime \prime}\right)$ tal que

$$
\lim _{n^{\prime \prime} \rightarrow \infty} \rho\left(X_{k}^{\left(n^{\prime \prime}\right)}, X_{k}\right)=0
$$

para todo $\omega \in \mathcal{Y}$. Isso prontamente implica (5.33), que por sua vez implica a afirmação do Teorema 5.1. 


\subsection{Medida invariante}

Provemos que sob as condições (5.2-5.5) o limite de

$$
\mathcal{G}_{k}^{(n)}(x)=\frac{\gamma_{k}^{(n)}(x)\left[\prod_{i=1}^{k-1} p_{i}^{(n)}\left(\left.x\right|_{i}\right)\right]^{-1}}{\sum_{\left.y \in \mathcal{M}\right|_{k} ^{(n)}} \gamma_{k}^{(n)}(y)\left[\prod_{i=1}^{k-1} p_{i}^{(n)}\left(\left.y\right|_{i}\right)\right]^{-1}},
$$

a expressão (2.8) na Observação 2.2 com " $(n)$ " em vez de " $F$ ", converge a medida invariante do process $\mathrm{K} X_{k}$ em $\mathbb{T}_{k}$ com conjunto de parâmetros $\underline{\gamma_{k}}$. Para $x \in \overline{\mathbb{N}}_{*}^{k}$, definamos

$$
\mathcal{G}_{k}(x)=\frac{\bar{\gamma}_{j}(x)}{\sum_{y \in \overline{\mathbb{N}}_{*}^{j}} \bar{\gamma}_{j}(y)} .
$$

Proposição 5.1. A medida dado por (5.44) é a medida invariante do processo $K X_{k}$ em $\mathbb{T}_{k}$ com conjunto de parâmetros $\underline{\gamma_{k}}$.

Antes de apresentarmos a prova da Proposição 5.1, anunciemos e provemos dois resultadois auxiliares.

Lema 5.4. Sob as hipóteses do Teorema 5.1, o processo $K$ com $k$ níveis é markoviano.

Demonstração. Dados $h, t>0$, fixamos $p \in \mathbb{N}_{*}$ e $0 \leq t_{1}<t_{2}<\ldots<t_{p}<t$. A prova do Teorema 5.1 nos dá a convergência em probabilidade, logo podemos tomar uma subsequência $\left(n^{\prime}\right)$ para a qual o Lema 5.1 e $X_{k}^{(n)} \rightarrow X_{k}$ na métrica de Skorohod valham q.c. quando $n^{\prime} \rightarrow \infty$. Para cada um dos pontos $t_{1}, \ldots, t_{p}, t, t+h$ suas coordenadas são ou todas finitas ou ao menos uma delas é $\infty$. No último caso o Lema 4.3 diz que q.c. isso ocorre com probabilidade zero. Assim a partir de agora consideramos todas as coordenadas finitas q.c., ou seja, para um ponto $t$ então $t \in I=\left[\Gamma_{k}(s-), \Gamma_{k}(s)\right)$, onde $s=\sigma_{i}^{\left(x_{k}, k\right)} \in \mathcal{S}_{k}$. Argumentemos agora que $t$ pertence ao interior de $I$. Dado que $\Gamma_{k}(s-)$ é uma variável aleatória contínua e uma realização de $\mathcal{S}_{k}$ tem um número enumerável de marcas, temos $\mathbb{P}\left(\Gamma_{k}(s-)=t \forall s \in \mathcal{S}_{k} \mid \mathcal{S}_{k}=u\right)=0$, o que por sua vez implica

$$
\mathbb{P}\left(\Gamma_{k}(s-)=t \forall s \in \mathcal{S}_{k}\right)=\int_{u} \mathbb{P}\left(\Gamma_{k}(s-)=t \forall s \in \mathcal{S}_{k} \mid \mathcal{S}_{k}=u\right) d \mathbb{P}\left(\mathcal{S}_{k}=u\right)=0,
$$

e afirmação está provada. Com isso em mente e pelo acoplamento de $X_{k}$ e $X_{k}^{(n)}$, é claro que se $X_{k}\left(t_{i}\right)=z_{i} \in \mathbb{N}_{*}^{k}$ então para $n^{\prime}$ suficientemente grande $X_{k}^{\left(n^{\prime}\right)}\left(t_{i}\right)=z_{i}, i=1, \ldots, p$. Logo temos $\left\{X_{k}(t+h)=x, X_{k}(t)=y, X_{k}\left(t_{i}\right)=z_{i}, i=1, \ldots, p\right\}=\left\{X_{k}^{\left(n^{\prime}\right)}(t+h)=x, X_{k}^{\left(n^{\prime}\right)}(t)=\right.$ 
$\left.y, X_{k}^{\left(n^{\prime}\right)}\left(t_{i}\right)=z_{i}, i=1, \ldots, p\right\}$ para $n^{\prime}$ bastante grande. Usando a propriedade de Markov de $X_{k}^{\left(n^{\prime}\right)}$, portanto, podemos escrever

$$
\begin{aligned}
& \mathbb{P}\left(X_{k}(t+h)=x \mid X_{k}(t)=y, X_{k}\left(t_{i}\right)=z_{i}, i=1, \ldots, p\right) \\
& =\mathbb{P}\left(X_{k}^{\left(n^{\prime}\right)}(t+h)=x \mid X_{k}^{\left(n^{\prime}\right)}(t)=y, X_{k}^{\left(n^{\prime}\right)}\left(t_{i}\right)=z_{i}, i=1, \ldots, p\right) \\
& =\mathbb{P}\left(X_{k}^{\left(n^{\prime}\right)}(t+h)=x \mid X_{k}^{\left(n^{\prime}\right)}(t)=y\right)=\mathbb{P}\left(X_{k}(t+h)=x \mid X_{k}(t)=y\right),
\end{aligned}
$$

o que estabelece o enunciado.

Lema 5.5. Seja $a_{i}>0, i=1, \ldots, k$. Então

$$
\prod_{i=1}^{k}\left(1+a_{i}\right)=\sum_{p=1}^{k+1} \prod_{i=1}^{p-1} a_{i} \prod_{i=p+1}^{k}\left(1+a_{i}\right) .
$$

Demonstração. Para $k=1$ a igualdade segue facilmente da convenção $\prod_{i=1}^{0} a_{i}=\prod_{i=p+1}^{k}(1+$ $\left.a_{i}\right)=1$ se $p+1>k$. Suponha que (5.46) valha para $k \geq 1$. Provemos que (5.46) vale para $k+1$.

$$
\begin{aligned}
& \sum_{p=1}^{k+2} \prod_{i=1}^{p-1} a_{i} \prod_{i=p+1}^{k+1}\left(1+a_{i}\right)=\sum_{p=1}^{k} \prod_{i=1}^{p-1} a_{i} \prod_{i=p+1}^{k}\left(1+a_{i}\right)\left(1+a_{k+1}\right)+\prod_{i=1}^{k} a_{i}+\prod_{i=1}^{k+1} a_{i} \\
& =\sum_{p=1}^{k} \prod_{i=1}^{p-1} a_{i} \prod_{i=p+1}^{k}\left(1+a_{i}\right)+\prod_{i=1}^{k} a_{i}+a_{k+1}\left\{\sum_{p=1}^{k} \prod_{i=1}^{p-1} a_{i} \prod_{i=p+1}^{k}\left(1+a_{i}\right)+\prod_{i=1}^{k} a_{i}\right\} \\
& =\sum_{p=1}^{k+1} \prod_{i=1}^{p-1} a_{i} \prod_{i=p+1}^{k}\left(1+a_{i}\right)+a_{k+1}\left\{\prod_{i=1}^{k}\left(1+a_{i}\right)\right\} \\
& =\left(1+a_{k+1}\right) \prod_{i=1}^{k}\left(1+a_{i}\right)=\prod_{i=1}^{k+1}\left(1+a_{i}\right) .
\end{aligned}
$$

Prova da Proposição 5.1. Considere $\mathbb{P}\left(X_{k}(t)=x \mid X_{k}(0)=y\right)=p_{y, x}(t)$. Como consequência do Lema 5.4, o Teorema 1 da Seção 1 da Parte II de [6] implica que $p_{y, x}(\cdot)$ é uma função contínua, logo podemos estender o Lema 4.3 para todo $t>0$. Para $x \in \overline{\mathbb{N}}_{*}^{k} \backslash \mathbb{N}_{*}^{k}$, Lema 4.3 e hipótese (4.3) implicam

$$
0=\mathcal{G}_{k}(x)=\sum_{y \in \overline{\mathbb{N}}_{*}^{k}} \mathcal{G}_{k}(y) p_{y, x}(t)
$$

para todo $t>0$. 
Agora considere $x \in \mathbb{N}_{*}^{k}$. Da Observação 2.2 sabemos que

$$
\mathcal{G}_{k}^{(n)}(x)=\sum_{\left.y \in \mathcal{M}\right|_{k} ^{(n)}} \mathcal{G}_{k}^{(n)}(y) p_{y, x}^{(n)}(t)
$$

onde $\mathbb{P}\left(X_{k}^{(n)}(t)=x \mid X_{k}^{(n)}(0)=y\right)=p_{y, x}^{(n)}(t)$.

Para obter a medida invariante de $X_{k}$, inicialmente mostraremos que $\mathcal{G}_{k}^{(n)}(x) \rightarrow \mathcal{G}_{k}(x)$ quando $n \rightarrow \infty$ e depois provaremos que a soma no lado direito de (5.47) tende a

$$
\sum_{y \in \overline{\mathbb{N}}_{*}^{k}} \mathcal{G}_{k}(y) p_{y, x}(t) \text { quando } n \rightarrow \infty
$$

Para $i=1, \ldots, k-1$, relembre que $p_{i}^{(n)}\left(\left.x\right|_{i}\right)=\frac{1}{1+M_{i+1}^{(n)} \gamma_{i}^{(n)}\left(\left.x\right|_{i}\right)}$ e assim (5.43) torna-se

$$
\mathcal{G}_{k}^{(n)}(x)=\frac{\gamma_{k}^{(n)}(x) \prod_{i=1}^{k-1}\left(1+M_{i+1}^{(n)} \gamma_{i}^{(n)}\left(\left.x\right|_{i}\right)\right)}{\sum_{\left.y \in \mathcal{M}\right|_{k} ^{(n)}} \gamma_{k}^{(n)}(y) \prod_{i=1}^{k-1}\left(1+M_{i+1}^{(n)} \gamma_{i}^{(n)}\left(\left.y\right|_{i}\right)\right)}
$$

Usando o Lema $5.5 \mathrm{com} i+1=q$, para qualquer $\left.y \in \mathcal{M}\right|_{k} ^{(n)}$ reescrevemos ambos os produtos no numerador e denominador de (5.49) como

$$
\sum_{p=1}^{k-1} \prod_{q=2}^{p} M_{q}^{(n)} \gamma_{q-1}^{(n)}\left(\left.y\right|_{q-1}\right) \prod_{q=p+2}^{k}\left(1+M_{q}^{(n)} \gamma_{q-1}^{(n)}\left(\left.y\right|_{q-1}\right)\right)+\prod_{q=2}^{k} M_{q}^{(n)} \gamma_{q-1}^{(n)}\left(\left.y\right|_{q-1}\right) .
$$

Note que o membro esquerdo de (5.50) é exatamente a expressão dentro da soma de (5.4) para $j=k$ (que vai a 0 ) e o limite do membro direito dividido por $\prod_{q=2}^{k} M_{q}^{(n)}$ é $\prod_{q=2}^{k} \gamma_{q-1}\left(\left.y\right|_{q-1}\right)$. Pelas condições (5.2-5.4), após dividirmos numerador e denominador de (5.49) por $\prod_{q=2}^{k} M_{q}^{(n)}$, temos

$$
\mathcal{G}_{k}^{(n)}(x) \rightarrow \mathcal{G}_{k}(x) \text { quando } n \rightarrow \infty
$$

Tratemos agora do membro direito de (5.47). Relembre a notação $\left.y\right|^{i}=y_{i} \ldots y_{k}$. Como na prova do Lema 5.2 , por (4.2), tomamos $m_{1}, \ldots, m_{k} \in \mathbb{N}_{*}$ tais que, dado $\varepsilon>0$,

$$
\sum_{i=1}^{k} \sum_{y_{1}=1}^{m_{1}} \ldots \sum_{y_{i-1}=1}^{m_{i-1}} \sum_{y_{i}>m_{i}} \sum_{\left.y\right|^{i+1} \in \mathbb{N}_{*}^{k-i}} \bar{\gamma}_{k}(y)<\varepsilon
$$

Em seguida dividimos o componente direito de (5.47) em $k+1$ termos para obter o seguinte limitante superior

$$
\sum_{y_{1}=1}^{m_{1}} \ldots \sum_{y_{k}=1}^{m_{k}} \mathcal{G}_{k}^{(n)}(y) p_{y, x}^{(n)}(t)+\sum_{i=1}^{k} \sum_{y_{1}=1}^{m_{1}} \ldots \sum_{y_{i-1}=1}^{m_{i-1}} \sum_{y_{i}>m_{i}} \sum_{\left.y\right|^{i+1} \in \mathbb{N}_{*}^{k-i}} \mathcal{G}_{k}^{(n)}(y) .
$$


Então olhamos para o termo direito de (5.52). Por (5.49), seu numerador pode ser escrito como

$$
\frac{1}{\prod_{i=1}^{k-1} M_{i+1}^{(n)}} \sum_{i=1}^{k} \sum_{y_{1}=1}^{m_{1}} \ldots \sum_{y_{i-1}=1}^{m_{i-1}} \sum_{y_{i}>m_{i}} \sum_{\left.y\right|^{i+1} \in \mathbb{N}_{*}^{k-i}} \gamma_{k}^{(n)}(y) \prod_{i=1}^{k-1}\left(1+M_{i+1}^{(n)} \gamma_{i}^{(n)}\left(\left.y\right|_{i}\right)\right)
$$

e assim seu denominador é

$$
\frac{1}{\prod_{i=1}^{k-1} M_{i+1}^{(n)}} \sum_{\left.y \in \mathcal{M}\right|_{k} ^{(n)}} \gamma_{k}^{(n)}(y) \prod_{i=1}^{k-1}\left(1+M_{i+1}^{(n)} \gamma_{i}^{(n)}\left(\left.y\right|_{i}\right)\right) .
$$

Aplicamos agora o mesmo raciocínio prévio. O Lema 5.5 e condições (5.2-5.4) implicam que (5.53) converge a $(5.51)$ e que (5.54) tende a $\sum_{y \in \overline{\mathbb{N}}_{*}^{j}} \bar{\gamma}_{j}(y)<\infty$. Portanto, para $n$ suficientemente grande, o componente direito de (5.52) é menor que $\varepsilon$ vezes uma constante. O passo subsequente é cuidar de $\sum_{x_{1}=1}^{m_{1}} \ldots \sum_{x_{k}=1}^{m_{k}} \mathcal{G}_{k}^{(n)}(y) p_{y, x}^{(n)}(t)$. Seja

$$
D\left(X_{k}\right) \equiv\left\{t \geq 0 ; \mathbb{P}\left(X_{k}(t)=X_{k}(t-)\right)=1\right\}
$$

Como implicação do Lema A.1 concluimos que o complemento $D^{c}\left(X_{k}\right)$ é no máximo enumerável. Pelo Teorema 3.7.8 de [10], concluimos que

$$
X_{k}^{(n)}(t) \Rightarrow X_{k}(t) \text { para quase todo } t>0
$$

Observe agora que, fixado $t>0, X_{k}^{(n)}(t), X_{k}(t)$ são elementos aleatórios de $\left(\overline{\mathbb{N}}_{*}^{k}, d\right)$, onde $d$ é dado por (5.1), e que uma função $f: \overline{\mathbb{N}}_{*}^{k} \rightarrow \mathbb{R}$ é contínua se e só se, para $x \in \overline{\mathbb{N}}_{*}^{k}$ com $x_{i_{1}}=x_{i_{2}}=\ldots=x_{i_{p}}=\infty, 1 \leq p \leq k$,

$$
f(x)=\lim _{y_{i_{1}}, \ldots, y_{i_{p}} \rightarrow \infty} f(y)
$$

onde $x_{j}=y_{j}$ para $j \in\{1, \ldots, k\} \backslash\left\{i_{1}, \ldots, i_{k}\right\}$. Para $x \in \mathbb{N}_{*}^{k}$, a função $g(z)=\mathbb{I}_{x}(z)$ é limitada e contínua, então

$$
p_{y, x}^{(n)}(t)=\mathbb{E}_{y}\left(g\left(X_{k}^{(n)}(t)\right) \rightarrow \mathbb{E}_{y}\left(g\left(X_{k}(t)\right)\right)=p_{y, x}(t)\right.
$$

quando $n \rightarrow \infty$, onde $\mathbb{E}_{y}(\cdot)$ significa $\mathbb{E}\left(\cdot \mid X_{k}(0)=y\right)$. Consequentemente, para quase todo $t>0$ temos

$$
\sum_{y_{1}=1}^{m_{1}} \ldots \sum_{y_{k}=1}^{m_{k}} \mathcal{G}_{k}^{(n)}(y) p_{y, x}^{(n)}(t) \rightarrow \sum_{y_{1}=1}^{m_{1}} \ldots \sum_{y_{k}=1}^{m_{k}} \mathcal{G}_{k}(y) p_{y, x}(t) \text { quando } n \rightarrow \infty .
$$


Devido à continuidade de $p_{y, x}(\cdot)$, a convergência em (5.57) vale para todo $t>0$, e isso finda a prova de que

$$
\mathcal{G}_{k}(x)=\sum_{y \in \overline{\mathbb{N}}_{*}^{k}} \mathcal{G}_{k}(y) p_{y, x}(t) \text { para todo } t>0
$$

Na Proposição 5.1 provamos que o processo K tem uma medida invariante, porém ainda resta-nos mostrar que ela é única, o que é o tópico da próxima proposição.

Proposição 5.2. O processo K numa árvore tem uma única medida invariante.

Demonstração. Utilizamos aqui a mesma abordagem usada para demonstrar a Proposição 4.12 em [18]. A ideia é discretizar o tempo. Inicialmente classificamos os estados. Dados $h>0$ e $n \in \mathbb{N}_{*}$, tratemos da cadeia de Markov a tempo discreto $Y_{n}^{h}=X_{k}(n h)$. Para $x \in \overline{\mathbb{N}}_{*}^{k} \backslash \mathbb{N}_{*}^{k}$, o Lema 4.3 e o Lema 5.4 implicam $\mathbb{P}\left(X_{k}(n h)=x\right)=0$ para todo $n>0$, que por sua vez implica que $x$ é um estado transiente.

Para $x \in \mathbb{N}_{*}^{k}$, seguimos o mesmo argumento no ínicio da prova do Lema 5.1 para concluir que para cada realização de $X_{k}$ o conjunto $F(x)=\left\{s \in \mathcal{S}_{k} ; s=\sigma_{i}^{\left(x_{k}, k\right)}, i \geq 1\right.$, e $X_{k-1}(s)=$ $\left.\left.x\right|_{k-1}\right\}$ é tal que $|F(x)|=\infty$. Pelo lema de Borel-Cantelli resulta que que $\gamma_{k}(x) T_{s}^{(k)}>h$ infinitas vezes para $s \in F(x)$. Isso e o Lmma 4.1 implicam que $Y_{n}^{h}=x$ para algum $n$, $\operatorname{logo}$ $x$ é recorrente.

Dado que $\mathbb{N}_{*}^{k}$ forma uma classe fechada e irredutível de estados, para cada $h>0, Y_{n}^{h}$ tem uma única medida invariante $\mu^{(h)}$. Já que $\mu^{(h)}=\mu^{(h)} \mathbb{P}^{m}$ para cada $m \in \mathbb{N}_{*}$, concluimos que $\mu^{(h)}=\mu^{(m h)}$, o que prontamente implica que $\mu^{(1)}=\mu^{(h)}$ para todo $h>0$ racional. A fim de obter $\mu^{(1)}=\mu^{(h)}$ pra todo $h>0$, para cada $h>0$ tome uma sequência $\left(h_{p}\right)$ de números racionais tal que $h_{p} \rightarrow h$ as $p \rightarrow \infty$. Então, pelo teorema da convergência dominada e pela continuidade de $p_{y, x}(\cdot)$ temos

$$
\begin{aligned}
\mu^{(1)}(x) & =\sum_{y \in \overline{\mathbb{N}}_{*}^{k}} \mu^{(h)}(y) \mathbb{P}_{y}\left(Y_{1}^{h_{p}}=x\right)=\sum_{y \in \overline{\mathbb{N}}_{*}} \mu^{(h)}(y) p_{y, x}\left(h_{p}\right) \\
& \rightarrow \sum_{y \in \overline{\mathbb{N}}_{*}^{k}} \mu^{(h)}(y) p_{y, x}(h)=\sum_{y \in \overline{\mathbb{N}}_{*}^{k}} \mu^{(h)}(y) \mathbb{P}_{y}\left(Y_{1}^{h}=x\right)
\end{aligned}
$$

quando $p \rightarrow \infty$, e afirmação segue. 


\section{Aplicação ao modelo de armadilhas do GREM}

Neste capítulo aplicamos o Teorema 5.1 para obter um limite de escala do modelo de armadilhas do GREM. Primeiramente definimos esse processo.

\subsection{Modelo de armadilhas do GREM}

Para $j=1, \ldots, k$, seja $\Sigma_{j}:=\left\{\tau_{j}\left(\left.x\right|_{j}\right) ;\left.\left.x\right|_{j} \in \mathcal{M}\right|_{j} ^{(n)}\right\}$ uma sequência de famílias independentes i.i.d. de variáveis aleatórias na bacia de atração duma lei $\alpha_{j}$-estável (veja definição D.3 e (D.4) para mais detalhes) num espaço de probabilidade $(\Omega, \mathcal{F}, P)$, isto é,

$$
P\left(\tau_{j}\left(\left.x\right|_{j}\right)>t\right)=\frac{L_{j}(t)}{t^{\alpha_{j}}}
$$

onde $L_{j}$ é uma função de variação lenta no infinito, ou seja,

$$
\lim _{t \rightarrow \infty} \frac{L_{j}(a t)}{L_{j}(t)}=1 \text { para cada } a>0 .
$$

Supomos

$$
0<\alpha_{1}<\alpha_{2}<\ldots<\alpha_{k-1}<\alpha_{k}<1 \text {. }
$$

Assumiremos de agora em diante que

$$
M_{1}^{(n)}=n
$$

e para $j=1, \ldots, k, n \geq 1$, seja

$$
c_{j}^{(n)}=\left(\inf \left\{t \geq 0: P\left(\tau_{j}\left(\left.x\right|_{j}\right)>t\right) \leq\left(M_{j}^{(n)}\right)^{-1}\right\}\right)^{-1}
$$

$\mathrm{e}$

$$
M_{j+1}^{(n)}=\left(c_{j}^{(n)}\right)^{-1} .
$$

Para cada $j$, rerrotularemos $\Sigma_{j}$, assim obtendo $\Sigma_{j}^{(n)}=\left\{\tau_{j}^{(n)}\left(\left.x\right|_{j}\right) ;\left.\left.x\right|_{j} \in \mathcal{M}\right|_{j} ^{(n)}\right\}$, de modo que $\left\{\tau_{j}^{(n)}\left(\left.x\right|_{j}\right) ; x_{j} \in \mathcal{M}_{j}^{(n)}\right\}$ é estatística de ordem decrescente de $\left\{\tau_{j}\left(\left.x\right|_{j}\right) ; x_{j} \in \mathcal{M}_{j}^{(n)}\right\}$ para cada $\left.\left.x\right|_{j-1} \in \mathcal{M}\right|_{j-1} ^{(n)}$ e $j=1, \ldots, k$ fixado.

Para $j=1, \ldots, k$ e $\left.x\right|_{j} \in V_{k}^{(n)}$, sejam

$$
\gamma_{j}^{(n)}\left(\left.x\right|_{j}\right)=c_{j}^{(n)} \tau_{j}^{(n)}\left(\left.x\right|_{j}\right)
$$

as variáveis aleatórias reescaladas e suponha que a relação (3.9) valha. 
Observação 6.1. Pode-se diretamente verificar do Lema 3.1 que, em termos da descrição de lançamento de moedas, $X_{k}^{(n)} \sim T M_{k}\left(\mathbb{T}_{k}^{(n)} ; \tau_{k} ;\left(p_{j}^{(n)}\right)_{j=1}^{k-1}\right)$, onde para $j=1, \ldots, k-1$ e $\left.\left.x\right|_{j} \in \mathcal{M}\right|_{j}$

$$
p_{j}^{(n)}\left(\left.x\right|_{j}\right)=\frac{1}{1+\tau_{j}^{(n)}\left(\left.x\right|_{j}\right)} .
$$

Definição 6.1. Com essa descrição e valores finitos gerais $M_{1}, \ldots, M_{k}$ (não necessariamente satisfazendo (6.6)), chamamos o modelo de armadilhas $X_{k}^{(n)}$ em $\mathbb{T}_{k}^{(n)}$ com conjunto de parâmetros aleatório $\underline{\gamma_{k}^{(n)}}=\left\{\gamma_{j}^{(n)} ; j=1, \ldots, k\right\}$ o modelo de armadilhas do GREM.

\subsection{Processo $\mathrm{K}$ em $\mathbb{N}_{*}^{k}$ em ambiente aleatório}

Antes de apresentarmos o processo $\mathrm{K}$ em $\mathbb{N}_{*}^{k}$ em ambiente aleatório, precisamos de introduzir um tópico. O objeto a seguir, chamado de cascata de Poisson, fornece-nos o conjunto de parâmetros aleatório de que precisamos para definir nosso processo estocástico.

\subsubsection{Cascata de Poisson}

Aqui apresentamos a cascata de Poisson seguindo [2]. Sua definição é dada em termos de processos pontuais, e o leitor encontrará uma breve introdução ao assunto e alguns resultados no apêndice B.

Seja $k>1$ um inteiro e sejam $0<\alpha_{1}<\ldots<\alpha_{k}<1$ números reais. Relembre a notação $\left.x\right|_{0} \equiv \emptyset \mathrm{e}$, para $j=1, \ldots, k$, seja $\left\{\Delta_{j,\left.x\right|_{j-1}},\left.x\right|_{j-1} \in \mathbb{N}_{*}^{j-1}\right\}$ uma sequência de famílias independentes de processos pontuais de Poisson em $(0, \infty)$ definidas no espaço de probabilidade $(\Omega, \mathcal{F}, P)$ tal que:

i) para $j \in\{1, \ldots, k\},\left.x\right|_{j-1} \in \mathbb{N}_{*}^{j-1}$, a medida de intensidade de $\Delta_{j,\left.x\right|_{j-1}}$ é dada por $\mu_{j}(d t)=\alpha_{j} t^{-1-\alpha_{j}} d t$, e seus pontos são denotados por $\gamma_{j}\left(\left.x\right|_{j}\right)$ e postos em ordem decrescente. Em outras palavras

$$
\Delta_{j,\left.x\right|_{j-1}}=\sum_{x_{j}=1}^{\infty} \mathbb{1}_{\left\{\gamma_{j}\left(\left.x\right|_{j-1}, x_{j}\right)\right\}}
$$

ii) para $j \in\{2, \ldots, k\}$ e $\left.x\right|_{j-2} \in \mathbb{N}_{*}^{j-2}$ fixados, os processos pontuais $\left(\Delta_{j,\left.x\right|_{j-1}}, x_{j-1} \in \mathbb{N}_{*}\right)$ são independentes. 
Seja

$$
\mathcal{P}_{k}=\sum_{x_{1}, \ldots, x_{k}} \mathbb{1}_{\left\{\gamma_{1}\left(\left.x\right|_{1}\right)\right\}} \mathbb{1}_{\left\{\gamma_{2}\left(\left.x\right|_{2}\right)\right\}} \ldots \mathbb{1}_{\left\{\gamma_{k}\left(\left.x\right|_{k}\right)\right\}}
$$

O processo pontual definido por $\mathcal{P}_{k}$ é chamado de cascata de Poisson com $k$ níveis e parâmetros $\alpha_{1}, \alpha_{2}, \ldots, \alpha_{k}$.

\subsubsection{Processo $\mathrm{K}$ em $\mathbb{N}_{*}^{k}$ com parâmetros aleatórios}

Os pontos aleatórios $\gamma_{j}\left(\left.x\right|_{j}\right),\left.x\right|_{j} \in \mathbb{N}_{*}^{j}, j=1, \ldots, k$, que vêm da cascata de Poisson formam conjunto de parâmetros aleatórios que é aplicado à definição que se segue

Definição 6.2. Chamamos $X_{k}$ em $\mathbb{T}_{k}$ com conjunto de parâmetros aleatórios $\underline{\gamma_{k}}=\left\{\gamma_{j} ; j=\right.$ $1, \ldots, k\}$ o processo $K$ com $k$ níveis.

A fim de mostrar que o processo $\mathrm{K} X_{k}$ está bem definido, provemos que a condição (4.2) vale quase certamente. Recorde que

$$
\bar{\gamma}_{k}\left(\left.x\right|_{k}\right)=\prod_{i=1}^{k} \gamma_{i}\left(\left.x\right|_{i}\right)
$$

Queremos provar que

$$
\sum_{x_{1}, \ldots, x_{k}} \bar{\gamma}_{k}\left(\left.x\right|_{k}\right)<\infty \text { a.s. }
$$

Tratemos dos casos $k=1$ e $k=2$. Pelas hipóteses prévias $\Delta_{1, \emptyset}\left(\left.x\right|_{0} \equiv \emptyset\right)$ é um $\operatorname{PPP}\left(\alpha_{1} t^{-1-\alpha_{1}} d t\right)$ e $\Delta_{2, x_{1}}, x_{1} \in \mathbb{N}_{*}$, é um $\operatorname{PPP}\left(\alpha_{2} t^{-1-\alpha_{2}} d t\right)$. Para $a \in\left\{1, \alpha_{2}\right\}$, observamos que

$$
\int_{0}^{\infty}\left(\gamma^{a} \wedge 1\right) d \mu(\gamma)=\int_{0}^{1} \gamma^{a} \alpha_{1} \gamma^{-1-\alpha_{1}} d \gamma+\int_{1}^{\infty} \alpha_{1} \gamma^{-1-\alpha_{1}} d \gamma<\infty
$$

então pelo Teorema B.1

$$
\sum_{x_{1}=1}^{\infty} \gamma_{1}\left(x_{1}\right)^{a}<\infty \text { quase certamente, }
$$

e (6.12) vale para $k=1$.

Ponha $C\left(\alpha_{2}\right)=\sum_{x_{1}=1}^{\infty} \gamma_{1}\left(x_{1}\right)^{\alpha_{2}}$ e defina o processo pontual $\Phi_{2}$ em $(0, \infty)$

$$
\Phi_{2}=\sum_{x_{1}, x_{2}} \mathbb{1}_{\left\{\gamma_{1}\left(x_{1}\right) \gamma_{2}\left(\left.x\right|_{2}\right) / C\left(\alpha_{2}\right)^{\left.1 / \alpha_{2}\right\}}\right.},
$$


e tome $f:(0, \infty) \rightarrow(0, \infty)$ mensurável. Então o funcional de Laplace de $\Phi_{2}$ (veja (B.5)) é dado por

$$
\begin{aligned}
& \psi_{\Phi_{2}}(f)=E \exp \left\{-\sum_{x_{1}, x_{2}} f\left(\frac{\gamma_{1}\left(x_{1}\right) \gamma_{2}\left(\left.x\right|_{2}\right)}{C\left(\alpha_{2}\right)^{1 / \alpha_{2}}}\right)\right\} \\
& =E_{1}\left\{\prod_{x_{1}} E_{2} \exp \left\{-\sum_{x_{2}} f\left(\frac{\gamma_{1}\left(x_{1}\right) \gamma_{2}\left(\left.x\right|_{2}\right)}{C\left(\alpha_{2}\right)^{1 / \alpha_{2}}}\right)\right\}\right\} \\
& =E_{1}\left\{\prod_{x_{1}} \exp \left\{-\alpha_{2} \int_{0}^{\infty}\left(1-\exp \left[-f\left(\frac{\gamma_{1}\left(x_{1}\right) t}{C\left(\alpha_{2}\right)^{1 / \alpha_{2}}}\right)\right]\right) t^{-\alpha_{2}-1} d t\right\}\right\} \\
& =E_{1}\left\{\prod_{x_{1}} \exp \left\{-\frac{\gamma_{1}\left(x_{1}\right)^{\alpha_{2}}}{C\left(\alpha_{2}\right)} \alpha_{2} \int_{0}^{\infty}(1-\exp [-f(t)]) t^{-\alpha_{2}-1} d t\right\}\right\} \\
& =E_{1}\left\{\exp \left\{\sum_{x_{1}}-\frac{\gamma_{1}\left(x_{1}\right)^{\alpha_{2}}}{C\left(\alpha_{2}\right)} \alpha_{2} \int_{0}^{\infty}(1-\exp [-f(t)]) t^{-\alpha_{2}-1} d t\right\}\right\} \\
& =\exp \left\{\alpha_{2} \int_{0}^{\infty}\left(1-e^{-f(t)}\right) t^{-\alpha_{2}-1} d t\right\} \text {, }
\end{aligned}
$$

onde $E_{i}, i=1,2$, é a esperança com respeito aos pontos aleatórios $\gamma_{i}\left(x_{i}\right)$. Portanto, pela Proposição B.3, $\Phi_{2}$ é um $\operatorname{PPP}\left(\alpha_{2} t^{-\alpha_{2}-1} d t\right)$ e

$$
\sum_{x_{1}, x_{2}} \gamma_{1}\left(x_{1}\right) \gamma_{2}\left(\left.x\right|_{2}\right)<\infty \text { q.c. }
$$

O caso geral $k$ segue por indução. Supomos que

$$
C\left(\alpha_{k}\right)=\sum_{x_{1}, \ldots, x_{k-1}} \bar{\gamma}_{k-1}\left(\left.x\right|_{k-1}\right)^{\alpha_{k}}<\infty \text { q.c. }
$$

e consideramos $\Phi_{k}$ o seguinte processo pontual

$$
\Phi_{k}=\sum_{x_{1}, \ldots, x_{k}} \mathbb{1}_{\left\{\gamma_{1}\left(x_{1}\right) \gamma_{2}\left(\left.x\right|_{2}\right) \ldots \gamma_{k}\left(\left.x\right|_{k}\right) / C\left(\alpha_{k}\right)^{\left.1 / \alpha_{k}\right\}}\right.} .
$$

Então fazemos o mesmo cálculo como em (6.16) com $\gamma_{k}\left(x_{k}\right)$ em vez de $\gamma_{2}\left(x_{2}\right)$ e $\bar{\gamma}_{k-1}\left(\left.x\right|_{k-1}\right)$ em vez de $\gamma_{1}\left(x_{1}\right)$, o que implica que $\Phi_{k}$ é um $\operatorname{PPP}\left(\alpha_{k} t^{-\alpha_{k}-1} d t\right)$ e $(6.12)$ vale.

\subsection{Convergência fraca de processos pontuais}

Em seguida nos voltamos para as famílias $\Sigma_{j}^{(n)}:=\left\{\tau_{j}\left(\left.x\right|_{j}\right) ;\left.\left.x\right|_{j} \in \mathcal{M}\right|_{j} ^{(n)}\right\}$ de variáveis aleatórias da Seção 6.1 e construimos os processos pontuais a seguir

$$
\Delta_{j,\left.x\right|_{j-1}}^{(n)} \equiv \sum_{x_{j}=1}^{M_{j}^{(n)}} \mathbb{1}_{\left\{c_{j}^{(n)} \tau_{j}^{(n)}\left(\left.x\right|_{j}\right)\right\}} .
$$


Com (6.9) em mente, verifiquemos que $\Delta_{j,\left.x\right|_{j-1}}^{(n)} \Rightarrow \Delta_{j,\left.x\right|_{j-1}}$ quando $n \rightarrow \infty$ para cada $\left.x\right|_{j-1} \in$ $V_{k}^{(n)}$ fixo, onde $\Delta_{j,\left.x\right|_{j-1}}$ são processos pontuais de Poisson da Seção 6.2.1. Para fazê-lo usamos o fato de que $M_{j}^{(n)} \rightarrow \infty$, cuja prova encontra-se no começo da Seção 6.4. Primeiramente mostremos que

$$
M_{j}^{(n)} P\left[\tau_{j}\left(\left.x\right|_{j}\right)>M_{j+1}^{(n)}\right] \rightarrow 1 \text { as } n \rightarrow \infty .
$$

Pela definição $(6.25)$ de $M_{j}^{(n)} \operatorname{temos} M_{j}^{(n)} P\left[\tau_{j}\left(\left.x\right|_{j}\right)>M_{j+1}^{(n)}\right] \leq 1$. Dado $\varepsilon>0$, então

$$
\begin{aligned}
& P\left[\tau_{j}\left(\left.x\right|_{j}\right)>(1+2 \varepsilon) M_{j+1}^{(n)} /(1+\varepsilon)\right] \leq P\left[\tau_{j}\left(\left.x\right|_{j}\right)>M_{j+1}^{(n)}\right] \\
& \text { and } P\left[\tau_{j}\left(\left.x\right|_{j}\right)>M_{j+1}^{(n)} /(1+\varepsilon)\right] \geq 1 / M_{j}^{(n)}
\end{aligned}
$$

Essas desigualdade e (6.2) implicam

$$
\begin{aligned}
(1+2 \varepsilon)^{-\alpha_{j}} & =\lim _{n \rightarrow \infty} \frac{P\left[\tau_{j}\left(\left.x\right|_{j}\right)>(1+2 \varepsilon) M_{j+1}^{(n)} /(1+\varepsilon)\right]}{P\left[\tau_{j}\left(\left.x\right|_{j}\right)>M_{j+1}^{(n)} /(1+\varepsilon)\right]} \\
& \leq \liminf _{n \rightarrow \infty} \frac{P\left[\tau_{j}\left(\left.x\right|_{j}\right)>M_{j+1}^{(n)}\right]}{1 / M_{j}^{(n)}} .
\end{aligned}
$$

Já que $\varepsilon$ é arbitrário, então (6.21) vale. Usando (6.2) de novo obtemos

$$
M_{j}^{(n)} P\left[\tau_{j}\left(\left.x\right|_{j}\right)>t M_{j+1}^{(n)}\right] \rightarrow t^{-\alpha_{j}}=\mu_{j}((t, \infty)) \text { as } n \rightarrow \infty
$$

que é a medida de intensidade de $\Delta_{j,\left.x\right|_{j-1}}$. Seguindo (6.23) Proposição B.5 afirma que $M_{j}^{(n)} P\left[\tau_{j}\left(\left.x\right|_{j}\right) \in \cdot\right] \stackrel{v}{\rightarrow} \mu_{j}$, o que equivale a dizer que

$$
\Delta_{j,\left.x\right|_{j-1}}^{(n)} \Rightarrow \Delta_{j,\left.x\right|_{j-1}} \text { quando } n \rightarrow \infty
$$

pela Proposição B.8.

Observação 6.2. Com efeito, como se pode ver na prova, a convergência fraca (6.24) não depende da estatística de ordem dos pontos aleatórios de nossos processos pontuais. Esse é somente um modo conveniente de representar os processos pontuais para o acoplamento que será introduzido no próximo capítulo.

\subsection{Verificação das condições (5.2-5.5)}

Nesta subseção conferimos a validade das condições (5.2-5.5) para o modelo de armadilhas do GREM. 
Começamos cuidando de (5.2), a mais fácil e curta condição de verifar. Por hipótese, $M_{1}^{(n)}=n$ e para $j=1, \ldots, k$

$$
M_{j+1}^{(n)}=\inf \left\{t \geq 0: P\left[\tau_{j}\left(\left.x\right|_{j}\right)>t\right] \leq\left(M_{j}^{(n)}\right)^{-1}\right\} .
$$

Suponha que $M_{j}^{(n)} \rightarrow \infty$ quando $n \rightarrow \infty$. Como $P\left[\tau_{j}\left(\left.x\right|_{j}\right)>t\right]>0$ para cada $t>0$, então $M_{j+1}^{(n)} \rightarrow \infty$ quando $n \rightarrow \infty$ e a condição(5.2) vale.

Dado que a convergência em lei como em (6.24) não basta para aplicarmos Teorema 5.1, verificaremos que as condições (5.3-5.5) valem quase certamente para uma representação do ambiente aleatório, isto é, acoplaremos $\underline{\gamma_{k}^{(n)}}$ e $\underline{\gamma_{k}}$ de tal modo que obteremos a convergência forte desejada.

O acoplamento que segue vem de [17], e [14] usa-o para provar a Proposição 6.1 com $k=1$. Aqui estendemos o resultado para $k>1$. Para $t \geq 0$, ponha

$$
G_{j}(t)=P\left(\tau_{j}\left(\left.x\right|_{j}\right)>t\right)=\frac{L_{j}(t)}{t^{\alpha_{j}}} .
$$

$G_{j}$ é não-crescente e contínua à direita. Sabemos por $(6.21)$ que $M_{j}^{(n)} G_{j}\left(\left(c_{j}^{(n)}\right)^{-1}\right) \sim 1$. Seja

$$
G_{j}^{-1}(u)=\inf \left\{t \geq 0: G_{j}(t) \leq u\right\} .
$$

$G_{j}^{-1}$ é não-crescente e contínua à direita. Seja

$$
g_{j}^{(n)}(x)=c_{j}^{(n)} G_{j}^{-1}\left(x / M_{j}^{(n)}\right) .
$$

Sejam $E_{j}\left(\left.x\right|_{j}\right),\left.x\right|_{j} \in \mathbb{N}_{*}^{j}, j=1, \ldots, k$ variáveis aleatórias exponenciais independentes de média 1 num espaço de probabilidade comum $\left(\Omega^{E}, \mathcal{F}^{E}, \mathbf{P}\right)$. Seja

$$
S_{j}\left(\left.x\right|_{j}\right)=\sum_{i=1}^{x_{j}} E_{j}\left(\left.x\right|_{j-1}, i\right)
$$

e seja

$$
\begin{aligned}
& \hat{\gamma}_{j}^{(n)}\left(\left.x\right|_{j}\right)=c_{j}^{(n)} G_{j}^{-1}\left(\frac{S_{j}\left(\left.x\right|_{j}\right)}{S_{j}\left(\left.x\right|_{j-1}, M_{j}^{(n)}\right)}\right), \\
& \hat{\gamma}_{j}\left(\left.x\right|_{j}\right)=S_{j}\left(\left.x\right|_{j}\right)^{-1 / \alpha_{j}} .
\end{aligned}
$$

Provamos agora que $\hat{\gamma}_{j}^{(n)}\left(\left.x\right|_{j}\right)$ e $\gamma_{j}^{(n)}\left(\left.x\right|_{j}\right)$ têm a mesma distribuição, e que $\hat{\gamma}_{j}\left(\left.x\right|_{j}\right)$ e $\gamma_{j}\left(\left.x\right|_{j}\right)$ têm a mesma distribuição. 
Lema 6.1. Para cada $n \geq 1$ e $\left.x\right|_{j-1} \in \mathbb{N}_{*}^{j-1}$, $\left(\hat{\gamma}_{j}^{(n)}\left(\left.x\right|_{j-1}, 1\right), \ldots, \hat{\gamma}_{j}^{(n)}\left(\left.x\right|_{j-1}, x_{j}\right)\right) \stackrel{d}{=}\left(\gamma_{j}^{(n)}\left(\left.x\right|_{j-1}, 1\right), \ldots, \gamma_{j}^{(n)}\left(\left.x\right|_{j-1}, x_{j}\right)\right)$.

Demonstração. Provemos o enunciado para $k=1$. Para $k>1$ a prova é análoga. É sabido que se $U$ é um variável aleatória uniformemente distribuída em $(0,1)$ podemos escrever $\tau_{j}\left(\left.x\right|_{j}\right) \stackrel{d}{=} G_{j}^{-1}(U)$ (veja por exemplo [19], página 4). Por outra lado, é sabido (veja [11], Seção III.3) que se $(U(k), 1 \leq k \leq n)$ são variáveis aleatórias uniformes independentes em $[0,1]$, então denotando por $\bar{U}_{n}(1) \leq \ldots \leq \bar{U}_{n}(n)$ sua estatística de ordem, $\left(\bar{U}_{n}(1), \ldots, \bar{U}_{n}(n)\right) \stackrel{d}{=}$ $\left(S_{1}(1) / S_{1}(n+1), \ldots, S_{1}(n) / S_{1}(n+1)\right)$. A combinação desses dois fatos implica a afirmação.

Em seguida seja $\Upsilon_{j,\left.x\right|_{j-1}}$ o processo pontual em $M_{p}((0, \infty)$ ) (veja o parágrafo logo após (B.2)) que função de contagem

$$
\Upsilon_{j,\left.x\right|_{j-1}}([a, b])=\sum_{i=1}^{\infty} \mathbb{I}_{\hat{\gamma}_{j}\left(\left.x\right|_{j-1}, i\right)}([a, b]) .
$$

Lema 6.2. $\Upsilon_{j,\left.x\right|_{j-1}}$ é um processo pontual de Poisson em $(0, \infty)$ com medida de intensidade $\mu_{j}$.

Demonstração. O processo pontual $\Xi_{j,\left.x\right|_{j-1}}=\sum_{i=1}^{\infty} \mathbb{1}_{S_{j}\left(\left.x\right|_{j-1}, i\right)}$ define um processo pontual de Poisson homogêneo em $(0, \infty)$ e, pela Proposição B.4 para a função $T(t)=t^{-1 / \alpha_{j}}, t>0$, $\Upsilon_{j,\left.x\right|_{j-1}}=\sum_{i=1}^{\infty} \mathbb{1}_{T\left(S_{j}\left(\left.x\right|_{j-1}, i\right)\right)}$ é um processo pontual de Poisson em $(0, \infty)$ com medida de intensidade $\mu_{j}((t, \infty))=T^{-1}(t)=t^{-\alpha_{j}}$.

Recorde que, para $j=1, \ldots, k, \mu_{j}((t, \infty))=t^{-\alpha_{j}} \mathrm{e}$

$$
\int_{0}^{\infty}(u \wedge 1) d \mu_{j}(u)<\infty
$$

Agora estabeleçamos o resultado primcipal desta seção.

Proposição 6.1. Para $k \geq 1$, temos que $\mathbf{P}$-quase certamente

$$
\lim _{n \rightarrow \infty} \sum_{x_{1}=1}^{n} \ldots \sum_{x_{k}=1}^{M_{k}^{(n)}} \hat{\gamma}_{1}^{(n)}\left(\left.x\right|_{1}\right) \ldots \hat{\gamma}_{k}^{(n)}\left(\left.x\right|_{k}\right)=\sum_{x_{1}=1}^{\infty} \ldots \sum_{x_{k}=1}^{\infty} \hat{\gamma}_{1}\left(\left.x\right|_{1}\right) \ldots \hat{\gamma}_{k}^{(n)}\left(\left.x\right|_{k}\right)
$$

As provas da Proposição 6.1 depende fortemente dos próximos dois lemas.

Lema 6.3. Para qualquer $x<\infty$ fixado, $g_{j}^{(n)}(x) \rightarrow x^{-1 / \alpha_{j}}$ quando $n \rightarrow \infty$. 
Demonstração. Observe que $G_{j} \in R_{-\alpha}$ (veja (6.26) e definição C.1). Pelo Lema C.2, $G_{j}^{-1} \in$ $R_{-1 / \alpha}(0+)$, ou seja,

$$
G_{j}^{-1}(x)=\frac{l_{j}(x)}{x^{1 / \alpha_{j}}}
$$

onde $l_{j}$ é função de variação lenta em $0+$ (veja o parágrafo logo depois da definição C.1). Por (6.25) e (6.27), notamos que $M_{j+1}^{(n)}=G_{j}^{-1}\left(1 / M_{j}^{(n)}\right)$. Relembrando (6.6), obtemos

$$
g_{j}^{(n)}(x)=\frac{G_{j}^{-1}\left(x / M_{j}^{(n)}\right)}{G_{j}^{-1}\left(1 / M_{j}^{(n)}\right)}\left[c_{j}^{(n)} G^{-1}\left(1 / M_{j}^{(n)}\right)\right]=x^{-1 / \alpha_{j}} \frac{l_{j}\left(x / M_{j}^{(n)}\right)}{l_{j}\left(1 / M_{j}^{(n)}\right)} .
$$

Como $\frac{l_{j}\left(x / M_{j}^{(n)}\right)}{l_{j}\left(1 / M_{j}^{(n)}\right)} \rightarrow 1$, então $g_{j}^{(n)}(x) \rightarrow x^{-1 / \alpha_{j}}$ quando $n \rightarrow \infty$.

Lema 6.4. Para qualquer $\eta_{j}>0$ fixado, existem constantes $0<C_{j}^{\prime}, C_{j}^{\prime \prime}<\infty$ tais que

$$
g_{j}^{(n)}(x) \leq C_{j}^{\prime} x^{-\left(1-\eta_{j}\right) / \alpha_{j}}, \quad \text { if } \quad C_{j}^{\prime \prime} \leq x \leq M_{j}^{(n)}
$$

Demonstração. Seja $\lambda \in(0,1)$ uma constante cujo valor será escolhido depois, e assuma que $1 / \lambda \leq x \leq M_{j}^{(n)}$. Por $(6.36)$

$$
g_{j}^{(n)}(\lambda x)=(\lambda x)^{-1 / \alpha_{j}} \frac{l_{j}\left(\lambda x / M_{j}^{(n)}\right)}{l_{j}\left(1 / M_{j}^{(n)}\right)}
$$

Pelo Teorema C.1 adaptado ao caso de funções que de variação lenta em zero, podemos escrever

$$
l_{j}(z)=L(1 / z)=\kappa(1 / z) \exp \left\{\int_{1}^{1 / z} \frac{\epsilon(y)}{y} d y\right\}
$$

onde $L$ é função de variação lenta em $\infty$, e o quociente no termo direito de (6.38) torna-se

$$
\frac{\kappa_{j}\left(\frac{M_{j}^{(n)}}{\lambda x}\right)}{\kappa_{j}\left(M_{j}^{(n)}\right)} \exp \left\{-\int_{M_{j}^{(n)} /(\lambda x)}^{M_{j}^{(n)}} \frac{\epsilon_{j}(y)}{y} d y\right\} \quad(\lambda x \geq 1),
$$

onde $\kappa_{j}(y) \rightarrow \kappa_{j} \in(0, \infty)$ e $\epsilon_{j}(y) \rightarrow 0$ quando $y \rightarrow \infty$. Seja $\delta^{\prime}=\sup \left\{\left|\epsilon_{j}(y)\right| ; y \geq 1 / \lambda\right\}$, então

$$
\left|\int_{M_{j}^{(n)} /(\lambda x)}^{M_{j}^{(n)}} \frac{\epsilon_{j}(y)}{y} d y\right| \leq \delta^{\prime} \int_{M_{j}^{(n)} /(\lambda x)}^{M_{j}^{(n)}} \frac{1}{y} d y=\delta^{\prime} \log (\lambda x) .
$$


Assim o expoente em (6.40) é limitado por $(\lambda x)^{\delta^{\prime}}$. Dados $\eta_{j}>0$ e $0<\varepsilon<\kappa_{j}$, escolha $\lambda \in(0,1)$ tal que $\delta^{\prime}(\lambda)<\delta_{j} / \alpha_{j}$ e $\kappa_{j}(y) \in\left(\kappa_{j}-\varepsilon, \kappa_{j}+\varepsilon\right)$ para $y \geq 1 / \lambda$. Visto que $M_{j}^{(n)} /(\lambda x) \geq 1 / \lambda$, então

$$
g_{j}^{(n)}(x) \leq g_{j}^{(n)}(\lambda x) \leq(\lambda x)^{-1 / \alpha_{j}} \frac{\kappa_{j}+\varepsilon}{\kappa_{j}-\varepsilon}(\lambda x)^{\eta_{j} / \alpha_{j}}
$$

e (6.37) segue com $C_{j}^{\prime}=\lambda^{-\left(1-\delta_{j}\right) / \alpha_{j}} \frac{\kappa_{j}+\varepsilon}{\kappa_{j}-\varepsilon}$ and $C_{j}^{\prime \prime}=1 / \lambda$.

Prova da Proposição 6.1: Começamos usando uma estimativa de grandes desvios. Como $2>\mathbf{E}\left(E_{j}\left(\left.x\right|_{j-1}\right)\right)=1$, pelo Teorema 5.11.4 de [15]

$$
\mathbf{P}\left(S_{j}\left(\left.x\right|_{j-1}, M_{j}^{(n)}\right) \geq 2 M_{j}^{(n)}\right) \leq e^{M_{j}^{(n)} \varphi(2)},
$$

onde $\varphi(2)=\lim _{n \rightarrow \infty} \frac{1}{n} \mathbf{P}\left(S_{1}(n) \geq n 2\right)<0$.

Para $j=1, \ldots, k$, ponha $A_{j}^{(n)}:=\left\{S_{j}\left(\left.x\right|_{j-1}, M_{j}\right)^{(n)} \leq 2 M_{j}^{(n)}\right.$ para todo $\left.\left.\left.x\right|_{j-1} \in \mathcal{M}\right|_{j} ^{(n)}\right\} \mathrm{e}$ $A^{(n)}=A_{1}^{(n)} \cap A_{2}^{(n)} \cap \ldots \cap A_{k}^{(n)}$. Logo

$$
\mathbf{P}\left(\left(A^{(n)}\right)^{c}\right) \leq \sum_{j=1}^{k} \mathbf{P}\left(\left(A_{j}^{(n)}\right)^{c}\right) .
$$

Com a ajuda do Lema C.1 e sob as hipótese (6.3), observe que $M_{j}^{(n)} \geq n$ e existe $a \in(0, \infty)$ tal que $\prod_{i=1}^{k} M_{i}^{(n)} \leq n^{a}$ e assim

$$
\mathbf{P}\left(\left(A_{j}^{(n)}\right)^{c}\right) \leq\left(\prod_{i=1}^{j-1} M_{i}^{(n)}\right) e^{M_{j}^{(n)} \kappa(2)} \leq n^{a} e^{n \kappa(2)}
$$

o que implica $\sum_{n=1}^{\infty} \mathbf{P}\left(\left(A_{j}^{(n)}\right)^{c}\right)<\infty$ para $j=1, \ldots, k$. Consequentemente, $\sum_{n=1}^{\infty} \mathbf{P}\left(\left(A^{(n)}\right)^{c}\right)<\infty$ e pelo lema de Borel-Cantelli

$$
\mathbf{P}\left(\left(A^{(n)}\right)^{c} i . v .\right)=0 .
$$

De agora em diante assumimos que $\omega \in \hat{\Omega}^{E}=\left\{\left(A^{(n)}\right)^{c} i . o .\right\}^{c}$ e usamos o fato de que por (6.46), para $j=1, \ldots, k$

$$
S_{j}\left(\left.x\right|_{j-1}, M_{j}^{(n)}\right) \leq 2 M_{j}^{(n)}
$$

para todo $\left.\left.x\right|_{j-1} \in \mathcal{M}\right|_{j-1} ^{(n)}$ e $n$ bastante grande.

Inicialmente, atacamos (6.34) com $k=1$. Para $y>0$, ponha

$$
I(y)=\left\{x_{1} \geq 1 ; \hat{\gamma}\left(x_{1}\right) \geq y\right\}, \quad I^{c}(y)=\left\{x_{1} \geq 1 ; \hat{\gamma}\left(x_{1}\right)<y\right\} .
$$


Então para $\delta_{1}>0$ temos

$$
\sum_{x_{1}=1}^{n} \hat{\gamma}_{1}^{(n)}\left(x_{1}\right)=\sum_{x_{1} \in I\left(\delta_{1}\right)} \hat{\gamma}_{1}^{(n)}\left(x_{1}\right)+\sum_{x_{1} \in I\left(n^{-1 / \alpha_{1}}\right) \backslash I\left(\delta_{1}\right)} \hat{\gamma}_{1}^{(n)}\left(x_{1}\right)+\sum_{x_{1} \in I^{c}\left(n^{\left.-1 / \alpha_{1}\right)}\right.} \hat{\gamma}_{1}^{(n)}\left(x_{1}\right)
$$

De (6.33) temos $E\left(\left|I\left(\delta_{1}\right)\right|\right)=\mu_{1}\left(\left(\delta_{1}, \infty\right)\right)<\infty$, logo $\left|I\left(\delta_{1}\right)\right|<\infty$ quase certamente. Então segue do Lema 6.3 que

$$
\lim _{n \rightarrow \infty} \sum_{x_{1} \in I\left(\delta_{1}\right)} \hat{\gamma}_{1}^{(n)}\left(x_{1}\right)=\sum_{x_{1} \in I\left(\delta_{1}\right)} \hat{\gamma}_{1}\left(x_{1}\right)
$$

Para $x_{1} \in I\left(n^{-1 / \alpha_{1}}\right) \backslash I\left(\delta_{1}\right)$, note que $\delta_{1}^{-\alpha_{1}}<S_{1}\left(x_{1}\right) \leq n$. Agora para $\eta_{1}>0$, pelo Lema 6.4, existem $C_{1}^{\prime}, C_{1}^{\prime \prime}$ em $(0, \infty)$ tais que $\left(\delta_{1}\right.$ suficientemente pequeno para que $\left.\delta_{1}^{-\alpha_{1}}>C_{1}^{\prime \prime}\right)$

$$
\begin{aligned}
\sum_{x_{1} \in I\left(n^{-1 / \alpha_{1}}\right) \backslash I\left(\delta_{1}\right)} \hat{\gamma}_{1}^{(n)}\left(\left.x\right|_{1}\right) & \leq C_{1}^{\prime} \sum_{x_{1} \in I\left(n^{-1 / \alpha_{1}}\right) \backslash I\left(\delta_{1}\right)} S_{1}\left(\left.x\right|_{1}\right)^{-\left(1-\eta_{1}\right) / \alpha_{1}} \\
& =C_{1}^{\prime} \sum_{x_{1} \in I\left(n^{-1 / \alpha_{1}}\right) \backslash I\left(\delta_{1}\right)} \hat{\gamma}_{1}\left(\left.x\right|_{1}\right)^{\left(1-\eta_{1}\right)} .
\end{aligned}
$$

A derradeira soma em (6.50) é limitada por cima por

$$
W_{\delta_{1}}=\sum_{x_{1} ; \hat{\gamma}_{1}\left(x_{1}\right) \leq \delta_{1}} \hat{\gamma}_{1}\left(x_{1}\right)^{\left(1-\eta_{1}\right)}
$$

Com $\eta_{1}>0$ suficientemente pequeno tal que $\eta_{1}+\alpha_{1}<1$, mostremos que $W:=\lim _{\delta_{1} \rightarrow \infty} W_{\delta_{1}}$ $=0 \mathbf{P}$-quase certamente. Primeiramente observe que $W \geq 0$ and $W_{\delta_{1}}$ decresce quando $\delta_{1}$ decresce, assim $W$ está bem definida. En segundo lugar,

$$
E\left(W_{\delta_{1}}\right)=\int_{0}^{\delta_{1}} t^{1-\eta_{1}} \mu_{1}(d t)=\alpha_{1} \int_{0}^{\delta_{1}} t^{1-\eta_{1}} t^{-1-\alpha_{1}} d t=\frac{\alpha_{1}}{1-\eta_{1}-\alpha_{1}} \delta_{1}^{1-\eta_{1}-\alpha_{1}} .
$$

e então $\lim _{\delta_{1} \rightarrow \infty} E\left(W_{\delta_{1}}\right)=0$. Em seguida aplicamos o teorema da convergência dominada com $g=\sum_{x_{1} ; \hat{\gamma}_{1}\left(x_{1}\right) \leq \nu} \hat{\gamma}_{1}\left(x_{1}\right)^{\left(1-\eta_{1}\right)}$ para algum $\nu \in(0,1)$ fixado e o resultado se segue. Portanto

$$
\limsup _{\delta_{1} \rightarrow 0} \limsup _{n \rightarrow \infty} \sum_{x_{1} \in I\left(n^{-1 / \alpha_{1}}\right) \backslash I\left(\delta_{1}\right)} \hat{\gamma}_{1}^{(n)}\left(x_{1}\right)=0
$$

Para $x_{1} \in I^{c}\left(n^{-1 / \alpha_{1}}\right)$, temos $S_{1}\left(x_{1}\right) / n>1$. Visto que $G_{1}^{-1}$ é não-crescente e por (6.47), $\log 0$

$$
\left.G_{1}^{-1}\left(S_{1}\left(x_{1}\right) / S_{1}(n)\right) \leq G_{1}^{-1}\left(S_{1}\left(x_{1}\right) / 2 n\right)\right) \leq G_{1}^{-1}(1 / 2)
$$


Então

$$
\sum_{x_{1} \in I^{c}\left(n^{\left.-1 / \alpha_{1}\right)}\right.} \hat{\gamma}_{1}^{(n)}\left(x_{1}\right) \leq \sum_{x_{1} \in I^{c}\left(n^{\left.-1 / \alpha_{1}\right)}\right.} c_{1}^{(n)} \leq n c_{1}^{(n)} .
$$

Tomamos da prova do Lema 6.3 que $c_{1}^{(n)} G_{1}^{-1}\left(1 / M_{2}^{(n)}\right)=1$, do Lema C.2 que $G_{1}^{-1} \in R_{-1 / \alpha_{1}}\left(0_{+}\right)$, ou seja, $G^{-1}(x)=l_{1}(x) / x^{1 / \alpha_{1}}$, onde $l_{1}$ é de variação lenta em $0+$. Pelo Lema C.1 adaptado a $l_{1}$, dado $\epsilon>0$, temos

$$
t^{\epsilon}<l_{1}(t)<t^{-\epsilon} \text { para } t \text { suficientemente pequeno. }
$$

Agora pegue $\left(1 / \alpha_{1}\right)-1>\epsilon>0$ e $n$ bastante grande tal que $l_{1}(1 / n)>(1 / n)^{\epsilon}$. Assim

$$
n c_{1}^{(n)}=\frac{n}{G_{1}^{-1}(1 / n)} c_{1}^{(n)} G_{1}^{-1}(1 / n) \sim \frac{n}{l_{1}(1 / n)(1 / n)^{-1 / \alpha_{1}}} \rightarrow 0 \text { as } n \rightarrow \infty .
$$

Portanto

$$
\limsup _{n \rightarrow \infty} \sum_{x_{1} \in I^{c}\left(n^{\left.-1 / \alpha_{1}\right)}\right.} \hat{\gamma}_{1}^{(n)}\left(x_{1}\right)=0
$$

Juntando (6.49), (6.53) e (6.58) obtemos que

$$
\lim _{n \rightarrow \infty} \sum_{x_{1}=1}^{n} \hat{\gamma}_{1}^{(n)}\left(\left.x\right|_{1}\right)=\sum_{x_{1}=1}^{\infty} \hat{\gamma}_{1}\left(\left.x\right|_{1}\right) .
$$

Depois de provarmos (6.34) para $k=1$, demonstraremos o caso geral por indução. Suponha que (6.34) valha para $k-1(k \geq 2)$.

Para $y>0$ e cada $\left.x\right|_{j-1}$, ponha $I_{j,\left.x\right|_{j-1}}(y)=\left\{\left.x\right|_{j} ; \hat{\gamma}\left(\left.x\right|_{j}\right) \geq y\right\}$. Como em (6.48) quebramos cada uma das $k$ somas abaixo

$$
\sum_{x_{1}=1}^{n} \hat{\gamma}_{1}^{(n)}\left(\left.x\right|_{1}\right) \ldots \sum_{x_{k}=1}^{M_{k}^{(n)}} \hat{\gamma}_{k}^{(n)}\left(\left.x\right|_{k}\right)
$$

em 3 partes, de maneira que a $j$-ésima soma se escreve como

$$
\sum_{x_{j}}^{(1)}+\sum_{x_{j}}^{(2)}+\sum_{x_{j}}^{(3)}
$$

onde dado $\delta_{j} \in(0, \infty)$ a primeira soma é sobre $I_{j,\left.x\right|_{j-1}}\left(\delta_{j}\right)$, a segunda é sobre $I_{j,\left.x\right|_{j-1}}\left(\left(M_{j}^{(n)}\right)^{-1 / \alpha_{j}}\right) \backslash I_{j,\left.x\right|_{j-1}}\left(\delta_{j}\right)$ e a terceira é sobre $I_{j,\left.x\right|_{j-1}}^{c}\left(\left(M_{j}^{(n)}\right)^{-1 / \alpha_{j}}\right)$. Segue do Lmma 6.3 que

$$
\sum_{x_{1}}^{(1)} \hat{\gamma}_{1}^{(n)}\left(\left.x\right|_{1}\right) \ldots \sum_{x_{k}}^{(1)} \hat{\gamma}_{k}^{(n)}\left(\left.x\right|_{k}\right) \rightarrow \sum_{x_{1}}^{(1)} \hat{\gamma}_{1}\left(\left.x\right|_{1}\right) \ldots \sum_{x_{k}}^{(1)} \hat{\gamma}_{k}^{(n)}\left(\left.x\right|_{k}\right)
$$


quando $n \rightarrow \infty$ quase certamente, já que essas somas são somas sobre o conjunto $I_{1}\left(\delta_{1}\right) \times$ $I_{2, x_{1}}\left(\delta_{2}\right) \times \ldots \times I_{k,\left.x\right|_{k-1}}\left(\delta_{k}\right)$, que é quase certamente finito pois

$$
\begin{aligned}
E\left(\left|I_{1}\left(\delta_{1}\right)\right|\left|I_{2, x_{1}}\left(\delta_{2}\right)\right| \ldots\left|I_{k, x||_{k-1}}\left(\delta_{j}\right)\right|\right) & =\prod_{i=1}^{k} E\left(\left|I_{i,\left.x\right|_{i-1}}\left(\delta_{i}\right)\right|\right) \\
& =\prod_{i=1}^{k} \mu_{i}\left(\left(\delta_{i}, \infty\right)\right)<\infty
\end{aligned}
$$

onde usamos em (6.63) a independência dos processos de Poisson $\Delta_{j,\left.x\right|_{j-1}}$ (veja a subseção 6.2.1) e o fato de que $\mu_{i}\left(\left(\delta_{i}, \infty\right)\right)=\delta_{i}^{-\alpha_{i}}$. Mostraremos que

$$
\limsup _{\delta_{1}, \ldots, \delta_{k} \rightarrow 0} \limsup _{n \rightarrow \infty} \sum_{x_{1}}^{\left(i_{i}\right)} \hat{\gamma}_{1}^{(n)}\left(\left.x\right|_{1}\right) \ldots \sum_{x_{k}}^{\left(i_{k}\right)} \hat{\gamma}_{k}^{(n)}\left(\left.x\right|_{k}\right)=0
$$

quase certamente para todo $\left(i_{1}, \ldots, i_{k}\right) \in\{1,2,3\}^{k} \backslash\{(1,1, \ldots, 1)\}$. Visto que $\sum_{x_{1}}^{(1)}$ é uma soma sobre um conjunto quase certamente finito, e usando a hipótese de indução, basta considerar as somas

$$
\sum_{x_{1}}^{\left(i_{1}\right)} \hat{\gamma}_{1}^{(n)}\left(\left.x\right|_{1}\right) \ldots \sum_{x_{k}}^{\left(i_{k}\right)} \hat{\gamma}_{k}^{(n)}\left(\left.x\right|_{k}\right)
$$

com $i_{1} \in\{2,3\}$.

Dados $j=2, \ldots, k$ e $\eta_{j}>0$, primeiro consideramos o caso $i_{1}=2$ e $i_{j} \in\{1,2\}$ e mostramos que

$$
\hat{\gamma}_{j}^{(n)}\left(\left.x\right|_{j}\right) \leq C_{j}^{\prime}\left[\left(\hat{\gamma}_{j}\left(\left.x\right|_{j}\right)\right)^{1-\eta_{j}} \vee\left(\hat{\gamma}_{j}\left(\left.x\right|_{j}\right)\right)^{1+\eta_{j}}\right]
$$

para $\operatorname{algum} C_{j}^{\prime}>0$. Se $\left.x\right|_{j} \in I\left(\left(M_{j}^{(n)}\right)^{-1 / \alpha_{j}}\right) \backslash I\left(\delta_{j}\right)$, então $\delta_{j}^{-\alpha_{j}}<S_{j}\left(\left.x\right|_{j}\right)<M_{j}^{(n)}$. Como $G_{j}^{-1}$ é não-crescente e por $(6.28),(6.30),(6.31),(6.47)$ e Lema 6.4 ( $\delta_{j}$ pequeno o suficente), então

$$
\hat{\gamma}_{j}^{(n)}\left(\left.x\right|_{j}\right) \leq g_{j}^{(n)}\left(S_{j}\left(\left.x\right|_{j}\right) / 2\right) \leq C_{j}^{\prime}\left(\hat{\gamma}_{j}\left(\left.x\right|_{j}\right)\right)^{1-\eta_{j}}
$$

Se $\left.x\right|_{j} \in I\left(\delta_{j}\right)$, então $S_{j}\left(\left.x\right|_{j}\right) \leq \delta_{j}^{-\alpha_{j}}$. Como $G_{j}^{-1}$ é não-crescente e por (6.30), (6.28), (6.47) e (6.36), então

$$
\hat{\gamma}_{j}^{(n)}\left(\left.x\right|_{j}\right) \leq g_{j}^{(n)}\left(S_{j}\left(\left.x\right|_{j}\right) / 2\right)=\left(S_{j}\left(\left.x\right|_{j}\right) / 2\right)^{-1 / \alpha_{j}} \frac{l_{j}\left(S_{j}\left(\left.x\right|_{j}\right) / 2 M_{j}^{(n)}\right)}{l_{j}\left(1 / 2 M_{j}^{(n)}\right)}
$$


Posteriormente apelamos ao Teorema C.1 adaptado ao caso de funções que são de variação lenta em zero (veja (6.39)) para obter o limitante superior seguinte para o quociente do membro direito de (6.68)

$$
\frac{\kappa_{j}\left(\frac{2 M_{j}^{(n)}}{S_{j}\left(\left.x\right|_{j}\right)}\right)}{\kappa_{j}\left(2 M_{j}^{(n)}\right)} \exp \left\{\left|\int_{a}^{b} \frac{\epsilon_{j}(y)}{y} d y\right|\right\}
$$

onde $a=2 M_{j}^{(n)} \wedge \frac{2 M_{j}^{(n)}}{S_{j}\left(\left.x\right|_{j}\right)}$ e $b=2 M_{j}^{(n)} \vee \frac{2 M_{j}^{(n)}}{S_{j}\left(\left.x\right|_{j}\right)}$. Tome $n$ bastante grande para que $\delta^{\prime}=$ $\sup \left\{\epsilon_{j}(y) ; y \geq M_{j}^{(n)} \delta_{j}\right\}<\eta_{j} / \alpha_{j}$ e, dado $\epsilon>0\left(0<\epsilon<\kappa_{j}\right), \kappa_{j}(y) \in\left(\kappa_{j}-\epsilon, \kappa_{j}+\epsilon\right)$ for $y \geq M_{j}^{(n)} \delta_{j}$, então

$$
\left|\int_{a}^{b} \frac{\epsilon_{j}(y)}{y} d y\right| \leq \delta^{\prime}\left|\log \left(S_{j}\left(\left.x\right|_{j}\right)\right)\right| \leq \frac{\eta_{j}}{\alpha_{j}}\left|\log \left(S_{j}\left(\left.x\right|_{j}\right)\right)\right| .
$$

Relembrando (6.31), logo

$$
\begin{aligned}
\hat{\gamma}_{j}^{(n)}\left(\left.x\right|_{j}\right) & \leq C_{j}^{\prime} S_{j}\left(\left.x\right|_{j}\right)^{-1 / \alpha_{j}} \exp \left\{\left|\log \left(S_{j}\left(\left.x\right|_{j}\right)^{\eta_{j} / \alpha_{j}}\right)\right|\right\} \\
& =C_{j}^{\prime}\left[\left(\hat{\gamma}_{j}\left(\left.x\right|_{j}\right)\right)^{1-\eta_{j}} \vee\left(\hat{\gamma}_{j}\left(\left.x\right|_{j}\right)\right)^{1+\eta_{j}}\right]
\end{aligned}
$$

e (6.66) vale. Substituimos $\vee$ por + em (6.66) e obtemos um limitante superior para (6.65) em termos de $2^{k-1}$ somas da forma constante vezes

$$
\sum_{x_{1} ; \hat{\gamma}_{1}\left(\left.x\right|_{1}\right) \leq \delta_{1}}\left(\hat{\gamma}_{1}\left(\left.x\right|_{1}\right)\right)^{1-\eta_{1}} \sum_{x_{2}}\left(\hat{\gamma}_{2}\left(\left.x\right|_{2}\right)\right)^{1 \pm \eta_{2}} \ldots \sum_{x_{k}}\left(\hat{\gamma}_{k}\left(\left.x\right|_{k}\right)\right)^{1 \pm \eta_{k}}
$$

A fim de mostrarmos que (6.71) converge quase certamente a 0 quando $\delta_{1} \rightarrow 0$, escolhamos $\eta_{j}, j=1, \ldots, k$, pequeno o suficiente tal que

$$
\frac{\alpha_{1}}{1-\eta_{1}}<\frac{\alpha_{2}}{1 \pm \eta_{2}}<\ldots<\frac{\alpha_{k}}{1 \pm \eta_{k}}<1
$$

Em seguida demonstremos que para cada $x_{1}$

$$
\chi_{k}\left(x_{1}\right):=\sum_{x_{2}}\left(\hat{\gamma}_{2}\left(\left.x\right|_{2}\right)\right)^{1 \pm \eta_{2}} \ldots \sum_{x_{k}}\left(\hat{\gamma}_{k}\left(\left.x\right|_{k}\right)\right)^{1 \pm \eta_{k}}
$$

é uma variável aleatória $\frac{\alpha_{2}}{1 \pm \eta_{2}}$-estável. Primeiro mostramos que

$$
\sum_{x_{j}}\left(\hat{\gamma}_{j}\left(\left.x\right|_{j}\right)\right)^{1 \pm \eta_{j}}
$$


é uma lei $\frac{\alpha_{j}}{1 \pm \eta_{j}}$-estável. Usando a transformada de Laplace (que determina unicamente uma v.a., veja o Teorema XIII.1.1 de [11]) e Proposição (B.3) $\operatorname{com} f(x)=x^{1 \pm \eta_{j}}$, para $\lambda>0$, temos

$$
\begin{aligned}
& E\left\{\exp \left\{-\lambda \sum_{x_{j}}\left(\hat{\gamma}_{j}\left(\left.x\right|_{j}\right)\right)^{1 \pm \eta_{j}}\right\}\right\}=\exp \left\{-\alpha_{j} \int_{0}^{\infty}\left(1-e^{-\lambda t^{1 \pm \eta_{j}}}\right) t^{-\alpha_{j}-1} d t\right\} \\
& =\exp \left\{-\lambda^{\alpha_{j} /\left(1 \pm \eta_{j}\right)} \frac{\alpha_{j}}{1 \pm \eta_{j}} \int_{0}^{\infty}\left(1-e^{-t}\right) t^{-1-\frac{\alpha_{j}}{1 \pm \eta_{j}}} d t\right\}
\end{aligned}
$$

que pela Proposição D.1 é variável aleatória estável com índice $\frac{\alpha_{j}}{1 \pm \eta_{j}}$. Em seguida aplicamos as mesmas ideias a (6.73) com $k=3$. Para $k>3$ a prova é similar. Então

$$
\begin{aligned}
& E\left\{\exp \left\{-\lambda \sum_{x_{2}, x_{3}}\left(\gamma_{2}\left(\left.x\right|_{2}\right)\right)^{1 \pm \eta_{2}}\left(\gamma_{3}\left(\left.x\right|_{3}\right)\right)^{1 \pm \eta_{3}}\right\}\right\} \\
& =E_{2}\left\{\prod_{x_{2}} E_{3} \exp \left\{-\lambda\left(\gamma_{2}\left(\left.x\right|_{2}\right)\right)^{1 \pm \eta_{2}} \sum_{x_{3}}\left(\gamma_{3}\left(\left.x\right|_{3}\right)\right)^{1 \pm \eta_{3}}\right\}\right\} \\
& =E\left\{\exp \left\{-c \lambda^{\alpha_{3} /\left(1 \pm \eta_{3}\right)} \sum_{x_{2}}\left(\gamma_{2}\left(\left.x\right|_{2}\right)\right)^{\frac{\alpha_{3}}{1 \pm \eta_{3}} 1 \pm \eta_{2}}\right\}\right\} \\
& =\exp \left\{-\frac{\alpha_{2}}{1 \pm \eta_{2}} \int_{0}^{\infty}\left(1-e^{-c(\lambda t)^{\alpha_{3} /\left(1 \pm \eta_{3}\right)}}\right) t^{-1-\frac{\alpha_{2}}{1 \pm \eta_{2}}} d t\right\} \\
& =\exp \left\{-\lambda^{\frac{\alpha_{2}}{1 \pm \eta_{2}}} c^{\frac{\alpha_{2}\left(1 \pm \eta_{3}\right)}{\alpha_{3}\left(1 \pm \eta_{2}\right)}} \frac{\alpha_{2}}{1 \pm \eta_{2}} \int_{0}^{\infty}\left(1-e^{-t}\right) t^{-1-\frac{\alpha_{2}\left(1 \pm \eta_{3}\right)}{\alpha_{3}\left(1 \pm \eta_{2}\right)}} d t\right\}
\end{aligned}
$$

onde $c>0$ é uma constante, e a expressão em (6.73) é de fato um variável aleatória estável com índice $\frac{\alpha_{2}}{1 \pm \eta_{2}}$. Com isso em mente e usando (6.73) a transformada de Laplace de (6.71) dá

$$
\begin{aligned}
& E\left\{\exp \left\{-\lambda \sum_{x_{1} ; \hat{\gamma}_{1}\left(\left.x\right|_{1}\right) \leq \delta_{1}}\left(\gamma_{1}\left(\left.x\right|_{1}\right)\right)^{1-\eta_{1}} \chi_{k}\left(x_{1}\right)\right\}\right\} \\
& =E\left\{\exp \left\{-c \lambda^{\alpha_{2} /\left(1 \pm \eta_{2}\right)} \sum_{x_{1} ; \hat{\gamma}_{1}\left(\left.x\right|_{1}\right) \leq \delta_{1}}\left(\gamma_{1}\left(x_{1}\right)\right)^{1-\eta_{1} \frac{\alpha_{2}}{1 \pm \eta_{2}}}\right\}\right\} \\
& =\exp \left\{-\lambda^{\frac{\alpha_{1}}{1-\eta_{1}}} c^{\prime} \int_{0}^{\delta_{1}}\left(1-e^{-t}\right) t^{-1-\frac{\alpha_{1}\left(1 \pm \eta_{2}\right)}{\alpha_{2}\left(1 \pm \eta_{1}\right)}} d t\right\} \quad\left(e^{-t}>1-t \text { for } t \in(0,1)\right) \\
& \geq \exp \left\{-\lambda^{\frac{\alpha_{1}}{1-\eta_{1}}} c^{\prime} \delta_{1}^{1-\frac{\alpha_{1}}{1-\eta_{1}} \frac{1 \pm \eta_{2}}{\alpha_{2}}}\right\} \rightarrow 1 \text { as } \delta_{1} \rightarrow 0,
\end{aligned}
$$

onde $c, c^{\prime}>0$ são constantes e usamos (6.72) para chegar à conclusão. O limite em (6.76) implica que (6.71) converge a a 0 em distribuição quando $\delta_{1} \rightarrow 0$ e, como o limite é uma 
constante, então (6.71) converge a 0 em probabilidade. Para concluir a convergência quase certa de (6.71) tomamos uma subsequência $\left\{\delta_{1, p_{i}}\right\}, i \in \mathbb{N}$, tal que (6.71) converge q.c. a 0 (veja o Teorema 7.2.13 de [15]) e pela monotonicidade a afirmação vale. Assim (6.64) segue para o caso $i_{1}=2$ e $i_{j} \in\{1,2\}$ para todo $j=2, \ldots, k$.

Olhemos para a expressão (6.65) quando $\mathcal{L}:=\left\{j=1, \ldots, k ; i_{j}=3\right\} \neq \emptyset$. Para $\left.x\right|_{j} \in$ $I^{c}\left(\left(M_{j}^{(n)}\right)^{-1 / \alpha_{j}}\right)$, vemos que $S_{j}\left(\left.x\right|_{j}\right) / M_{j}^{(n)}>1$. Já que $G_{j}^{-1}$ é não-crescente, temos

$$
G_{j}^{-1}\left(S_{j}\left(\left.x\right|_{j}\right) / 2 M_{j}^{(n)}\right) \leq G_{j}^{-1}(1 / 2) .
$$

Então, observando (6.30) e por (6.47)

$$
\hat{\gamma}_{j}^{(n)}\left(\left.x\right|_{j}\right) \leq G_{j}^{-1}(1 / 2) c_{j}^{(n)} .
$$

Seja $p=|\{1, \ldots, k\} \backslash \mathcal{L}|$ e seja $1 \leq d_{1}<\ldots<d_{p} \leq k$ uma enumeração de $\{1, \ldots, k\} \backslash \mathcal{L}$. Ao argumentar como acima obtemos um limitante superior para (6.65) em termos de $2^{k-p}$ somas da forma constante vezes

$$
B_{1}^{(n)} B_{2}^{(n)} \ldots B_{p}^{(n)} \prod_{j=d_{p}+1}^{k} c_{j}^{(n)} M_{j}^{(n)}
$$

onde $d_{0} \equiv 0$ and, for $s=1, \ldots, p$,

$$
B_{s}^{(n)}=\prod_{j=d_{s-1}+1}^{d_{s}-1} c_{j}^{(n)} \sum_{x_{d_{s-1}+1}}^{M_{d_{s-1}+1}^{(n)}} \cdots \sum_{x_{d_{s}-1}}^{M_{d_{s}-1}^{(n)}} \sum_{x_{d_{s}}}\left(\hat{\gamma}_{d_{s}}\left(\left.x\right|_{d_{s}}\right)\right)^{1 \pm \eta_{d_{s}}}
$$

Em seguida provemos que a transformada de Laplace de (6.79) converge a 1 quando $n \rightarrow \infty$. Inicialmente relembramos que $c_{j}^{(n)}=\left(M_{j+1}^{(n)}\right)^{-1}$ e então usamos o Lema C.1 para ver que, dado $\epsilon>0$,

$$
\left(M_{j}^{(n)}\right)^{\frac{1}{\alpha_{j}}-\epsilon}<M_{j+1}^{(n)}<\left(M_{j}^{(n)}\right)^{\frac{1}{\alpha_{j}}+\epsilon},
$$

o que implica que, para $\epsilon$ pequeno o suficiente, ambos $c_{j}^{(n)} M_{j}^{(n)}$ e o produto no lado direito de (6.79) decaem polinomialmente a 0 quando $n \rightarrow \infty$. Também observamos que a transformada de Laplace de (6.80) $(\lambda>0)$ é

$$
E\left\{\exp \left\{-\lambda B_{s}^{(n)}\right\}\right\}=\exp \left\{-c^{\prime} \lambda^{\frac{\alpha_{d_{s}}}{1 \pm \eta_{d_{s}}}} \prod_{j=d_{s-1}+1}^{d_{s}-1}\left(c_{j}^{(n)}\right)^{\frac{\alpha_{d_{s}}}{1 \pm \eta_{d_{s}}}} M_{j}^{(n)}\right\},
$$


onde $c^{\prime}>0$ z'e uma constante. Usando (6.81) e a hipótese $\alpha_{d_{s}}>\alpha_{j}$ para $j=d_{s-1}+1, \ldots, d_{s}-$ 1 , concluimos que $\left(c_{j}^{(n)}\right)^{\frac{\alpha_{d_{s}}}{1 \pm \eta_{d_{s}}}} M_{j}^{(n)}$ decae polinomialmente a 0 quando $n \rightarrow \infty$ e assim $(6.82)$ vai a 1 . Suponha agora que

$$
E\left\{\exp \left\{-\lambda B_{1}^{(n)} B_{2}^{(n)} \ldots B_{p}^{(n)} \prod_{j=d_{p}+1}^{k} c_{j}^{(n)} M_{j}^{(n)}\right\}\right\}=\exp \left\{-c^{\prime \prime} \lambda^{\frac{\alpha_{d_{1}}}{1 \pm \eta_{d_{1}}}} b\right\}
$$

onde $c^{\prime \prime}>0$ é uma constante e $b$ é dado por

$$
b=\left(\prod_{j=d_{p}+1}^{k} c_{j}^{(n)} M_{j}^{(n)}\right)^{\frac{\alpha_{d_{1}}}{1 \pm \eta_{d_{1}}}} \prod_{s=1}^{p}\left(\prod_{j=d_{s-1}+1}^{d_{s}-1}\left(c_{j}^{(n)}\right)^{\frac{\alpha_{d_{s}}}{1 \pm \eta_{d_{s}}}} M_{j}^{(n)}\right)^{\frac{\alpha_{d_{1}}}{1 \pm \eta_{d_{1}}} \frac{1 \pm \eta_{d_{s}}}{\alpha_{d_{s}}}} .
$$

Provemos que e expoente (6.84) faz sentido para $p+1$. Primeiramente note que (6.82) estabelece o resultado para $p=1$. Assim

$$
\begin{aligned}
& E\left\{\exp \left\{-\lambda B_{1}^{(n)} B_{2}^{(n)} \ldots B_{p+1}^{(n)} \prod_{j=d_{p+1}+1}^{k} c_{j}^{(n)} M_{j}^{(n)}\right\}\right\} \\
& =E\left\{\prod_{x_{1}, \ldots, x_{d_{1}}} \exp \left\{-\lambda \prod_{j=1}^{d_{1}-1} c_{j}^{(n)}\left(\hat{\gamma}_{d_{1}}\left(\left.x\right|_{d_{1}}\right)\right)^{1 \pm \eta_{d_{1}}} B_{2}^{(n)} \ldots B_{p+1}^{(n)} \prod_{j=d_{p+1}+1}^{k} c_{j}^{(n)} M_{j}^{(n)}\right\}\right\} \\
& =E\left\{\prod_{x_{1}, \ldots, x_{d_{1}}} \exp \left\{-c^{\prime}\left(\lambda \prod_{j=1}^{d_{1}-1} c_{j}^{(n)}\left(\hat{\gamma}_{d_{1}}\left(\left.x\right|_{d_{1}}\right)\right)^{1 \pm \eta_{d_{1}}}\right)^{\frac{\alpha_{d_{2}}}{1 \pm \eta_{d_{2}}}} b^{\prime}\right\}\right\}
\end{aligned}
$$

onde $c^{\prime}>0$ é uma constante e

$$
b^{\prime}=\left(\prod_{j=d_{p+1}+1}^{k} c_{j}^{(n)} M_{j}^{(n)}\right)^{\frac{\alpha_{d_{2}}}{1 \pm \eta_{d_{2}}}} \prod_{s=2}^{p+1}\left(\prod_{j=d_{s-1}+1}^{d_{s}-1}\left(c_{j}^{(n)}\right)^{\frac{\alpha_{d_{s}}}{1 \pm \eta_{d_{s}}}} M_{j}^{(n)}\right)^{\frac{\alpha_{d_{2}}}{1 \pm \eta_{2}}} \frac{1 \pm \eta_{d_{s}}}{\alpha_{d_{s}}}
$$

Então (6.85) torna-se

$$
\begin{aligned}
& E\left\{\prod_{x_{1}, \ldots, x_{d_{1}-1}} \exp \left\{-c^{\prime}\left(\lambda \prod_{j=1}^{d_{1}-1} c_{j}^{(n)}\right)^{\frac{\alpha_{d_{2}}}{1 \pm \eta_{d_{2}}}} \sum_{x_{d_{1}}}\left(\hat{\gamma}_{d_{1}}\left(\left.x\right|_{d_{1}}\right)\right)^{1 \pm \eta_{d_{1}} \frac{\alpha_{d_{2}}}{1 \pm \eta_{d_{2}}}} b^{\prime}\right\}\right\} \\
& =\exp \left\{-c^{\prime \prime} \lambda^{\frac{\alpha_{d_{1}}}{1 \pm \eta_{d_{1}}}} \prod_{j=1}^{d_{1}-1}\left(c_{j}^{(n)}\right)^{\frac{\alpha_{d_{1}}}{1 \pm \eta_{d_{1}}}} M_{j}^{(n)}\left(b^{\prime}\right)^{\frac{\alpha_{1}}{1 \pm \eta_{1}} \frac{1 \pm \eta_{2}}{\alpha_{2}}}\right\},
\end{aligned}
$$

onde $c^{\prime \prime}>0$ é uma constante. Observe agora que o produto no expoente, desconsiderando o termo $c^{\prime \prime} \lambda^{\frac{\alpha_{d_{1}}}{1 \pm \eta_{d_{1}}}}$, é

$$
b^{\prime \prime}=\left(\prod_{j=d_{p+1}+1}^{k} c_{j}^{(n)} M_{j}^{(n)}\right)^{\frac{\alpha_{d_{1}}}{1 \pm \eta_{d_{1}}}} \prod_{s=1}^{p+1}\left(\prod_{j=d_{s-1}+1}^{d_{s}-1}\left(c_{j}^{(n)}\right)^{\frac{\alpha_{d_{s}}}{1 \pm \eta_{d_{s}}}} M_{j}^{(n)}\right)^{\frac{\alpha_{d_{1}}}{1 \pm \eta_{d}} \frac{1 \pm \eta_{d_{s}}}{\alpha_{d_{s}}}}
$$


a versão de (6.84) com $p+1$, o que completa a indução. Tornando a (6.83), pela mesmas razões explicadas no parágrafo entre (6.80) e (6.83) vemos que a expressão em (6.83) converge a 1 quando $n \rightarrow \infty$. Finalmente concluimos que (6.65) converge a 0 quase certamente quando $\delta_{1}, \ldots, \delta_{k} \rightarrow 0$ e então (6.64) está provado para $k$ em geral. Isso encerra a prova de (6.34).

Proposição 6.1 estabelece (5.3) para a representação $\hat{\gamma}$ de $\gamma$, a agora cuidamos de (5.45.5). Sua prova está contida na prova da Proposição 6.1, o que nos faz apenas esboçá-la aqui. Note que as expressões (5.4-5.5), depois de dividir os $M$ 's no denominador dentro da soma, e expandir os produtos resultantes

$$
\prod_{q=2}^{p} \hat{\gamma}_{q-1}^{(n)}\left(\left.x\right|_{q-1}\right) \text { and } \prod_{q=p+2}^{j}\left(\frac{1}{M_{q}}+\hat{\gamma}_{q-1}^{(n)}\left(\left.x\right|_{q-1}\right)\right),
$$

ambos podem ser escritos como a soma sobre uma número finito de termos da forma

$$
\sum_{x_{1}} \breve{\gamma}_{1}^{(n)}\left(x_{1}\right) \ldots \sum_{x_{l}} \breve{\gamma}_{l}^{(n)}\left(\left.x\right|_{l}\right)
$$

onde $1 \leq l \leq k$ e $\breve{\gamma}_{j}^{(n)}\left(\left.x\right|_{j}\right)$ é ou $\hat{\gamma}_{j}^{(n)}\left(\left.x\right|_{j}\right)$ ou $1 / M_{j+1}^{(n)}=c_{j}^{(n)}$ para $j=1, \ldots, l$, e o derradeiro caso ocorre para ao menos um $j$ (quando $q=p+1$ em (5.4-5.5)). Em seguida dividimos cada soma $\sum_{x_{j}}$ em 3 partes como em (6.61), com o sobrescrito (3) para os casos nos quais $\breve{\gamma}_{j}^{(n)}\left(\left.x\right|_{j}\right)=c_{j}^{(n)}$. Procedemos então como antes para os últimos casos de (6.65) (veja parágrafo de $(6.79)$ e $(6.80))$, e a convergência quase certa a 0 ocorre.

\subsection{Limite de escala do modelo de armadilhas do GREM}

Após conferirmos que as condições (5.2-5.5) valem quase certamente para a representação $\hat{\gamma}$ de $\gamma$, enunciamos o teorema principal deste capítulo.

Teorema 6.1. Seja $X_{k}^{(n)} \sim T M_{k}\left(\mathbb{T}_{k}^{(n)}, \underline{\gamma_{k}^{(n)}}\right)$ e $X_{k} \sim K_{k}\left(\mathbb{T}_{k}, \underline{\gamma_{k}}\right)$. Então

$$
\left(\tilde{X}_{k}^{(n)}, \underline{\gamma_{k}^{(n)}}\right) \Rightarrow\left(X_{k}, \underline{\gamma_{k}}\right)
$$

onde $\Rightarrow$ significa convergência fraca no produto do espaço de Skorohod com o espaço das medidas finitas em $\mathbb{N}_{*}^{k}$.

Demonstração. Relembrando o acoplamento introduzido no início da Seção 6.4, a prova segue diretamente da Proposição 6.1 e do Teorema 5.1. 


\section{A A métrica de Skorohod}

Seguimos aqui a seção 3.5 de [10]. Seja $(E, d)$ um espaço métrico e seja $q=d \wedge 1$. As trajetórias de processos estocásticos são contínuas à direita e têm limites à esquerda. Assim, seja $D_{E}[0, \infty)$ o espaço das funções càdlàg (ou seja, $x:[0, \infty) \rightarrow E$ tal que, para cada $t \geq 0$, $\lim _{s \rightarrow t+} x(s)=x(t)$ e $\lim _{s \rightarrow t-} x(s) \equiv x(t-)$ existe).

Agora apresentamos a métrica de Skorohod metric em $D_{E}[0, \infty)$ sob a qual ele é um espaço métrico separável se $E$ é separável, e é completo se $(E, d)$ é completo. Seja $\Delta^{\prime}$ a coleção de funções estritamente crescentes e sobrejetoras $\lambda$ que mapeiam $[0, \infty)$ sobre $[0, \infty)$ (em particular, $\lambda(0)=0$, $\lim _{t \rightarrow \infty} \lambda(t)=\infty$ e $\lambda$ é contínua). Seja $\Delta$ o conjunto de funções contínuas lipschitziana $\lambda \in \Delta^{\prime}$ tais que

$$
\phi(\lambda)=\sup _{0 \leq s<t}\left|\log \frac{\lambda_{t}-\lambda_{s}}{t-s}\right|<\infty .
$$

Para $x, y \in D_{E}[0, \infty)$, defina

$$
\rho(x, y)=\inf _{\lambda \in \Lambda}\left[\phi(\lambda) \vee \int_{0}^{\infty} e^{-u} \rho(x, y, \lambda, u) d u\right],
$$

onde

$$
\rho(x, y, \lambda, u)=\sup _{t \geq 0} q(x(t \wedge u), y(\lambda(t) \wedge u)) .
$$

A topologia induzida em $D_{E}[0, \infty)$ pela métrica $\rho$ chama-se topologia de Skorohod.

Lema A.1. Se $x \in D_{E}[0, \infty)$, então $x$ tem no máximo uma quantidade enumerável de pontos de descontinuidade.

Demonstração. Veja o Lema 3.5.1 de [10].

Proposição A.1. Seja $\left\{x_{n}\right\} \subset D_{E}[0, \infty)$ e $x \in D_{E}[0, \infty)$. Então $\lim _{n \rightarrow \infty} \rho\left(x_{n}, x\right)=0$ se e só se existe $\left\{\lambda_{n}\right\} \subset \Delta$ tal que

$$
\lim _{n \rightarrow \infty} \phi\left(\lambda_{n}\right)=0 \text { e } \lim _{n \rightarrow \infty} \rho\left(x_{n}, x, \lambda_{n}, u\right)=0
$$

para todos os pontos de continuidade $u$ de $x$. Em particular, $\lim _{n \rightarrow \infty} \rho\left(x_{n}, x\right)=0$ implica que

$$
\lim _{n \rightarrow \infty} x_{n}(u)=\lim _{n \rightarrow \infty} x_{n}(u-)=x(u) \text { para todo ponto de continuidade } u \text { de } x
$$

Demonstração. Veja a Proposição 3.5.2 de [10]. 
Com o propósito de ilustrar uma aplicação de (A.2) mostramos um exemplo.

Exemplo A.1. Seja $E=\{0,1\}$ e d a métrica discreta. Dado $T>0$, considere as funções $x, x_{n}:[0, \infty) \rightarrow\{0,1\}$ tais que

$$
\begin{gathered}
x(t)=\left\{\begin{array}{ll}
0, & \text { se } t<T \\
1, & \text { se } t \geq T
\end{array},\right. \\
x_{n}(t)=\left\{\begin{array}{lll}
0, & \text { se } & t<T+1 / n \\
1, & \text { se } & t \geq T+1 / n
\end{array} .\right.
\end{gathered}
$$

Fixado $n \in \mathbb{N}_{*}$, estimemos $\rho\left(x, x_{n}\right)$. Consideramos a distorção

$$
\lambda_{n}(t)=\left\{\begin{array}{ll}
\left(1+\frac{1}{T n}\right) t, & \text { se } \quad 0 \leq t<T \\
\left(1-\frac{1}{2 n}\right) t+\frac{T+2}{2 n}, & \text { se } \quad T \leq t \leq T+2 \\
t, & \text { se } t \geq T+2
\end{array} .\right.
$$

Após dividirmos as contas em 3 partes temos

$$
\rho\left(x, x_{n}, \lambda_{n}, u\right)=\left\{\begin{array}{lll}
0, & \text { se } & 0 \leq u<T \\
1, & \text { se } & T \leq u \leq T+\frac{1}{n} \\
0, & \text { se } & u \geq T+\frac{1}{n}
\end{array}\right.
$$

então

$$
\int_{0}^{\infty} e^{-u} \rho\left(x, x_{n}, \lambda_{n}, u\right)=e^{-T}-e^{-T+\frac{1}{n}}
$$

Em seguida, olhando para (A.1) temos $\phi\left(\lambda_{n}\right)=\log \left(1+\frac{1}{T n}\right)$. Assim, com (A.2) em mente obtemos

$$
\rho\left(x, x_{n}\right) \leq \log \left(1+\frac{1}{T n}\right) \vee\left[e^{-T}-e^{-T+\frac{1}{n}}\right] .
$$

Agora observe que ambos os termos no membro direito de (A.5) vão a 0 quando $n \rightarrow \infty$ e portanto $\rho\left(x, x_{n}\right) \rightarrow 0$. Se tivéssemos lidado com a métrica do supremo, que é dada por $m_{s}(x, y)=\sup _{t \geq 0} d(x(t), y(t))$ para todo $x, y \in D_{E}[0, \infty)$, aqui teríamos $m_{s}\left(x, x_{n}\right)=1$ para todo $n \in \mathbb{N}_{*}$. 


\section{B Processos Pontuais}

Enunciamos os principais conceitos e teoremas relacionados a processos pontuais que são usados na tese. Nossa abordagem aqui é breve e nós não mostramos a prova dos resultados. Sugerimos ao leitor consultar [19] para mais detalhes. Começamos apresentando a definição de medidas pontuais e processos pontuais e, em seguida, nos voltamos para processos pontuais de Poisson.

O espaço de estados onde os pontos vivem será um espaço métrico denotado por $E$, que para nossos propósitos será $\mathbb{R}^{d}$. Seja $\mathcal{E}$ a $\sigma$-álgebra de Borel dos subcnjuntos de $E$. Para $\gamma \in E$, defina a medida $\mathbb{1}_{\gamma}$ em $A \in \mathcal{E}$ por

$$
\mathbb{1}_{\gamma}(A)=\left\{\begin{array}{lll}
1 & \text { se } & \gamma \in A \\
0 & \text { se } & \gamma \notin A
\end{array}\right.
$$

Seja $\left\{\gamma_{i}, i \geq 1\right\}$ uma coleção enumerável de pontos de $E$. Então uma medida pontual $m$ em $E$ é definida por

$$
m \equiv \sum_{i=1}^{\infty} \mathbb{1}_{\gamma_{i}}
$$

e se $K \in \mathcal{E}$ é compacto então $m(K)<\infty$.

Chame $M_{p}(E)$ o espaço de todas medidas pontuais definidas em $E$ e defina uma $\sigma$-álgebra $\mathcal{M}_{p}(E)$ de subconjuntos de $M_{p}(E)$ como a menor $\sigma$-álgebra contendo todos os conjuntos da forma $\left\{m \in M_{p}(0, \infty) ; m(F) \in B\right\}$ para $F \in E, B \in \mathcal{B}([0, \infty)]$.

Um processo pontual em $E$ é uma aplicação mensurável $N$ de um espaço de probabilidade $(\Omega, \mathcal{F}, P) \rightarrow\left(M_{p}(E), \mathcal{M}_{p}(E)\right)$, isto é, para cada $w \in \Omega N(w)$ é uma medida pontual em $E$ e $N(w, F)$ é o número de pontos em $F$ para a realização $w$. Denotamos por $P_{N}$ a lei de probabilidade do processo pontual $N$ em $\mathcal{M}_{p}(E)$, ou seja, $P_{N}=P \circ N^{-1}=P(N \in \cdot)$. Agora apresentamos um critério mais intuitivo para afirmar que $N$ é um processo pontual.

Proposição B.1. N é um processo pontual se e só se a aplicação $w \rightarrow N(w, F)$ é mensurável de $(\Omega, \mathcal{F}) \rightarrow([0, \infty], \mathcal{B}([0, \infty)])$ para cada $F \in \mathcal{E}$.

Demonstração. Veja Proposição 3.1 de [19].

A medida de intensidae $\mu$ de um processo pontual $N$ é uma is uma medida definida por

$$
\mu(F)=E(N(F))=\int_{\Omega} N(w, F) P(d w)=\int_{M_{p}(E)} m(F) P_{N}(d m)
$$


Uma ferramenta importante usada para estudar a convergência fraca de processos pontuais em $\left(M_{p}(E), \mathcal{M}_{p}(E)\right)$ é a transformada de Laplace. Seja $f: E \rightarrow[0, \infty)$ uma função mensurável e defina

$$
N(w, f)=\int_{E} f(x) N(w, d x)
$$

$N(f)$ é uma variável aleatória. Então o funcional de Laplace de $N$ é a transformada de Laplace da lei de $N$ :

$$
\begin{aligned}
\Psi_{N}(f) \equiv E \exp \{-N(f)\} & =\int_{\Omega} \exp \{-N(w, f)\} P(d w) \\
& =\int_{M_{p}(E)} \exp \left\{-\int_{E} f(x) m(d x)\right\} P_{N}(d m) .
\end{aligned}
$$

Proposição B.2. O funcional de Laplace $\Psi_{N}$ de $N$ determina unicamente a lei de $N$.

Demonstração. Veja a Proposição 3.5 de [19].

Com estas ideias em mente agora podemos lidar com processos pontuais de Poisson.

Dada uma medida localmente finita $\mu$ em $\mathcal{E}$, um processo pontual $N$ é chamdo um processo pontual de Poisson com medida de intensidade $\mu$ (brevemente, $N$ é $\operatorname{PPP}(\mu)$ )se $N$ satisfaz

a) para qualquer $F \in \mathcal{E}$ e $k \in \mathbb{N}$

$$
P(N(F)=k)=\left\{\begin{array}{ccc}
\exp (\mu(F)) \frac{\mu(F)^{k}}{k !} & \text { se } & \mu(F)<\infty \\
0 & \text { se } & \mu(F)=\infty
\end{array}\right.
$$

b) para qualquer $k \geq 1$, se $F_{1}, \ldots, F_{k}$ são conjuntos mutualmente disjuntos em $\mathcal{E}$ então $N\left(F_{i}\right), i=1, \ldots, k$ são variáveis aleatórias independentes.

Proposição B.3. $\quad$ i) $P P P(\mu)$ existe. Sua lei é unicamente determinada por a) e b) na definição prévia.

ii) o funcional de Laplace de $P P P(\mu)$ para $f \geq 0$ mensurável é dado por

$$
\Psi_{N}(f)=\exp \left\{-\int_{E}\left(1-e^{-f(x)}\right) \mu(d x)\right\}
$$

e inversamente um processo pontual com funcional de Laplace da forma (B.7) deve ser $\operatorname{PPP}(\mu)$. 
Demonstração. Veja a Proposição 3.6 de [19].

Proposição B.4. Sejam $E_{i}, i=1,2$ dois espaços métricos localmente compactos com base enumerável. Sejam $\mathcal{E}_{i}, i=1,2$ as $\sigma$-álgebras associadas. Seja $T:\left(E_{1}, \mathcal{E}_{1}\right) \rightarrow\left(E_{2}, \mathcal{E}_{2}\right)$ mensurável. Se $N$ é $P P P(\mu)$ em $E_{1}$ então

$$
\hat{N}:=N \circ T^{-1} \quad \text { é } P P P\left(\hat{\mu}=\mu \circ T^{-1}\right) \text { em } E_{2} .
$$

Se temos uma representação

$$
N=\sum_{i} \mathbb{1}_{X_{i}}
$$

então

$$
\hat{N}=N \circ T^{-1}=\sum_{i} \mathbb{1}_{T X_{i}}
$$

Demonstração. Veja a Proposição 3.7 de [19].

Teorema B.1 (Teorema de Campbell). Seja $N$ um processo de Poisson em E com medida de intensidade $\mu$, seja $f: E \rightarrow \mathbb{R}$ mensurável. Então a soma

$$
\Sigma=\sum_{i=1}^{\infty} f\left(X_{i}\right)
$$

é absolutamente convergente com probabilidade 1 se e só se

$$
\int_{E} \min (|f(x)|, 1) \mu(d x)<\infty
$$

Se essa condição vale, então

$$
\mathbb{E}\left(e^{\theta \Sigma}\right)=\exp \left\{\int_{E}\left(e^{\theta f(x)}-1\right) \mu(d x)\right\}
$$

para qualquer complexo $\theta$ para o qual a integral à direita converge e, em particular, sempre que $\theta$ é puramente imaginário. Ademais

$$
\mathbb{E}(\Sigma)=\int_{E} f(x) \mu(d x)
$$

no sentido de que a esperana existe se e só se a integral converge, e eles então são iguais. Se (B.14) converge, então

$$
\operatorname{var}(\Sigma)=\int_{E} f(x)^{2} \mu(d x),
$$

finito ou infinito. 
Demonstração. Veja a seção 3.2 de [16].

Para discutir a convergência fraca de processos pontuais precisamos de uma noção de convergência em $M_{p}(E)$. Seja $\rho$ uma métrica em $E$ que torna $E$ um espạ métrico completo e separável.

Seja $C_{K}(E)$ as funções reais contínuas em $E$ com suporte compacto. $C_{K}^{+}(E)$ é o subconjunto de $C_{K}(E)$ que consiste das funções não-negativas. Sejam $M_{+}(E)$ todas as medidas não-negativas localmente finitas em $(E, \mathcal{E})$ e defina $\mathcal{M}_{+}(E)$ como a menor $\sigma$-álgebra de subconjuntos de $M_{+}(E)$ que faz as aplicações $m \rightarrow m(f)=\int_{E} f d m$ de $M_{+}(E) \rightarrow \mathbb{R}$ mensuráveis para toda $f \in C_{K}^{+}(E)$.

Para $\mu_{n}, \mu \in M_{+}(E)$ dizemos que $\mu_{n}$ converge vaguamente a $\mu$ e escrevemos $\mu_{n} \stackrel{v}{\rightarrow} \mu$ se $\mu_{n}(f) \rightarrow \mu(f)$ para todo $f \in C_{K}^{+}(E)$, ou seja,

$$
\int_{E} f(x) \mu_{n}(d x) \rightarrow \int_{E} f(x) \mu(d x) .
$$

Proposição B.5. Sejam $\mu, \mu_{1}, \mu_{2}, \ldots$ em $M_{+}(E)$. São equivalentes:

i) $\mu_{n} \stackrel{v}{\rightarrow} \mu$

ii) $\mu_{n}(B) \rightarrow \mu(B)$ para todo $B$ relativamente compacto para o qual $\mu(\partial B)=0$, isto é, a fronteira de $B$ tem $\mu$ medida 0.

iii) $\limsup _{n \rightarrow \infty} \mu_{n}(K) \leq \mu(K)$ e $\liminf _{n \rightarrow \infty} \mu_{n}(G) \geq \mu(G)$ para todo compacto $K$ e todo aberto, relativamente compacto $G$.

Demonstração. Veja a Proposição 3.12 de [19].

A convergência vaga de medidas pontuais $\mu_{n} \rightarrow \mu$ tem a seguinte interpretação em termos de convergência dos pontos de $\mu_{n}$ para os pontos de $\mu$.

Proposição B.6. Suponha $\mu_{n}, \mu \in M_{p}(E)$ e $\mu_{n} \stackrel{v}{\rightarrow} \mu$. Para $K$ compacto e satisfazendo $\mu(\partial K)=0$ temos para $n \geq n_{K}$ uma rotulação dos pontos de $\mu_{n}$ e $\mu$ tal que

$$
\mu_{n}(\cdot \cap K)=\sum_{i=1}^{p} \mathbb{1}_{\gamma_{i}^{(n)}}, \quad \mu(\cdot \cap K)=\sum_{i=1}^{p} \mathbb{1}_{\gamma_{i}}
$$

e em $E^{p}$

$$
\left(\gamma_{i}^{(n)}, 1 \leq i \leq p\right) \rightarrow\left(\gamma_{i}, 1 \leq i \leq p\right) \quad \text { quando } n \rightarrow \infty
$$

(Claramente, $E^{p}$ tem a topologia produto de modo que a convergência de vetores em $E^{p}$ significa convergência componente a componente.) 
Dizemos que uma sequência de processos pontuais $N_{n}$ converge fracamente a um processo pontual $N$ (escrito $N_{n} \Rightarrow N$ ) se para toda função limitada e contínua $f$ em $M_{p}(E)$

$$
\int_{M_{p}(E)} f(x) P_{n}(d x) \rightarrow \int_{M_{p}(E)} f(x) P(d x)
$$

Proposição B.7. Se $\xi_{n}, n \geq 0$ são medidas aleatórias, ou seja, elementos aleatórios em $M_{+}(E)$, então $\xi_{n} \Rightarrow \xi$ se e só se $\Psi_{\xi_{n}}(f) \rightarrow \Psi_{\xi}(f)$ para toda $f \in C_{K}^{+}(E)$.

Demonstração. Veja a Proposição 3.19 de [19].

Proposição B.8. Para cada $n$ suponha $\left\{X_{n, j}, j \geq 1\right\}$ são elementos aleatórios i.i.d. de $(E, \mathcal{E})$ e $\mu$ é uma medida localmente finita em $(E, \mathcal{E})$. Defina

$\xi_{n}=\sum_{j=1}^{\infty} \mathbb{1}_{\left(j n^{-1}, X_{n, j}\right)}$ e suponha $\xi$ é PPP em $[0, \infty) \times E$ com medida média $d t \times d \mu$. Então $\xi_{n} \Rightarrow \xi$ em $M_{p}([0, \infty) \times E)$ se e só se

$$
n P\left(X_{n, 1} \in \cdot\right) \stackrel{v}{\rightarrow} \mu \text { em } E
$$

Demonstração. Veja a Proposição 3.21 de [19].

\section{Variação Regular}

Definição C.1. Uma função mensurável $U:(0, \infty) \rightarrow(0, \infty)$ é de variação regular em $\infty$ com indice $\alpha \in \mathbb{R}$ (escrito $U \in R_{\alpha}$ ) se

$$
\lim _{t \rightarrow \infty} \frac{U(a t)}{U(t)}=a^{\alpha} .
$$

Chamamos $\alpha$ o expoente de variação. Com mudanças óbvias podemos conversar sobre variação regular em $0+$, isto é, $U(t)$ é de variação regular em $\infty$ se e só se $U(1 / t)$ é variação regular em 0+. Quando $\alpha=0$ dizemos que a função é de variação lenta. De fato, cada função $U \in R_{\alpha}$ pode ser representada como $U(t)=L(t) t^{\alpha}$, onde $L$ é uma função de variação lenta.

Teorema C.1. L é de variação lenta em $\infty$ se e só se L pode ser representado como

$$
L(x)=\kappa(x) \exp \left\{\int_{1}^{x} \frac{\epsilon(y)}{y} d y\right\},
$$

para $x>0$, onde $\kappa:(0, \infty) \rightarrow(0, \infty), \epsilon:(0, \infty) \rightarrow(0, \infty)$ e $\kappa(x) \rightarrow \kappa>0, \epsilon(x) \rightarrow 0$ quando $x \rightarrow \infty$. 
Demonstração. Veja o Corolário do Teorema 0.6 de [19].

Lema C.1. Se L é de variação lenta no infinito então

$$
x^{-\varepsilon}<L(x)<x^{\varepsilon}
$$

para qualquer $\varepsilon>0$ fixado e todo $x$ suficientemente grande.

Demonstração. Veja o Lema VIII.8.2 de [11].

Lema C.2. Se $f \in R_{\rho}$ com $\rho<0$, existe $g \in R_{1 / \rho}(0+)$ com

$$
f(g(x)) \sim g(f(x)) \sim x, x \rightarrow 0 .
$$

Aqui g (uma inversa assintótica' de f) é determinada dentro da equivalência assintótica, e uma versão de $g$ é $f^{-1}$.

Demonstração. Veja o Teorema 1.5.12 de [1].

\section{Variáveis aleatórias estáveis}

Ao longo desta seção $X, X_{1}, X_{2}, \ldots$ denota variáveis aleatórias não-degeneradas i.i.d. e $S_{n}=$ $X_{1}+\ldots+X_{n}$. Existem algumas definições equivalentes de variáveis alaetórias estáveis e aqui duas delas são enunciadas.

Definição D.1. Uma variável aleatória $X$ é estável (em sentido amplo) se para cada $n$ existem constantes $c_{n}>0, b_{n}$ tais que

$$
S_{n}=c_{n} X+b_{n}
$$

$X$ é estável em sentido estrito se (D.1) vale com $b_{n}=0$.

Definição D.2. Uma variável aleatória $X$ tem distribuição estável se existem parâmetros $0<\alpha \leq 2, \sigma \geq 0,-1 \leq \beta \leq 1$ e $\mu \in \mathbb{R}$ tais que sua função característica tem a forma que se segue:

$$
E(\exp (i \theta X))= \begin{cases}\exp \left\{-\sigma^{\alpha}|\theta|^{\alpha}\left[1-i \beta(\operatorname{sign}(\theta)) \tan \left(\frac{\pi \alpha}{2}\right)\right]+i \mu \theta\right\} & \text { se } \alpha \neq 1 \\ \exp \left\{-\sigma|\theta|\left[1+i \beta \frac{2}{\pi}(\operatorname{sign}(\theta)) \ln |\theta|\right]+i \mu \theta\right\} & \text { se } \alpha=1\end{cases}
$$

Notação: $X \sim S_{\alpha}(\sigma, \beta, \mu)$. O parâmetro $\alpha$ é o índice de estabilidade $e$

$$
\operatorname{sign}(\theta)= \begin{cases}1 & \text { se } \theta>0 \\ 0 & \text { se } \theta=0 \\ -1 & \text { se } \theta<0\end{cases}
$$


Proposição D.1. A transformada de Laplace da variável aleatória $X \sim S_{\alpha}(\sigma, 1,0), 0<$ $\alpha \leq 2, \sigma>0$, é igual a

$$
E(\exp (\lambda X))= \begin{cases}\exp \left\{-\frac{\sigma^{\alpha}}{\cos \left(\frac{\pi \alpha}{2}\right)} \lambda^{\alpha}\right\} & \text { se } \alpha \neq 1 \\ \exp \left\{-\frac{\sigma 2}{\pi} \lambda \ln \lambda\right\} & \text { se } \alpha=1\end{cases}
$$

Demonstração. Veja a Proposição 1.2 .12 de [20].

Para a próxima definição, seja $\left\{X_{n}\right\}_{n \geq 1}$ uma sequência i.i.d. de variáveis alaetórias com distribuição comum $F$. Seja $M_{n}=\bigvee_{i=1}^{n} X_{i}$. A distribuição de $M_{n}$ é dada por $F^{n}=(F)^{n}$.

Definição D.3. Dizemos que uma distribuição F está na bacia de atração de G se existem $a_{n}>0$ e $b_{n}$ tais que

$$
F^{n}\left(a_{n} t+b_{n}\right) \rightarrow G(t) \quad \text { fracamente. }
$$

G é uma distribuição de valor extremo.

Teorema D.1. Suponha que $X_{1}, X_{2}, \ldots$ são v.a. com uma distribuição que satisfaz

i) $\lim _{t \rightarrow \infty} P\left(X_{1}>t\right) / P\left(\left|X_{1}\right|>t\right)=\theta \in[0,1]$

ii) $P\left(X_{1}>t\right)=t^{-\alpha} L(t)$,

onde $\alpha<2$ e L é de variação lenta em $\infty$. Seja $S_{n}=X_{1}+\ldots+X_{n}, a_{n}=\inf \{t \geq 0$ : $\left.P\left(\left|X_{1}\right|>t\right) \leq n^{-1}\right\}$ e $b_{n}=n E\left(X_{1} \mathbb{1}_{\left(\left|X_{1}\right| \leq a_{n}\right)}\right)$, então

$$
\frac{S_{n}-b_{n}}{a_{n}} \Rightarrow Y
$$

onde $Y$ tem uma distribuição não-degenerada.

Demonstração. Veja o Teorema 3.7.2 de [9].

A fim de justicarmos a escolha de variáveis aleatórias no primeiro parágrafo da Seção 6.1, mostremos que $\operatorname{com} \theta=1$ (isto é, v.a. positiva) e $b_{n}=0$ em Teorema D.1 a convergência em (D.3) vale. Por (6.21), com $n$ e $a_{n}$ em vez de $M_{j}^{(n)}$ e $M_{j+1}^{(n)}$, respectivamente, obtemos 
$n P\left(X_{1}>a_{n}\right) \rightarrow 1$ quando $n \rightarrow \infty$. Por sua vez, por (C.1) o quociente $\frac{P\left(X_{1}>a_{n} t\right)}{P\left(X_{1}>a_{n}\right)} \rightarrow t^{-\alpha}$ quando $n \rightarrow \infty$. Então, para $t>0$,

$$
\begin{aligned}
F^{n}\left(a_{n} t\right)=P\left(M_{n} / a_{n} \leq t\right) & =\left(1-\frac{P\left(X_{1}>a_{n} t\right)}{P\left(X_{1}>a_{n}\right)} \frac{n P\left(X_{1}>a_{n}\right)}{n}\right)^{n} \\
& \rightarrow \exp \left(-t^{-\alpha}\right)
\end{aligned}
$$

que é chamada de distribuição de Fréchet com parâmetro $\alpha$.

Teorema D.2. Y é o limite de $\left(X_{1}+\ldots+X_{n}-b_{n}\right) / a_{n}$ para alguma sequência $X_{i}$ se e só se $Y$ tem lei estável.

Demonstração. Veja o Teorema 3.7.4 de [9]. 


\section{Referências}

[1] Bingham, N.; Goldie, C.; Teugels, J. (1987) Regular Variation, Encyclopedia of mathematics and its applications, vol. 27, Cambridge University Press, Cambridge.

[2] Bolthausen, E.; Sznitman, A. S. (1998) On Ruelle's probability cascades and an abstract cavity method, Comm. Math. Physics 197, 247-276.

[3] Ben Arous, G.; Černý, J. (2006) Dynamics of trap models, in: Mathematical statistical physics vol. LXXXIII of Les Houches summer school proceedings.

[4] Ben Arous, G.; Bovier, A. Černý, J. (2008) Universality of random energy model-like ageing in mean field spin glasses, J. Stat. Mech. Theory Exp. 4, 4003-4011.

[5] Bouchaud, J. -P. (1992) Weak ergodicity breaking and aging in disordered systems, $J$. Phys. I (France) 2, 1705-1713.

[6] Chung, K. L. (1967) Markov chains with stationary transition probabilities, SpringerVerlag.

[7] Derrida, B. (1981) Random energy model: an exactly solvable model of disordered systems, Phys. Rev. B 24, no. 5, 2613-2626.

[8] Derrida, B. (1985) A generalisation of the random energy model that includes correlations betweens the energies, J. Phys. Lett. 46, 401-40\%.

[9] Durrett, R. (2010) Probability: Theory and Examples, Duxbury Press.

[10] Ethier, S. N.; Kurtz, T. G. (1986) Markov Processes. Characterization and Convergence, Wiley.

[11] Feller, W. (1971) An introduction to the probability theory and its applications, vol.2, Wiley.

[12] Fontes, L. R. G.; Mathieu, P. (2008) K-processes, scaling limit and aging for the trap model on the complete graph, Annals of Probability 36, 1322-1358.

[13] Fontes, L. R. G.; Isopi, M.; Newman, C. M. (2002) Random walks with strongly inhomogeneous rates and singular diffusions: convergence, localization and aging in one dimension. Annals of Probability 30, 579-604. 
[14] Gayrard, V. (2010) Aging in reversible dynamics of disordered systems I. Emergence of the arcsine law in Bouchaud's asymetric trap model on the complete graph (preprint).

[15] Grimmett, G.; Stirzaker, D. C. (2001) Probability and Random Processes, Oxford.

[16] Kingman, J. F. C. (1987) Poisson Processes, Oxford.

[17] Lepage, R.; Woodroofe, M.; Zinn, J. (1981) Convergence to a stable distribution via order statistics. Annals of Probability 9, no. 4, 624-632.

[18] Peixoto, G. R. C. (2011) Um estudo sobre o processo K não homogêneo. Master thesis, Instituto de Matemática e Estatística, Universidade de São Paulo.

[19] Resnick, S. I. (1987) Extreme values, regular variation, and point processes, Springer.

[20] Samorodnitsky, G.; Taqqu, M. S. (1994) Stable non-gaussian random processes, Chapman and Hall. 